NBER WORKING PAPER SERIES

\title{
HETEROGENEOUS HOUSEHOLDS UNDER UNCERTAINTY
}

\author{
Pietro Veronesi \\ Working Paper 25448 \\ http://www.nber.org/papers/w25448 \\ NATIONAL BUREAU OF ECONOMIC RESEARCH \\ 1050 Massachusetts Avenue \\ Cambridge, MA 02138 \\ January 2019
}

I thank John Heaton and Lubos Pastor for their comments. I acknowledge financial support from the Fama-Miller Center for Research in Finance and by the Center for Research in Security Prices at the University of Chicago Booth School of Business. The views expressed herein are those of the author and do not necessarily reflect the views of the National Bureau of Economic Research.

NBER working papers are circulated for discussion and comment purposes. They have not been peer-reviewed or been subject to the review by the NBER Board of Directors that accompanies official NBER publications.

(C) 2019 by Pietro Veronesi. All rights reserved. Short sections of text, not to exceed two paragraphs, may be quoted without explicit permission provided that full credit, including (C) notice, is given to the source. 
Heterogeneous Households under Uncertainty

Pietro Veronesi

NBER Working Paper No. 25448

January 2019

JEL No. E21,G1,G11,G12

\begin{abstract}
$\underline{\text { ABSTRACT }}$
I characterize a dynamic economy under general distributions of households' risk tolerance, endowments, and beliefs about long-term growth. As the economy expands and the stock market rises (a) the fraction of households with declining consumption-share increases; (b) the wealthshare of high risk-tolerant households increases; (c) richer households' wealth display a higher CAPM beta; and (d) households' portfolios change qualitatively. A log-utility investor for instance borrows in contractions but lends in expansions. Variations in uncertainty and expected growth generate trading volume due to risk sharing. Higher uncertainty increases stock prices, risk premiums, volatility, wealth inequality and the dispersion of portfolio holdings, consistently with the events in the late 1990s.
\end{abstract}

Pietro Veronesi

University of Chicago

Booth School of Business

5807 South Woodlawn Avenue

Chicago, IL 60637

and NBER

pietro.veronesi@chicagobooth.edu 


\section{Introduction}

Empirical studies on households' risk preferences using different estimation strategies and different samples agree on one fact: The distribution of risk tolerance is wide, with some households extremely risk averse and with others close to risk-neutrality. ${ }^{1}$ In a world with large and time varying macroeconomic uncertainty, such large differences in risk-preferences must impact agents' trading behavior and wealth dynamics. For instance, it is well known that the late 1990s were characterized by rising price valuation, volatility, and trading volume. Wealth inequality, moreover, also trended up strongly in that period. ${ }^{2}$ Raising macroeconomic uncertainty with heterogeneous preferences predict just that.

Specifically, I embed general risk-preference distributions in a dynamic model of asset pricing with macroeconomic uncertainty and obtain numerous predictions. I first focus on a benchmark model without learning to obtain benchmark results: In this setting, higher dispersion of risk preferences generates higher Sharpe ratios and risk-free rates in bad times but lower ones in good times. As the economy expands and the stock market rises (a) the fraction of households whose consumption-share declines with output increases; (b) the wealth-share of high risk-tolerant households increases; (c) richer households' net worth display a higher CAPM beta; and (d) households' portfolio allocations change qualitatively. A log-utility investor, for instance, borrows in bad times but lends in good times. Points (a), (b), (c) are consistent with recent trends (See Saez and Zucman (2016), Gomez (2018)). ${ }^{3}$ The key driver of these results is risk sharing, as households with higher risk tolerance insure those with lower risk tolerance by selling them risk-free bonds and taking aggressive positions in stocks. They thus gain the most as the economy expands.

The extension to uncertainty about long-term growth and learning yields additional novel results. First, higher uncertainty entails additional motives for risk-sharing and thus households with higher risk tolerance take on even more extreme positions in stocks than those with lower risk tolerance. In particular, an endogenous increase in uncertainty generates higher dispersion in portfolio allocations across households which is achieved through higher trading volume, as more risk tolerant households buy stocks from the less risk tolerant ones. I show that an endogenous increase in uncertainty thus increases trading volume as well as stock valuations, return volatility, and wealth inequality.

\footnotetext{
${ }^{1}$ See e.g. Barsky, Juster, Kimball and Shapiro (1997), Kimball et al. (2008, Figure 1), Chiappori et al. (2014, Figure 2).

${ }^{2}$ See e.g. Saez and Zucman (2016).

${ }^{3}$ In a related model with multiple countries and inequality-averse households, Pastor and Veronesi (2018) show that globalization exacerbates the inequality due to risk sharing highlighted in (a) and (b) and thus lead households to vote for a populist who promises to end globalization.
} 
These results provide theoretical foundation to the events of the late 1990s that saw record breaking valuations, high volatility, trading volume, and dispersion of portfolio holdings across managers (see e.g. Greenwood and Nagel (2009)). The existence of large trading volume around "bubble" events has been taken as evidence of behavioral biases (see e.g. Scheinkman (2014) for a review), but increasing economic uncertainty in the spirit of Pastor and Veronesi (2006) combined with heterogeneous preferences as in this paper provide a plausible alternative story. Moreover, rising uncertainty also increases the wealth dispersion across agents, thereby explaining the large increase in wealth inequality in the late 1990s (see e.g. Saez and Zucman (2016)).

Under uncertainty and learning, households' portfolio allocations become extreme. Indeed, households with low risk tolerance may even short stocks to hedge against bad news about long-term economic growth. Negative shocks to aggregate output signal even lower output going forward and such households can hedge against this bad news by shorting stocks and buying bonds. Indeed, their wealth is negatively correlated with the aggregate economy. Time varying expected growth and uncertainty are thus potentially important channels for trading dynamics in a learning environment.

The focus on continuous distributions of risk tolerance also highlights the behavior of households with intermediate risk tolerance which are not visible in standard two-agents models. For instance, as the economy expands agents with intermediate risk tolerance are first borrowers and then lenders, with the intensity of borrowing and lending determined by their risk tolerance. This behavior is due to the change in risk-return characteristics of risky stocks and interest rates in equilibrium. As the economy expands, the aggregate Sharpe ratio declines, decreasing the attractiveness of stock investment. Although also the risk-free rate declines, the former effect dominates and induce intermediate agents to move out from stocks and buy bonds.

My paper is related to numerous strands of literature: The first and more obvious is the traditional literature on heterogeneous risk preferences, risk sharing, and asset prices. Differently from the classic papers of Dumas (1989), Wang (1996), Longstaff and Wang (2013), and Bahmra and Uppal (2014), I consider a continuum of agents and obtain relations about the whole distribution of wealth, and not only of two types. This is important to discuss issues related to wealth distribution, for instance, or the behavior of households in the middle of the distribution, which cannot be studied in two-agents models. Finally, I focus on the impact of learning dynamics on portfolio holdings and asset prices, which is mostly not discussed in this earlier research. 
Cvitanic et al. (2011) consider $N$ types of heterogeneous agents and obtain several results that are related to some in this paper. While Cvitanic et al. consider multiple sources of heterogeneity (preferences, beliefs, discounting), I rather focus on generic distributions of risk-tolerance and endowments and learning about long-term growth. Unlike that paper, I show that heterogeneity and uncertainty explain high valuation, trading volume, and volatility, for instance, among other results. The papers are complementary from a technical standpoint, but they focus on different issues.

Chan and Kogan (2002), Xiorius and Zapatero (2007) and Santos and Veronesi (2018) also consider models with a continuum of agents, but under the assumption of habit formation or time-varying risk preferences. My focus is instead on the impact of uncertainty and learning on households' consumption and trading patterns, and their relation to asset prices, when households have standard preferences with constant relative risk aversion (CRRA). From a technical standpoint, I exploit similar methodologies as in Chan and Kogan (2002) and Xiorius and Zapatero (2007).

Pastor and Veronesi (2018) study a two-country model that exploits heterogeneous risk preference within country and across countries. Unlike this paper, that paper assumes that agents are averse to inequality, especially the consumption of the "elites". As the global economy expands, international risk sharing exacerbates inequality in the rich country. After a long expansion, the majority of the citizens in the rich country would prefer to consume less but live in a more equal society by limiting the consumption of the ultra rich. They do so by voting for a populist who promises to end globalization, as the move to autarky affects the consumption of the ultra rich the most.

My paper is also related to the literature on parameter uncertainty and learning (e.g. David (1997), Veronesi (1999, 2000), Brennan and Xia (2001), Pastor and Veronesi (2006), Ai (2010), David and Veronesi (2013), Collin-Dufressne et al. (2016) ). This literature focuses on representative agent's models and thus does not explore the implications of risk sharing under uncertainty and learning, which this paper instead develops.

Finally, my paper is related to the literature on "bubbles" and the trading patterns that are implied by such frenzy. For instance, Scheinkman and Xiong (2003) show that overconfidence and short-sale constraints make optimistic investors value stocks more than their true fundamental value thanks to a "resale option" component. Important, trading volume is related to such resale option and the existing literature emphasizes trading volume as a distinguishing pattern of behavioral biases (see e.g. Scheinkman 2014). I show that raising uncertainty with heterogeneous preferences also induces high valuation, stock volatility 
and higher dispersion in portfolio allocations, which is in line with the evidence in the late 1990s. Pastor and Veronesi (2009) show that "bubble-like" patterns in both stock prices and volatility may be due to learning about new technologies, and the "bubble" bursts due to an increase in discount rates when a new technology is adopted. That paper though does not consider heterogeneity across agents, which is the focus of this paper.

The paper develops as follows: Next section introduces the benchmark model without learning, and the implications for agents' consumption, wealth, trading, and asset prices. Section 3. discusses the the implication of uncertainty about long-term growth for risk sharing, portfolio allocation, and trading. In section 4 . I calibrate the model. I also put forwards an assumption on initial endowments that allow for a simple solution to the equilibrium fixed point problem and thus allows me to obtain simple numerical solutions for the calibration. Section 5. concludes.

\section{Benchmark Model}

Time is continuous and it spans the period $[0, T]$, where $T$ is finite but large. A finite $T$ helps deal with the non-stationary nature of the economy. There is a continuum of infinitesimal agents. All agents have preferences exhibiting Constant Relative Risk Aversion (CRRA) over their own consumption $C_{i t}$ :

$$
U_{i}\left(C_{i t}, t\right)=e^{-\phi t} \frac{C_{i t}^{1-\frac{1}{\rho_{i}}}}{1-\frac{1}{\rho_{i}}}
$$

where $\rho_{i} \neq 1$ is agent $i$ 's risk tolerance. If $\rho_{i}=1$ then $U_{i}\left(C_{i t}, t\right)=e^{-\phi t} \log \left(C_{i t}\right)$. Risk tolerance is bounded in the population, that is, $\rho_{i} \in\left(\rho_{L}, \rho_{H}\right)$, but I make no additional assumptions otherwise.

Agents can invest in a productive risky opportunity or park their savings in a risk-free bond. At time 0 , agents are born with $s_{i}$ shares of the risky asset, with $\int s_{i} d i=1{ }^{4}$ I denote $f(\rho, s)$ the joint density on $\rho_{i}$ and $s_{i}$. The risky asset has a unit price $P_{t}$ and pays a dividend $D_{t}$, where $\delta_{t}=\log \left(D_{t}\right)$ follows the process

$$
d \delta_{t}=\mu d t+\sigma d Z_{t}
$$

$Z_{t}$ is a standard Brownian motion, and $\mu$ and $\sigma$ are constants, although this restriction is

\footnotetext{
${ }^{4}$ I often use the notation $\int d i$ to indicate the integral across agents using the relevant density on $i$, without specifying the latter to avoid notational clutter. I make the density explicit when it is necessary for economic interpretation or clarity.
} 
not necessary for the results. In this section I assume $\mu$ is known to investors. Section 3. examines the case in which $\mu$ is not known but investors learn its value over time.

In addition to the risky asset, agents can borrow or lend at the risk-free rate $r_{t}$, whose value is determined in equilibrium. $B_{t}$ is the price of a risk-free bond at $t$. Both prices $P_{t}$ and $r_{t}$ are determined in equilibrium. Markets are thus dynamically complete.

Denote each agent $i$ 's position in stock $N_{i t}$ and in bonds $N_{i t}^{0}$. Each agent maximizes the intertemporal utility

$$
\max _{\left\{C_{i t}, N_{i t}, N_{i t}^{0}\right\}_{t=0}^{T}} E_{0}\left[\int_{0}^{T} e^{-\phi t} \frac{C_{i t}^{1-\frac{1}{\rho_{i}}}}{1-\frac{1}{\rho_{i}}} d t\right]
$$

subject to the budget constraint

$$
d W_{i t}=N_{i t}\left(d P_{t}+D_{t} d t\right)+N_{i t}^{0} B_{t} r_{t} d t-C_{i t} d t
$$

with initial condition $W_{i, 0}=s_{i} P_{0}$.

\subsection{Equilibrium}

To understand the logic of the equilibrium, it is useful to go through some of the steps (see Chan and Kogan (2002), Xiorious and Zapatero (2007)). Markets are dynamically complete and thus from Cox and Huang (1989) each agent $i$ equivalently solves the static maximization

$$
\max _{\left\{C_{i t}\right\}_{t=0}^{T}} E_{0}\left[\int_{0}^{T} e^{-\phi t} \frac{C_{i t}^{1-\frac{1}{\rho_{i}}}}{1-\frac{1}{\rho_{i}}} d t\right] \quad \text { subject to } \quad E_{0}\left[\int_{0}^{T} M_{t} C_{i t} d t\right]=s_{i} P_{0}
$$

where $M_{t}$ is the equilibrium state price density (normalized to $M_{0}=1$ ).

The Lagrangean of the static optimization is

$$
L_{i}=E_{0}\left[\int_{0}^{T} e^{-\phi t} \frac{C_{i t}^{1-\frac{1}{\rho_{i}}}}{1-\frac{1}{\rho_{i}}} d t\right]-\xi_{i}\left(E_{0}\left[\int_{0}^{T} M_{t} C_{i t} d t\right]-s_{i} P_{0}\right)
$$

where $\xi_{i}$ is the Lagrange multiplier determined by the static budget constraint. The maximization is taken state by state and time by time obtaining the first-order condition

$$
e^{-\phi t} C_{i t}^{-\frac{1}{\rho_{i}}}=\xi_{i} M_{t} \quad \Longrightarrow \quad C_{i t}=e^{-\rho_{i} \log \left(\xi_{i}\right)+\rho_{i} g_{t}}
$$

where

$$
g_{t}=-\phi t-\log \left(M_{t}\right)
$$


Aggregate now across agents and impose the market clearing condition $D_{t}=\int C_{i t} d i$ to obtain the equilibrium condition:

$$
D_{t}=\int e^{-\rho_{i} \log \left(\xi_{i}\right)+\rho_{i} g_{t}} d i=E^{C S}\left[e^{-\rho_{i} \log \left(\xi_{i}\right)+\rho_{i} g_{t}}\right]
$$

where the last step exploits the law of large numbers and $E^{C S}$ [.] denotes the cross-sectional average across agents $i{ }^{5}$ The quantity $g_{t}$, and hence the equilibrium state price density $M_{t}$, is the solution to equation (7), which I denote by

$$
g_{t}=g\left(\delta_{t}\right)
$$

The state price density is then

$$
M_{t}=e^{-\phi t-g\left(\delta_{t}\right)}
$$

Given the equilibrium $g\left(\delta_{t}\right)$, the constants $\xi_{i}$ are determined by the budget constraints (3). After substitution of $M_{t}$ and using $P_{0}=E_{0}\left[\int_{0}^{T} M_{\tau} D_{\tau} d \tau\right]=E_{0}\left[\int_{0}^{T} e^{-\phi \tau+\delta_{\tau}-g\left(\delta_{\tau}\right)} d \tau\right]$, the budget constraint (3) can be written as follows:

$$
e^{-\rho_{i} \log \left(\xi_{i}\right)}=s_{i} \lambda\left(\rho_{i}\right) \quad \text { where } \quad \lambda\left(\rho_{i}\right)=\frac{E_{0}\left[\int_{0}^{T} e^{-\phi \tau+\delta_{\tau}-g\left(\delta_{\tau}\right)} d \tau\right]}{E_{0}\left[\int_{0}^{T} e^{-\phi \tau+\left(\rho_{i}-1\right) g\left(\delta_{\tau}\right)} d \tau\right]}
$$

I finally substitute this expression back in equilibrium condition (7) to obtain:

$$
e^{\delta_{t}}=E^{C S}\left[s_{i} \lambda\left(\rho_{i}\right) e^{\rho_{i} g\left(\delta_{t}\right)}\right]
$$

The normalization $M_{0}=1$ implies $g\left(\delta_{0}\right)=0$. Assuming $\delta_{0}=0$ without loss of generality, I thus have the normalization

$$
E^{C S}\left[s_{i} \lambda\left(\rho_{i}\right)\right]=1
$$

Equations (9) and (10) highlight the nature of the functional fixed-point problem that characterizes an equilibrium: We need the equilibrium $g\left(\delta_{t}\right)$ to compute the Lagrange multipliers $\xi_{i}$ and hence $\lambda(\rho)$ from $(9)$ but we need $\lambda(\rho)$ to compute the equilibrium $g\left(\delta_{t}\right)$ from (10). Still, assuming that conditions are such that the fixed point has a solution, we can characterize the solution even without explicitly solving for $\lambda(\rho)$ (see Chan and Kogan (2002) and Xiorius and Zapatero (2007) for related results under habit formation):

\footnotetext{
${ }^{5}$ I assume throughout conditions are such that the law of large number can be applied. See Feldman and Gilles (1985), Judd (1985).
} 
Proposition 1: Let the functional fixed-point problem in Equations (9) and (10) have a solution for every $\delta_{t}$. Then:

(a) The solution $g\left(\delta_{t}\right)$ is unique

(b) The function $g\left(\delta_{t}\right)$ is globally increasing in $\delta_{t}$, it is concave, and it diverges to infinity as $\delta_{t}$ increases. Specifically:

$$
g^{\prime}\left(\delta_{t}\right)=\frac{1}{E_{t}^{*}[\rho]}>0 ; \quad g^{\prime \prime}\left(\delta_{t}\right)=g^{\prime}\left(\delta_{t}\right)\left[1-\frac{E_{t}^{* *}[\rho]}{E_{t}^{*}[\rho]}\right]<0
$$

where $E_{t}^{*}[$.$] and E_{t}^{* *}[$.$] denote cross-sectional averages computed using the following two$ distributions, respectively:

$$
f^{*}\left(\rho \mid \delta_{t}\right) \propto \int f(\rho, s) s d s \lambda(\rho) e^{\rho g\left(\delta_{t}\right)} ; \quad f^{* *}\left(\rho \mid \delta_{t}\right) \propto f^{*}\left(\rho \mid \delta_{t}\right) \rho
$$

(c) The function $g\left(\delta_{t}\right)>0$ if and only if $\delta_{t}>0$.

(d) The function $g^{\prime}\left(\delta_{t}\right)$ is bounded above and below:

$$
\frac{1}{\rho_{L}}>g^{\prime}\left(\delta_{t}\right)>\frac{1}{\rho_{H}}, \quad \lim _{\delta_{t} \rightarrow-\infty} g^{\prime}\left(\delta_{t}\right)=\frac{1}{\rho_{L}}, \quad \lim _{\delta_{t} \rightarrow \infty} g^{\prime}\left(\delta_{t}\right)=\frac{1}{\rho_{H}}
$$

Before I comment on Proposition 1, the following corollary ties it to the standard homogeneous case: 6

Corollary 1: If $\rho_{i}=\rho_{j}=\rho$ for all $i, j$, then $g\left(\delta_{t}\right)=\frac{1}{\rho} \delta_{t}$ and the state price density is $M_{t}=e^{-\phi t-\frac{1}{\rho} \delta_{t}}=e^{-\phi t} D_{t}^{-\frac{1}{\rho}}$.

Under homogeneous preferences we have the classic result that the state price density equals the marginal utility of the representative agent with risk aversion $1 / \rho$. Even in the homogeneous case we have $g^{\prime}\left(\delta_{t}\right)=1 / \rho>0$; and $\lim _{\delta \rightarrow \infty} g(\delta)=\infty$ and $g\left(\delta_{t}\right)>0$ for $\delta_{t}>0$.

Proposition 1 confirms that such properties are true also under heterogeneity. Indeed, note that $g^{\prime}\left(\delta_{t}\right)$ is the inverse of the average risk tolerance. Such average, however, is not computed from the original distribution of $\rho, f(\rho)$, but from a distorted distribution $f^{*}\left(\rho \mid \delta_{t}\right)$ that corrects the original one by the state-price density factor $g(\delta)$. As $\delta_{t}$ increases, $f^{*}\left(\rho \mid \delta_{t}\right)$ gives increasingly more weight to high $\rho$ (because $g\left(\delta_{t}\right)>0$ ) implying that the effective risk aversion $g^{\prime}\left(\delta_{t}\right)=1 / E^{*}\left[\rho \mid \delta_{t}\right]$ decreases as the economy gets stronger.

\footnotetext{
${ }^{6}$ The result is easy to see: When all agents are identical with risk tolerance $\rho, \lambda(\rho)=1$ for all $\rho$ and given $E^{C S}\left[s_{i}\right]=1$, equation (10) reduces to $\delta_{t}=\log \left(\left[e^{-\rho g\left(\delta_{t}\right)}\right]\right)=-\rho g\left(\delta_{t}\right)$.
} 
This effect is due to a combination of risk sharing and different elasticities of intertemporal substitution (EIS): Agents with lower risk aversion insure agents with higher risk aversion, and during good times they end up representing more of the aggregate consumption of the economy. In addition, such agents with higher risk tolerance have also higher EIS. Thus, they are happy to consume less in bad times but they want to consume more in good times. Agents with low risk tolerance, in contrast, want to smooth consumption over time and thus they want to consume more in bad times and relatively less in good times. With CRRA utility the two channels are interchangeable.

Although I did not use a representative agent set up to obtain the equilibrium, a representative agent exists in this economy, as shown in the next proposition:

Proposition 2. The function $g^{\prime}\left(\delta_{t}\right)$ is the relative risk aversion of the representative agent in this economy:

$$
g^{\prime}\left(\delta_{t}\right)=R R A(D)=-\frac{D \mathcal{U}^{\prime \prime}(D, t)}{\mathcal{U}^{\prime}(D, t)}
$$

where $\mathcal{U}(D, t)$ is the utility function of the representative agent

$$
\mathcal{U}(D, t)=e^{-\phi t} \int \frac{s_{i} \lambda\left(\rho_{i}\right) e^{\rho_{i} g(\log (D))}}{1-\frac{1}{\rho_{i}}} d i
$$

The representative agent's risk aversion is thus decreasing in output

$$
\frac{d R R A(D)}{d D}<0
$$

From Proposition 1, $g\left(\delta_{t}\right)$ is concave in $\delta_{t}$, as $g^{\prime}\left(\delta_{t}\right)$ decreases with $\delta_{t}$. The risk aversion of the representative agent hence decreases as the economy expands. Even if all agents have constant relative risk aversion, heterogeneity brings about a decrease in risk aversion in good times. As the economy grows, more weight is given to agents with lower risk aversion and the state price density reflects such lower weighted risk aversion. I further discuss this effect in the next section.

\subsection{Optimal Consumption}

The endogenous behavior of $g(\delta)$ has implications for the equilibrium consumption share of individual agents, i.e. their consumption-to-output ratio, which is given by: 7

$$
\frac{C_{i t}}{D_{t}}=s_{i} \lambda\left(\rho_{i}\right) e^{\rho_{i} g\left(\delta_{t}\right)-\delta_{t}}
$$

\footnotetext{
${ }^{7}$ See also Chan and Kogan (2002), Xiorius and Zapatero (2008) for a similar expression under habit formation.
} 
I then obtain the following:

\section{Corollary 2:}

(a) The $C / D$ ratio is increasing in output for agents with $\rho_{i}>E_{t}^{*}\left[\rho_{i}\right]$.

(b) Thus, as $\delta_{t}$ increases, so does the fractions of agents whose $C / D$ is declining in $\delta_{i}$.

Corollary 2 shows that for given $\delta_{t}$ agents with risk tolerance $\rho_{i}>E_{t}^{*}[\rho]$ have equilibrium $\mathrm{C} / \mathrm{D}$ ratios that increase as $\delta_{t}$ increases, and agents with low risk tolerance have $\mathrm{C} / \mathrm{D}$ ratios that decrease. The first types of agents eat an increasing larger share of aggregate output compared to the other types. However, interestingly, as output increases so does the effective average risk tolerance $E_{t}^{*}[\rho]$, as agents with higher risk tolerant agents weigh more in the distribution, and thus the threshold increases. It follows that the fraction of agents with declining $\mathrm{C} / \mathrm{D}$ ratio increases as well. That is, there is an increasing share of agents whose consumption does not keep up with the increase in aggregate output. This is the nature of risk-sharing with a continuum of heterogeneous agents. The exact mechanism which delivers this result (in terms of portfolio allocation) is discussed in Section 2.5.

We observe from expression (14) that the weights of the distribution $f^{*}(\rho \mid \delta)$ in Proposition 1 depend on consumption shares. In particular, denote $C_{t}(\rho, s)$ the time- $t$ consumption of an agent born with risk tolerance $\rho$ and an endowment of $s$ shares. Then:

$$
f^{*}(\rho \mid \delta)=\int f(\rho, s) \frac{C_{t}(\rho, s)}{D_{t}} d s
$$

This expression shows that the adjusted distribution on $\rho$ depends on the endowmentweighted consumption share of agents with risk tolerance $\rho$. For instance, even if $\rho$ was uniformly distributed, if agents with high $\rho$ consume more, then it is as if the average risk-tolerance of the economy is higher.

Corollary 3: Agents with higher risk tolerance $\rho_{i}$ have more volatile consumption. In particular:

$$
\frac{d C_{i t}}{C_{i t}}=\mu_{C, i} d t+\sigma_{C, i} d Z_{t}
$$

where

$$
\begin{aligned}
\mu_{C, i} & =\frac{\rho_{i}}{E_{t}^{*}[\rho]} \mu+\frac{1}{2} \frac{\rho_{i}}{E_{t}^{*}[\rho]}\left[1+\frac{\rho^{i}-E_{t}^{* *}[\rho]}{E_{t}^{*}[\rho]}\right] \\
\sigma_{C, i} & =\frac{\rho_{i}}{E_{t}^{*}[\rho]} \sigma
\end{aligned}
$$


Risk tolerance $\rho_{i}$ is also the elasticity of intertemporal substitution with CRRA utility. Higher elasticity of intertemporal substitution then makes agents have more volatile consumption (they are more elastic). Higher risk tolerance also implies a higher growth rate of consumption (from the first term), due to the willingness of smooth consumption over time as well and not only across states. The second term in $\mu_{C, i}$ is positive for high $\rho^{i}$ but it turns negative for low $\rho^{i}$ as $E_{t}^{* *}[\rho]$ increases over time.

Corollary 4: As the economy grows, the simple average consumption volatility declines

$$
\int \sigma_{C, i} f(\rho) d \rho=\frac{E\left[\rho_{i}\right]}{E_{t}^{*}[\rho]} \sigma
$$

This simple result shows that statistics that do not control for the density $f^{*}\left(\rho \mid \delta_{t}\right)$ would highlight properties of consumption volatility that are not real, but just an artifact of missweighting consumption growth.

\subsection{Asset Prices}

The interest rate and risk prices only depend on the state price density (8) and thus by Ito's Lemma I obtain the following proposition:

Proposition 3: The stochastic discount factor follows the process

$$
\frac{d M_{t}}{M_{t}}=-r_{t} d t-\sigma_{M, t} d Z_{t}
$$

where

$$
\begin{aligned}
r_{t} & =\phi+\frac{1}{E_{t}^{*}[\rho]}\left\{\mu-\frac{1}{2}\left(\frac{1+E_{t}^{* *}[\rho]-E_{t}^{*}[\rho]}{E_{t}^{*}[\rho]}\right) \sigma^{2}\right\} \\
\sigma_{M, t} & =\frac{\sigma}{E_{t}^{*}[\rho]}
\end{aligned}
$$

In the special case of $\rho_{i}=\rho=1 / \gamma$, then $E_{t}^{*}[\rho]=E_{t}^{* *}[\rho]=\rho$ and I obtain the standard result $r_{t}=\phi+\gamma \mu-\frac{\gamma^{2}}{2} \sigma^{2}$ and $\sigma_{M, t}=\gamma \sigma$.

From Proposition $1, E_{t}^{*}[\rho]$ increases with $\delta_{t}$, as more of the weights goes to higher agents with higher risk tolerance $\rho_{i}$, then risk prices $\sigma_{M, t}$ declines as the economy improves (see also Chan and Kogan (2002), Xiorius and Zapatero (2007)). 
The impact of growth on the real interest rate is non-linear. The last term in the parenthesis is declining in $\delta_{t}$ and converging to $\frac{\sigma^{2}}{2 \rho_{H}}$. Thus, if $\mu$ is sufficiently large interest rates decline as the economy becomes larger in size. Intuitively, agents with higher risk tolerance are also those with higher elasticity of intertemporal substitution (EIS). When the economy has positive growth such agents have lower desire to borrow to smooth out consumption compared to those with lower EIS. It follows that rates tend to decline as the economy grows. A countervailing effect, however, is that as the economy grows so does the average risk tolerance, and thus precautionary savings demand for bonds declines. This effect tends to raise interest rates. When $\mu$ is sufficiently large the first effect eventually dominates.

I next discuss the properties of the stock return process. Before I do so, I introduce the following notation for a generic function $K\left(\delta_{t+\tau}\right)$ of

$$
\delta_{t+\tau}=\delta_{t}+\mu \tau+\sigma \sqrt{\tau} x
$$

where $x \sim N(0,1)$ :

$$
\begin{aligned}
E_{t}^{x, \tau}\left[K\left(\delta_{t+\tau}\right)\right] & =E\left[\int_{0}^{T-t} \frac{\phi e^{-\phi \tau}}{1-e^{-\phi(T-t)}} K\left(\delta_{t+\tau}\right) d \tau \mid \delta_{t}\right] \\
& =\int_{0}^{T-t} \int_{-\infty}^{\infty} K\left(\delta_{t}+\mu \tau+\sigma \sqrt{\tau} x\right) n(x) f_{\tau}(\tau \mid T-t) d x d \tau
\end{aligned}
$$

where $n(x)$ is the standard normal density, $f_{\tau}(\tau \mid T-t)=\frac{\phi e^{-\phi \tau} 1_{\{\tau<T-t\}}}{1-e^{-\phi(T-t)}}$ is the density of a truncated random variable $\tau$ exponentially distributed over $[0, T-t]{ }^{8}$

Proposition 4: The stock price is

$$
P_{t}=p(T-t) e^{g\left(\delta_{t}\right)} E_{t}^{x, \tau}\left[e^{\delta_{t+\tau}-g\left(\delta_{t+\tau}\right)}\right]
$$

where $p(T-t)=\frac{1-e^{-\phi(T-t)}}{\phi}$. Thus, the stock return process

$$
\frac{d P_{t}+D_{t} d t}{P_{t}}=\mu_{P t} d t+\sigma_{P t} d Z_{t}
$$

where $\mu_{P t}=r_{t}+\sigma_{P t} \sigma_{M t}$, and

$$
\sigma_{P t}=\left\{1+g^{\prime}\left(\delta_{t}\right)-\widetilde{E}_{t}^{x, \tau}\left[g^{\prime}\left(\delta_{t+\tau}\right)\right]\right\} \sigma
$$

In this formula, $\widetilde{E}_{t}^{x, \tau}[$.$] denotes the expectation with respect to the following distribution$

$$
\tilde{f}\left(x, \tau \mid \delta_{t}\right) \propto n(x) f_{\tau}(\tau \mid T-t) e^{\delta_{t+\tau}-g\left(\delta_{t+\tau}\right)}
$$

\footnotetext{
${ }^{8}$ Indeed, $\int_{0}^{T-t} f_{\tau}(\tau \mid T-t) d \tau=\frac{\phi}{1-e^{-\phi(T-t)}} \int_{0}^{T-t} e^{-\phi \tau} d \tau=\frac{\phi}{1-e^{-\phi(T-t)}} \frac{e^{-\phi(T-t)}-1}{-\phi}=1$.
} 
Pricing formula (24) is a tautology from the definition of $E_{t}^{x, \tau}$ [.] and the pricing formula $P_{t}=E_{t}\left[\int_{t}^{T} M_{\tau} / M_{t} D_{\tau} d \tau\right]$. To understand the volatility formula, recall that

$$
g^{\prime}\left(\delta_{t}\right)=\frac{1}{E_{t}^{*}[\rho]}
$$

i.e. the inverse of the weighted average of risk tolerance. If all agents have $\rho_{i}=\rho=$ constant, then $E_{t}^{*}[\rho]=E_{t+\tau}^{*}[\rho]=\rho$ and thus I obtain the standard result $\sigma_{P t}=\sigma$. Indeed, in this case, the risk premium is simply $\sigma^{2} / \rho$. For instance, if $\rho=0.1$ then risk aversion is 10 and the risk premium is $10 \sigma^{2}$.

The existence of heterogeneity introduces time varying risk-tolerance, which in turn generates time varying volatility and expected return. The size and impact of the effect depends on whether the following condition is satisfied:

$$
g^{\prime}\left(\delta_{t}\right)>\widetilde{E}_{t}^{x, \tau}\left[g^{\prime}\left(\delta_{t+\tau}\right)\right]
$$

Next corollary explains why:

Corollary 5: (a) The price-dividend ratio

$$
\frac{P_{t}}{D_{t}}=p(T-t) e^{g\left(\delta_{t}\right)-\delta_{t}} E_{t}^{x, \tau}\left[e^{\delta_{t+\tau}-g\left(\delta_{t+\tau}\right)}\right]
$$

is increasing in $\delta_{t}$ if and only if condition (27) is satisfied.

(b) Condition (27) is satisfied if $\rho_{H}>1$ and $\delta_{t}$ is sufficiently high, or if the standardized growth rate of the economy $\mu / \sigma$ is sufficiently high.

If $\mathrm{P} / \mathrm{D}$ is increasing in $\delta_{t}$, then it is easy to see why the model with heterogeneous risk tolerance increases both the volatility and the risk premium compared to a model with homogeneous preferences. As $\delta_{t}$ increases, the stock price increases and the $\mathrm{P} / \mathrm{D}$ ratio also gets a positive kick due to a lower discount rate. The two effects compound each other and the stock price is more volatile and requires a higher risk premium compared to the case with homogeneous preferences.

However, the $\mathrm{P} / \mathrm{D}$ ratio may not be increasing in output $\delta_{t}$ if an increase in $\delta_{t}$ increases the risk-free rate, for instance. If the increase in weighted risk tolerance reduces sufficiently the precautionary savings demand for bonds, the interest rate may increase with $\delta_{t}$ which in turn may decrease the $\mathrm{P} / \mathrm{D}$ ratio. In this case, the volatility of stock returns would actually be lower than in the case with homogeneous investors. 


\subsection{Wealth and Wealth Dynamics}

Dynamic market completeness implies that the wealth of each agent is equal to the present value of his/her future consumption and thus

$$
W_{i t}=E_{t}\left[\int_{t}^{T} \frac{M_{s}}{M_{t}} C_{i s} d s\right]
$$

I now characterize agents' wealth as a function of aggregate (log) output $\delta_{t}$.

Proposition 5: The wealth of each agent $i$ at time $t$ is

$$
W_{i t}=p(T-t) s_{i} \lambda\left(\rho_{i}\right) E^{x, \tau}\left[e^{\left(\rho_{i}-1\right) g\left(\delta_{t+\tau}\right)}\right]
$$

and the consumption to wealth ratio $(C / W)$ is

$$
\frac{C_{i t}}{W_{i t}}=\frac{1}{p(T-t) E_{t}^{x, \tau}\left[e^{\left(\rho_{i}-1\right)\left(g\left(\delta_{t+\tau}\right)-g\left(\delta_{t}\right)\right)}\right]}
$$

Given (29) and (30), I can analyze a number properties of wealth and C/W ratio, even without knowing the exact functional form of $g(\delta)$ as solution of equation (11), but only its characterization in Proposition 1.

First, the random amount of initial shares endowment $s_{i}$ affects the level of wealth proportionally going forward. An agent with $10 \%$ more shares at time 0 due to a lucky draw of $s_{i}$ will be richer by $10 \%$ going forward. Indeed, the consumption/wealth ratio does not depend on $s_{i}$.

Second, the dynamics of output $\delta_{t}$ affects the level of wealth of each agent differently depending on their risk tolerance. For instance, the log-utility agent $\left(\rho_{i}=1\right)$ has wealth

$$
W_{i t}^{\rho_{i}=1}=s_{i} \lambda(1) e^{g\left(\delta_{t}\right)} \frac{1-e^{-\phi(T-t)}}{\phi}
$$

Thus, an increase in output increases the log-utility agent's wealth. However, if $E_{t}^{*}(\rho)>1$, the wealth to output ratio $(\mathrm{W} / \mathrm{D})$ of log-utility agent declines with output when $\delta_{t}$ is large enough. Indeed,

$$
\frac{d \log \left(W_{i t}^{\rho_{i}=1} / D_{t}\right)}{d \delta_{t}}=g^{\prime}(\delta)-1<0 \Leftrightarrow E_{t}^{*}[\rho]>1 .
$$

Assuming $\rho_{H}>1$, because $E_{t}^{*}[\rho] \rightarrow \rho_{H}$, there is a point in which $E_{t}^{*}[\rho]>1$ and W/D of log-utility investor declines with output. Clearly, the consumption/wealth ratio of log utility agent - a myopic agent - is still deterministic and independent of output, as we would expect

$$
\frac{C_{i t}^{\rho_{i}=1}}{W_{i t}^{\rho_{i}=1}}=\frac{\phi}{1-e^{-\phi(T-t)}}
$$


I obtain the following:

Corollary 6: When $\delta_{t}$ is sufficiently high, for given $s_{i}$, agents with higher risk tolerance $\rho_{i}$ have more wealth than agents with lower risk tolerance.

It is easy to see how Corollary 6 stems from expression (29) and Proposition 3. Indeed, recall that $s_{i} \lambda\left(\rho_{i}\right)=e^{-\rho_{i} \log \left(\xi_{i}\right)}$. After substitution, the claim then follows from the fact that $g\left(\delta_{t+\tau}\right)-\log \left(\xi_{i}\right)>0$ for any $\xi_{i}$ when $\delta_{t+\tau}$ is sufficiently high. Because $\delta_{t+\tau}$ is linear in $\delta_{t}$, as the latter increases so does the distribution of $\delta_{t+\tau}$, yielding the result.

The same argument implies the following:

Corollary 7: Agents with higher risk tolerance consume a lower percentage of their wealth than agents with lower risk tolerance.

This is easy to see as well: we know that $\left(g\left(\delta_{t+\tau}\right)-g\left(\delta_{t}\right)\right)>0$ for $\delta_{t+\tau}>\delta_{t}$. Therefore, in a growing economy we have a large part of the distribution with $g\left(\delta_{t+\tau}\right)-g\left(\delta_{t}\right)>0$. Thus,

a higher $\rho_{i}$ implies higher $E_{t}^{x, \tau}\left[e^{\left(\rho_{i}-1\right)\left(g\left(\delta_{t+\tau}\right)-g\left(\delta_{t}\right)\right)}\right]$ and therefore lower $\mathrm{C} / \mathrm{W}$. Moreover, as $\delta_{t}$ increases concavity implies that the difference $\left(g\left(\delta_{t+\tau}\right)-g\left(\delta_{t}\right)\right)$ decreases, and therefore there is a compression of $\mathrm{C} / \mathrm{W}$ ratios as the economy grows stronger (for given $T-t$ ).

To discuss further properties, it is useful to first normalize the wealth of each agent by the aggregate wealth (which equal the stock market price, by market clearing).

$$
P_{t}=\int W_{j t} d j=p(T-t) E^{C S}\left[s_{j} \lambda\left(\rho_{j}\right) E^{x, \tau}\left[e^{\left(\rho_{j}-1\right) g\left(\delta_{t+\tau}\right)}\right]\right]
$$

\section{Corollary 8:}

(a) The wealth distribution is given by weights

$$
\omega_{i t}=\frac{W_{i, t}}{P_{t}}=\frac{s_{i} \lambda\left(\rho_{i}\right) E_{t}^{x, \tau}\left[e^{\left(\rho_{i}-1\right) g\left(\delta_{t+\tau}\right)}\right]}{E^{C S}\left[s_{j} \lambda\left(\rho_{j}\right) E_{t}^{x, \tau}\left[e^{\left(\rho_{j}-1\right) g\left(\delta_{t+\tau}\right)}\right]\right]}
$$

(b) The initial weights at time 0 are $\omega_{i 0}=s_{i}$.

(c) Agent $i$ 's wealth share $\omega_{i t}$ increases with output if and only if

$$
\left(\rho_{i}-1\right) \frac{E_{t}^{x, \tau}\left[g^{\prime}\left(\delta_{t+\tau}\right) e^{\left(\rho_{i}-1\right) g\left(\delta_{t+\tau}\right)}\right]}{E_{t}^{x, \tau}\left[e^{\left(\rho_{i}-1\right) g\left(\delta_{t+\tau}\right)}\right]}>1-\widetilde{E}_{t}^{x, \tau}\left[g^{\prime}\left(\delta_{t+\tau}\right)\right]
$$

where recall $\widetilde{E}_{t}^{x, \tau}[$.$] uses density (26)$ 
(d) In addition, for any cutoff $\underline{\rho}$, the wealth share of agents $i$ with $\rho_{i}>\underline{\rho}$ increases as $\delta_{t}$ increases. Denote $\omega_{t}(\underline{\rho})=\int_{i: \rho_{i}>\underline{\rho}} \omega_{i t} d i$. Then

$$
\frac{\partial \omega_{t}(\underline{\rho})}{\partial \delta_{t}}>0
$$

Equation (32) shows the two sources of wealth distribution, namely, the initial random endowment $s_{i}$ and the component due to risk preferences (and their implicit implication for sharing). The second ratio in (32) can in fact be characterized and it depends on $\rho_{i}$ and the level of current output $\delta_{t}$. When $\delta_{t}$ increases, the wealth share of the top distribution of risk tolerance $\rho>\underline{\rho}$ increases, for any $\underline{\rho}$ (point (d)). This implies of course that the wealth share of the ones below the cutoff worsen. Point (c) shows the condition under which the wealth share of agent $i$ increases. The right-hand-side of (33) does not depend on $i$ and it is increasing in $\delta_{t}$. Unfortunately, the left-hand-side is also increasing in $\delta_{t}$, unless $\rho=1$, in which case this becomes a constant. It follows that a log utility investor has its wealth share increase for a while and then decline as the economy grows (assuming $\rho_{H}>1$ ).

I finally characterize the dynamics of wealth:

Proposition 6: Agent i's wealth dynamics is

$$
\frac{d W_{i t}+C_{i t} d t}{W_{i t}}=\mu_{W i t} d t+\sigma_{W i t} d Z_{t}
$$

where $\mu_{W i t}=r_{t}+\sigma_{W i t} \sigma_{M t}$ and

$$
\sigma_{W i t}=\left\{g^{\prime}\left(\delta_{t}\right)+\left(\rho_{i}-1\right) \frac{E^{x, \tau}\left[g^{\prime}\left(\delta_{t+\tau}\right) e^{\left(\rho_{i}-1\right) g\left(\delta_{t+\tau}\right)}\right]}{E^{x, \tau}\left[e^{\left(\rho_{i}-1\right) g\left(\delta_{t+\tau}\right)}\right]}\right\} \sigma
$$

Before discussing Proposition 6, I provide a second characterization to the stock price, based on (31):

Corollary 9: The stock return volatility and expected return can be expressed as

$$
\begin{aligned}
\sigma_{P t} & =\int \omega_{i t} \sigma_{W i t} d i \\
\mu_{P t} & =r_{t}+\int \omega_{i t} \mu_{W i t} d i
\end{aligned}
$$

The volatility and risk premium of stock returns are wealth-weighted averages of the volatility and risk premium of the wealth process of individual agents in the economy. I thus have the following proposition: 


\section{Proposition 7:}

(a) There is a threshold $\rho^{*}>1$ such that for all $\rho_{i}<\rho^{*}$ agents with higher $\rho_{i}$ have higher volatility of wealth $\sigma_{W i}$ :

$$
\rho_{i}<\rho_{j} \Rightarrow \sigma_{W i t}<\sigma_{W j t}
$$

(b) Let $\rho_{H} \leq \rho^{*}$ and let the marginal distribution $f(\rho)$ be continuous on $\left(\rho_{L}, \rho_{H}\right)$. For every $\delta_{t}$ there is $\rho\left(\delta_{t}\right)$ such that

$$
\sigma_{W i t}<\sigma_{P t}<\sigma_{W j t} \quad \text { for every } i \text { and } j \text { such that } \rho_{i}<\rho\left(\delta_{t}\right)<\rho_{j}
$$

The condition in Proposition 7 that there is $\rho^{*}>1$ is not necessary but just sufficient. It could well be that $\rho^{*}=\infty$ but the general case proved tricky to establish formally given the generality of the distribution $f(\rho)$. From an economic standpoint, agents with higher risk tolerance have higher volatility of wealth. Intuitively, such agents are more willing to have higher consumption in good times and lower consumption in bad times (because they insure the agents with lower risk tolerance). That is, the consumption of such agents is more "volatile" than consumption of more risk averse agents, as shown in Corollary 3. It follows that their wealth - the present value of such consumption streams - respond more to aggregate shocks as their cash flow volatility is more volatile.

Part (b) stems from the fact that the average is always strictly between the extremes and $\sigma_{W i t}$ is increasing in $\rho_{i}$. This result is important to understand the "beta" of the wealth portfolio and the trading positions of agents in this economy, as discussed next.

Proposition 8: Let $\rho_{H}<\rho^{*}$ where $\rho^{*}$ is the threshold in Proposition 7 . Then, households with higher risk tolerance have a higher "beta" of their wealth dynamics. Let

$$
\beta_{i t}=\frac{\operatorname{Cov}_{t}\left(d W_{i t} / W_{i t}, d P_{t} / P_{t}\right)}{\operatorname{Var}_{t}\left(d P_{t} / P_{t}\right)}
$$

then

$$
\rho_{i}<\rho\left(\delta_{t}\right)<\rho_{j} \Rightarrow \beta_{i t}<1<\beta_{j t}
$$

where $\rho\left(\delta_{t}\right)$ is the threshold in Proposition \%, point (b).

That is, households with higher risk tolerance have a higher sensitivity of their wealth to the stock market. Because such households also tend to grow richer than others (see Corollary 6), we obtain the prediction that richer households tend to have wealth dynamics that is more sensitive to the stock market. Indeed, this point can also be seen from a "CAPM" perspective. The risk premium of household $i$ 's wealth is in fact

$$
\mu_{W i t}=\sigma_{W i t} \sigma_{M t}=\frac{\sigma_{W i t}}{\sigma_{P t}} \mu_{P t}=\beta_{i t} \mu_{P t}
$$


That is, the rate of return on wealth is higher for households with higher "beta" and hence, given Proposition 8, for agents with high risk tolerance. Because everything else equal, agents with high risk tolerance become richer in the long run (see Corollary 6), Proposition 8 lends support to the empirical evidence that richer households have net worth with higher beta (see e.g. Gomez (2018)). Next section ties these results with households' optimal portfolio allocations.

\subsection{Portfolio Allocation}

I use the results in Cox and Huang (1986) and the properties of dynamic market completeness to obtain the relative positions of agents in stocks and bonds. The wealth of agent $i$ can be written as a portfolio of stocks and bonds:

$$
W_{i t}=N_{i t} P_{t}+N_{i t}^{0} B_{t}
$$

where $B_{t}$ is the value of the bond. We then must have

$$
d W_{i t}=N_{i t} d P_{t}+N_{i t}^{0} d B_{t}
$$

From Ito's lemma, we thus have

$$
o(d t)+\sigma_{W i t} W_{i t} d Z_{t}=o(d t)+N_{i t} \sigma_{P t} P_{t} d Z_{t}
$$

Equating the diffusion terms, the position in stocks must be given by

$$
N_{i t}=\frac{\sigma_{W i t}}{\sigma_{P t}} \frac{W_{i t}}{P_{t}}
$$

Given $N_{i t}$, I can determine the position in bonds as a residual

$$
N_{i t}^{0} B_{t}=W_{i t}-N_{i t} P_{t}=W_{i t}\left(1-\frac{\sigma_{W i t}}{\sigma_{P t}}\right)
$$

The model has thus implications on the trading strategy of agents which depend on the level of the economy $\delta_{t}$.

Proposition 9: Let $\rho_{H}<\rho^{*}$ and let $\rho\left(\delta_{t}\right)$ be threshold defined in Proposition 7 . Then investors with higher risk tolerance $\rho_{i}>\rho\left(\delta_{t}\right)$ take on leverage:

$$
N_{i t}^{0} B_{i t}<0 \quad \text { if and only if } \quad \rho_{i}>\rho\left(\delta_{t}\right)
$$

Moreover, investors who lever invest more than $100 \%$ of their wealth in risky assets

$$
\frac{N_{i t} P_{t}}{W_{i t}}>1 \quad \text { if and only if } \quad \rho_{i}>\rho\left(\delta_{t}\right)
$$


Proposition 9 provides the economic intuition of the earlier results: Agents with high risk tolerance borrow from the other agents to invest in the risky security. The risky security pays off in good times (when $\delta_{t}$ increases) which in turn make such agents wealthier and make them consume more. It is all endogenous.

Proposition 7 and 9 also shows that the identity of agents who take on leverage changes over time. While general results seems difficult due to the complexity of formulas, I can provide some partial results around log utility $\rho=1$.

Proposition 10: Let the marginal distribution $f(\rho)$ be defined over $\left(\rho_{L}, \rho_{H}\right)$ with $\rho_{L}<1<$ $\rho_{H}$. A log-utility investor leverage if and only if ${ }^{9}$

$$
\widetilde{E}_{t}^{x, \tau}\left[g^{\prime}\left(\delta_{t+\tau}\right)\right]>1
$$

Condition (38) is satisfied for $\delta_{t}$ sufficiently low but it is violated for $\delta_{t}$ sufficiently high. Thus, the log-utility investor moves from being a borrower to a lender as the economy grows.

The proposition shows that for sufficiently low $\delta_{t}$, the log-utility investor is among those with higher risk tolerance, and thus that investor will leverage its position. However, as the economy grows and the wealth shifts towards the agents with even higher risk tolerance $(\rho>1)$, such an investor ends up being among those that are not leveraged. Intuitively, the Sharpe ratio keeps declining and even log-utility investors find it optimal to hold some bonds, eventually.

While proving a more general proposition seems hard, by continuity, the proof holds for a whole set of agents around $\rho=1$. In other words, the agents who take leverage are different as $\delta_{t}$ increases. The conjecture is that the fraction of agents who lever shrinks over time as stock return volatility increases. I find such result in my calibration in Section 4.

\subsection{The Dispersion of Risk Tolerance}

In this section, I study the impact of the properties of the distribution $f(\rho)$ on asset prices. For simplicity, let $s_{i}=1$ for all $i$, that is, all agents have the same initial endowment. Let $f(\rho)$ be the distribution of $\rho$. We then have the following:

Corollary 10: Consider two distributions $f_{1}(\rho)$ and $f_{2}(\rho)$ with $f_{2}$ stochastically dominating

\footnotetext{
${ }^{9}$ Recall that $\widetilde{E}_{t}^{x, \tau}[$.$] uses distribution (26).$
} 
$f_{1}$. Then $E_{2}^{*}[\rho \mid \delta]>E_{1}^{*}[\rho \mid \delta]$. Therefore, risk prices $\sigma_{M, t}$ is lower under $f_{2}$ than under $f_{1}$. Finally, if $\mu / \sigma$ is sufficiently high, then the risk free rate $r_{t}$ is lower under $f_{2}$ than under $f_{1}$.

That is, if $f_{2}(\rho)$ gives uniformly more mass to higher risk tolerance agents than $f_{1}(\rho)$, then the weighted average risk tolerance increases. The result is intuitive.

I now study the impact of the dispersion of risk-tolerance on asset prices. How does an increase in the dispersion of the distribution $f(\rho)$ affect consumption, wealth, and asset prices? I analyze the dispersion through a mean-preserving spread to $f(\rho)$.

Proposition 11. Let $f_{m p s}(\rho)$ be a mean preserving spread on $f(\rho)$. If $\mu / \sigma$ is sufficiently high, then there are thresholds $\delta_{\min }$ and $\delta_{\max }$ with $0<\delta_{\min }<\delta_{\max }$ such that the weighted risk tolerance $E_{m p s}^{*}\left[\rho \mid \delta_{t}\right]<E^{*}\left[\rho \mid \delta_{t}\right]$ for $\delta_{t}<\delta_{\min }$ and $E_{m p s}^{*}\left[\rho \mid \delta_{t}\right]>E^{*}\left[\rho \mid \delta_{t}\right]$ for $\delta_{t}>\delta_{\max }$.

Proposition 11 establishes that the risk adjusted risk tolerance $E^{*}[\rho \mid \delta]$ decreases for low $\delta_{t}$ when we increase the dispersion of risk tolerance, but it increases for high $\delta_{t}$. This result leads to the following implication:

Corollary 11. Under the conditions of Proposition 11, a mean preserving spread on the distribution of risk-tolerance increases the market price of risk and the risk-free rate for low output $\delta_{t}<\delta_{\min }$ but decrease them for high output $\delta_{t}>\delta_{\max }$.

Essentially, an increase in the dispersion of risk tolerance makes some agents more risk averse and some agents less risk averse than the average (which is kept constant). For low $\delta_{t}$, the impact of more risk averse ones is larger than the impact of less risk averse agents and thus the consumption/adjusted average risk tolerance decreases. This effect increases risk prices. In addition, because the same effect decreases the elasticity of intertemporal substitution, more agents want to borrow, and hence the interest rate increases. However, as the economy grows, at some point the increase in the mass of agents with lower risk tolerance becomes a dominant effect, and thus risk prices and interest rate decline by more than under the original distribution.

The impact on asset prices of a mean-preserving spread on the distribution of risk tolerance is harder to determine. However, the $\mathrm{P} / \mathrm{D}$ ratio formula (28) indicates that the $\mathrm{P} / \mathrm{D}$ ratio depends on the expected difference $E_{t}^{x, \tau}\left[e^{g\left(\delta_{t}\right)-g\left(\delta_{t+\tau}\right)}\right]$. When $g^{\prime}(\delta)$ is higher, this expected difference is higher, and thus we can expect the $\mathrm{P} / \mathrm{D}$ ratio to be lower. While harder to prove formally, this argument strongly suggests that a mean preserving spread on risk tolerance decreases the $\mathrm{P} / \mathrm{D}$ ratio. 


\subsection{Initial Endowments and Asset Prices}

I finally discuss the impact of different initial endowments on assets prices. That is, what happens to asset prices when we shift wealth from e.g. low risk averse to high risk averse agents? This shift is likely more prevalent than we think in the real world: Redistributive policies that shift wealth from rich to poor likely shift it from agents with low risk aversion to those with high risk aversion, as the former are those that become richer in good times.

Indeed, the previous sections show that agents with high risk tolerance $\rho$ eventually become richer than agents with low risk tolerance. I now study the impact of initial endowments that depend on risk tolerance $\rho$ :

Proposition 12: Let $s_{i}=\bar{s}(\rho) \tilde{s}_{i}$ where $\bar{s}(\rho)$ is monotonic in $\rho$ such that $E^{C S}[\bar{s}(\rho)]=1$ and $\tilde{s}_{i}$ is a unit-mean positive random noise term uncorrelated with $\rho_{i}$. Then if $\mu / \sigma$ is sufficiently high, for any two functions $\bar{s}_{1}(\rho)$ and $\bar{s}_{2}(\rho)$,

$$
\bar{s}_{1}^{\prime}(\rho)>\bar{s}_{2}^{\prime}(\rho) \Longrightarrow E_{1}^{*}[\rho \mid \delta]>E_{2}^{*}[\rho \mid \delta]
$$

Proposition 12 verifies that if we give a higher endowment to low risk tolerant agents at time zero, then the adjusted average risk-tolerance in the economy decreases. That is, redistributive policies that transfer funds from high risk tolerant agents to low risk tolerant agents have the implicit impact of decreasing the weighted average risk tolerance, and hence risk prices, as implied by the following corollary:

Corollary 12: Under the conditions of Proposition 12:

$$
\bar{s}_{1}^{\prime}(\rho)>\bar{s}_{2}^{\prime}(\rho) \Longrightarrow \sigma_{M t, 1}<\sigma_{M t, 2}
$$

Most developed economies have tax and subsidy policies that implicitly transfer wealth from rich agents to poor agents. Given that wealth is endogenous, however, in my model the rich agents are those that likely are less risk averse than the poor agents. Consequently, the transfer of wealth from rich to poor amount to an increase in wealth of more risk averse agents and thus an increase in the risk premium. 


\section{Macroeconomic Uncertainty and Learning}

I now introduce macroeconomic uncertainty and learning. The additional assumption is simple: Let's assume that the drift rate of output growth $\mu$ is not known but distributed according to a generic prior distribution $\pi_{0}(\mu)$ :

$$
\mu \sim \pi_{0}(\mu)
$$

Because I am interested in studying the possibility of an increase in uncertainty, I do not take $\pi_{0}(\mu)$ to be normally distributed because the properties of the Kalman-Bucy filter would imply that uncertainty decreases deterministically. Other non-normal prior distributions $\pi_{0}(\mu)$ may generate an increase of uncertainty for some periods of time.

Even if $\pi_{0}(\mu)$ is a general prior distribution over the parameter $\mu$, I can still characterize the dynamics of the posterior density of $\pi_{t}(\mu)$.

Lemma: Let $\pi_{0}(\mu)$ be such that $\int \pi_{0}(\mu) d \mu=1$ and $\pi_{0}(\mu)>0$. Then for every $\mu$, the density $\pi_{t}(\mu)$ follows the martingale process:

$$
d \pi_{t}(\mu)=\pi_{t}(\mu)\left(\mu-E_{t}[\mu]\right) \sigma^{-1} d \widehat{Z}_{t}
$$

where

$$
\begin{aligned}
E_{t}[\mu] & =\int \mu \pi_{t}(\mu) d \mu \\
d \widehat{Z}_{t} & =\sigma^{-1}\left(d \delta_{t}-E_{t}[\mu] d t\right)
\end{aligned}
$$

In addition, $\pi_{t}(\mu)>0$ and $\int \pi_{t}(\mu) d \mu=1$ almost surely.

That is, we can easily track the whole distribution $\pi_{t}(\mu)$ over time. From (41) we can rewrite the dividend process under the information filtration as

$$
d \delta_{t}=E_{t}[\mu] d t+\sigma d \widehat{Z}_{t}
$$

It follows that all state variables depend only on the innovation process $d \widehat{Z}_{t}$ and thus markets are dynamically complete. Therefore, the results in the previous section extend immediately to this case, as we show next. The benefit of this setting is that we can then investigate the implications of learning and stochastic changes in uncertainty and asset prices and trading.

\subsection{Asset Prices under Parameter Uncertainty}

The derivations in Section 2.2. highlight that the results in Propositions 1 and 2, and Corollaries 1 and 2 also hold under uncertainty and learning about $\mu$. Indeed, $\mu$ does not enter anywhere in those derivations or expressions because of time-separable preferences and 
market completeness. The consumption dynamics in Corollary 3, Equation (16), is almost identical, except that $E_{t}[\mu]$ replaces $\mu$ (which is not observable) in the formula for expected consumption growth (17), and the shock to households' consumption growth is the Brownian motion $d \widehat{Z}_{t}$ in (41) instead of the unobservable $d Z_{t}$.

The state price density $M_{t}$ in Proposition 3 also only depends on $\delta_{t}$, which is observable, and not on the parameter $\mu$. Thus, parameter uncertainty does not affect $M_{t}$ either. The stochastic discount factor $d M_{t} / M_{t}$ in Proposition 3, Equation (19), is only mildly affected as the interest rate $r_{t}$ in (20) now depends on $E_{t}[\mu]$ instead of $\mu$ and the shock to the SDF is now $d \widehat{Z}_{t}$ instead of $d Z_{t}$. The risk price $\sigma_{M t}$ in $(21)$ is unaffected.

In contrast to households' consumption and the state-price density, the stock price is affected by the unobservable $\mu$, as the latter affects the expectation $E_{t}^{x, \tau}[$.$] . However, it only$ amounts to a change of the definition of the expectation itself. For any function $K\left(\delta_{t+\tau}\right)$ where $\delta_{t+\tau}=\delta_{t}+\mu \tau+\sigma \sqrt{\tau} x$ we can define $E_{t}^{\mu, x, \tau}\left[K\left(\delta_{t+\tau}\right)\right]$ as in (23) except that the expectation is taken also over $\mu \sim \pi_{t}(\mu)$ in addition to the normal density $n(x)$ and the density $f_{\tau}(\tau \mid \tau<T) .{ }^{10}$ We thus obtain the following modification to Proposition 4 :

Proposition 13: The stock price is

$$
P_{t}=p(T-t) e^{g\left(\delta_{t}\right)} E_{t}^{\mu, x, \tau}\left[e^{\delta_{t+\tau}-g\left(\delta_{t+\tau}\right)}\right]
$$

where $p(T-t)=\frac{1-e^{-\phi(T-t)}}{\phi}$. Thus, the stock return process

$$
\frac{d P_{t}+D_{t} d t}{P_{t}}=\mu_{P t} d t+\sigma_{P t} d \widehat{Z}_{t}
$$

where $\mu_{P t}=r_{t}+\sigma_{P t} \sigma_{M t}$ and

$$
\sigma_{P t}=\left\{1+g^{\prime}\left(\delta_{t}\right)-\widetilde{E}_{t}^{\mu, x, \tau}\left[g^{\prime}\left(\delta_{t+\tau}\right)\right]\right\} \sigma+\left(\widehat{E}_{t}[\mu]-E_{t}[\mu]\right) \sigma^{-1}
$$

In this formula, $\widetilde{E}_{t}^{\mu, x, \tau}[$.$] denotes the expectation with respect to the distribution$

$$
\tilde{f}\left(\mu, x, \tau \mid \delta_{t}\right) \propto \pi_{t}(\mu) n(x) f_{\tau}(\tau) e^{\delta_{t+\tau}-g\left(\delta_{t+\tau}\right)}
$$

and $\widehat{E}_{t}[\mu]$ uses the modified distribution

$$
\widehat{\pi}_{t}(\mu) \propto \pi_{t}(\mu) E_{t}^{x, \tau}\left[e^{\delta_{t+\tau}-g\left(\delta_{t+\tau}\right)} \mid \mu\right]
$$

The price formula (42) is the direct extension of the one under certainty in expression (24). The next corollary follows:

\footnotetext{
${ }^{10}$ As shown in Section 4., such expectation is straightforward to compute by Monte Carlo simulations.
} 
Corollary 13: A mean-preserving spread on the distribution $\pi_{t}(\mu)$ increases the stock price $P_{t}$. That is, higher macroeconomic uncertainty increases stock valuations.

It is easy to see that the parameter $\mu$ enters linearly into $\delta_{t+\tau}$ and $e^{\delta_{t+\tau}-g\left(\delta_{t+\tau}\right)}$ is convex in $\delta_{t+\tau}$ and hence in $\mu$. A mean preserving spread over a convex function increases its expected value. This result is related to the analogous result under homogeneous preferences (see Veronesi (2000, proof of Lemma 3) and also Appendix to Pastor and Veronesi (2006)). In particular, Pastor and Veronesi (2006) use a similar convexity result to explain the high valuations in the late 1990s, a point I will return to in Section 4.

The volatility formula (43) under learning is similar to the one under certainty in Proposition 4 , except that we have the additional learning-related term $\left(\widehat{E}_{t}[\mu]-E_{t}[\mu]\right) \sigma^{-1}$. Fortunately, as in Veronesi (2000), I can provide a sign to this term depending on whether $E_{t}^{x, \tau}\left[e^{\delta_{t+\tau}-g\left(\delta_{t+\tau}\right)} \mid \mu\right]$ is increasing or decreasing in $\mu$. This in turn depends on the distribution of risk tolerance $\rho$ and the current output $\delta_{t}$.

Corollary 14: (a) If $\rho_{H}<1$, then $\widehat{E}_{t}[\mu]<E_{t}[\mu]$; (b) If $\rho_{H}>1$ and $\delta_{t}$ is sufficiently large, then $\widehat{E}_{t}[\mu]>E_{t}[\mu]$

Characterizing the sign of $\widehat{E}_{t}[\mu]-E_{t}[\mu]$ is not sufficient to also sign the full impact of learning on volatility and risk premium, however, because uncertainty also impacts the term $\widetilde{E}_{t}^{\mu, x, \tau}\left[g^{\prime}\left(\delta_{t+\tau}\right)\right]$. We will see in the numerical example that the second term dominates the impact of learning on asset returns.

Moving to economics, as in Veronesi (2000), if all agents have EIS $<1$ (part (a)), then higher growth $\mu$ always implies a lower $\mathrm{P} / \mathrm{D}$ ratio. Thus, the diffusion of stock returns is smaller under learning because a positive shock to dividends increases the expected future growth which in turn reduces the $\mathrm{P} / \mathrm{D}$ ratio. Consequently, while the positive shock increases the price directly because of higher dividend rate, the increase in expected growth reduces the $\mathrm{P} / \mathrm{D}$ ratio (for given discount) and thus reduces the impact of the positive shock.

If $\rho_{H}>1$, however, and the economy is growing, at some point the weight on high EIS agents is sufficient to imply that an increase in expected growth rate increases the $\mathrm{P} / \mathrm{D}$ ratio. This additional kick to prices will increase the volatility of stocks. Unfortunately, the risk premium at this point would still be low because risk prices are low. ${ }^{11}$

\footnotetext{
${ }^{11} \mathrm{~A}$ popular expedient to obtain higher volatility and thus higher risk-premium is to assume that equity is leveraged. Following Abel (1990), the price of a security that pays $D_{L, t}=D_{t}^{\alpha}$ for $\alpha>0$ not only has higher volatility on its own, but it generate a $\mathrm{P} / \mathrm{D}$ ratio that is increasing in $\mu$ if $\alpha$ is sufficiently high. As such, the volatility and equity premium of such security would be enhanced substantially.
} 


\subsection{Wealth Dynamics under Uncertainty}

Under parameter uncertainty, the wealth level and its dynamics are immediately related to their counterparts in Propositions 4 and 5:

Proposition 14: The wealth of each agent $i$ at time $t$ is

$$
W_{i t}=p(T-t) s_{i} \lambda\left(\rho_{i}\right) e^{g\left(\delta_{t}\right)} E_{t}^{\mu, x, \tau}\left[e^{\left(\rho_{i}-1\right) g\left(\delta_{t+\tau}\right)}\right]
$$

and the wealth dynamics is

$$
\frac{d W_{i t}+C_{i t} d t}{W_{i t}}=\mu_{W i t} d t+\sigma_{W i t} d \widehat{Z}_{t}
$$

where $\mu_{W i t}=r_{t}+\sigma_{W i t} \sigma_{M t}$ and

$$
\sigma_{W i, t}=\left\{g^{\prime}\left(\delta_{t}\right)+\left(\rho_{i}-1\right) \frac{E_{t}^{\mu, x, \tau}\left[g^{\prime}\left(\delta_{t+\tau}\right) e^{\left(\rho_{i}-1\right) g\left(\delta_{t+\tau}\right)}\right]}{E_{t}^{\mu, x, \tau}\left[e^{\left(\rho_{i}-1\right) g\left(\delta_{t+\tau}\right)}\right]}\right\} \sigma+\left(\widehat{E}_{t}^{i}[\mu]-E_{t}[\mu]\right) \sigma^{-1}
$$

where $\widehat{E}^{i}[$.$] uses the distribution$

$$
\widehat{\pi}_{t}^{i}(\mu) \propto \pi_{t}(\mu) E^{x, \tau}\left[e^{\left(\rho_{i}-1\right) g\left(\delta_{t+\tau}\right)} \mid \mu\right]
$$

The volatility of wealth (48) is similar to the counterpart in equation (34) without learning, except for the last term, which depends on parameter uncertainty. Fortunately, we can easily see whether this term is positive or negative, and it depends on risk tolerance:

Corollary 15: $\widehat{E}_{t}^{i}[\mu]>E_{t}[\mu]$ if and only if $\rho_{i}>1$.

Corollary 15 suggests that parameter uncertainty increases wealth volatility of agents that are more risk-tolerant than log-utility, while it decreases wealth volatility of investors that are less risk-tolerant than log. It is harder to prove the general statement because parameter uncertainty also affects the second term in equation (48), which is harder to sign. Still, to first order, it seems that introducing learning increases the volatility of wealth for agents that are more risk tolerant than log. Indeed, we can instead prove the following:

Corollary 16: The wealth volatility of log-utility investors is not affected by parameter uncertainty.

If all investors in the economy are less risk tolerant than $\log$ (i.e. $\rho_{H}<1$ ), the all households have lower wealth volatility under learning than under certainty. Although such result appears paradoxical, it is of course related to the same result already highlighted for stocks and discussed in Veronesi (2000). 


\subsection{Portfolio Allocation under Uncertainty}

Because markets are still dynamically complete under learning, the allocations to stocks and bonds are still given by expressions (37) and (38). To better characterize how the change in uncertainty affects trading behavior, I consider again a mean-preserving spread on the distribution $\pi_{t}(\mu)$. General results are hard to obtain in this case, but I can prove the following:

Proposition 15: Let $\tilde{\pi}_{t}(\mu)$ be a mean-preserving-spread on the distribution $\pi_{t}(\mu)$. If $\sigma$ is sufficiently small, then a mean-preserving spread on $\pi_{t}(\mu)$ increases the relative position in stocks of agents with risk-tolerance higher than one, and decreases it for those with risktolerance lower than one. That is

$$
\frac{\tilde{N}_{i t}^{\rho_{i}>1}}{\tilde{N}_{i t}^{\rho_{i}<1}}>\frac{N_{i t}^{\rho_{i}>1}}{N_{i t}^{\rho_{i}<1}}
$$

Hence, higher uncertainty on the drift rate of consumption increases the relative position in stocks of agents with risk tolerance higher than 1 and decreases it for agents with risk tolerance lower than 1 . This implies that when heterogeneity of risk aversion encompasses agents with risk tolerance above and below one, there is a clear increase in the dispersion of stock positions, and hence of trading across agents. The proposition can only be shown when $\sigma$ is sufficiently small by a continuity argument, but simulations below show that it is rather general fact.

Corollary 13 and Proposition 15 show that an increase in uncertainty brings about an increase in stock prices and an increase in the dispersion of portfolio holdings, which can only be achieved through trading. Because my model with general priors implies that uncertainty may endogenously increase, we can expect that the price of the stock to increase and the trading volume increase at the same time. The reason is that higher macroeconomic uncertainty increases risk sharing opportunities across heterogeneous agents and hence it generates additional trading. Moreover, higher uncertainty also implies that believes fluctuate more wildly, generating trading volume. 


\section{A Calibration}

To calibrate the model we need to first return to the issue of solving the fixed-point problem in Equations (9) and (10), that is:

$$
e^{\delta_{t}}=E^{C S}\left[s_{i} \lambda\left(\rho_{i}\right) e^{\rho_{i} g\left(\delta_{t}\right)}\right] ; \quad \lambda\left(\rho_{i}\right)=\frac{E_{0}^{\mu, x, \tau}\left[e^{\delta_{\tau}-g\left(\delta_{\tau}\right)}\right]}{E_{0}^{\mu, x, \tau}\left[e^{(\rho-1) g\left(\delta_{\tau}\right)}\right]}
$$

For general endowment distributions $s_{i}$, the functional fixed point is hard to solve. I rather proceed as follows:

1. Let the endowment be given by

$$
s_{i}=s\left(\rho_{i}\right) \tilde{s}_{i} \quad \text { where } \quad \tilde{s}_{i}>0, E\left[\tilde{s}_{i}\right]=1, \operatorname{Cov}\left[\tilde{s}_{i}, \rho_{i}\right]=0
$$

2. Assume the product $s(\rho) \lambda(\rho)$ has the functional form

$$
s(\rho) \lambda(\rho)=\frac{e^{-\rho y}}{E^{C S}\left[e^{-\rho y}\right]}
$$

for some constant $y$;

3. Compute $g\left(\delta_{t}\right)$ by solving the equation

$$
e^{\delta_{t}}=E^{C S}\left[s(\rho) \lambda(\rho) e^{\rho g\left(\delta_{t}\right)}\right]=\frac{E^{C S}\left[e^{\rho\left(g\left(\delta_{t}\right)-y\right)}\right]}{E^{C S}\left[e^{-\rho y}\right]} ;
$$

4. Compute

$$
\lambda(\rho)=\frac{E_{0}^{\mu, x, \tau}\left[e^{\delta_{\tau}-g\left(\delta_{\tau}\right)}\right]}{E_{0}^{\mu, x, \tau}\left[e^{(\rho-1) g\left(\delta_{\tau}\right)}\right]}
$$

5. Compute the initial endowment

$$
s(\rho)=\frac{1}{\lambda(\rho)} \frac{e^{-\rho y}}{E^{C S}\left[e^{-\rho y}\right]}
$$

Some algebra shows that $E^{C S}[s(\rho)]=1$.

When $\mu / \sigma$ is high, $\lambda(\rho)$ is decreasing in $\rho$, which in turn makes $s(\rho)$ increasing in $\rho$. This is a problem as the procedure above naturally gives more endowment to high risk tolerant agents. However, by judiciously choosing the constant y I can "undo" the impact of $\lambda(\rho)$ on $s(\rho)$ and obtain initial distribution for $s(\rho)$ that is increasing or decreasing in $\rho$ (or Ushaped). Unfortunately, the simple methodology above to numerically solve the model does 
not allow for the case with $s(\rho)=1$ for all $\rho$. Still, the random component $\tilde{s}_{i}$ provides noise in the initial distribution to make the initial endowment look like a flat "cloud".

While the above strategy can be carried out for any distribution of $\rho$, it is useful to have closed-form formulas for the cross-sectional averages. I thus let risk tolerance be distributed according to a truncated normal density:

$$
\rho_{i} \sim \operatorname{Tr} N\left(\bar{\rho}, \sigma_{\rho}^{2} ; \rho_{L}, \rho_{H}\right)
$$

where $\operatorname{Tr} N\left(\bar{\rho}, \sigma_{\rho}^{2}, \rho_{L}, \rho_{H}\right)$ denotes the truncated normal distribution with range $\left(\rho_{L}, \rho_{H}\right)$. The equilibrium equation (49) then shows that $g_{t}=g\left(\delta_{t}\right)$ is the solution of:

$$
\delta_{t}=\widehat{\rho} g_{t}+\frac{1}{2} \sigma_{\rho}^{2} g_{t}^{2}+\log \left(\frac{\Phi\left(\rho_{H} ; \widehat{\rho}+g_{t} \sigma_{\rho}^{2}, \sigma_{\rho}^{2}\right)-\Phi\left(\rho_{L} ; \widehat{\rho}+g_{t} \sigma_{\rho}^{2}, \sigma_{\rho}^{2}\right)}{\Phi\left(\rho_{H} ; \widehat{\rho}, \sigma_{\rho}^{2}\right)-\Phi\left(\rho_{L} ; \widehat{\rho}, \sigma_{\rho}^{2}\right)}\right)
$$

where $\widehat{\rho}=\bar{\rho}-y \sigma_{\rho}^{2}$, and $\Phi(. ; a, b)$ denotes the normal CDF with mean $a$ and variance $b$. The solution of (50) is straightforward to obtain numerically. ${ }^{12}$

Finally, my characterization of many quantities, such as wealth or stock prices, in the form of expectations allows me to efficiently exploit Monte Carlo integration for the calculations of such quantities. Given $\phi>0$, it is straightforward to simulate maturity times $\tau=$ $-\log (U) / \phi$, where $U \sim$ Uniform $[0,1]$ as well as $x \sim N(0,1)$ and $\mu \sim \pi_{t}(\mu)$. Given a large number of simulations (with $\tau$ truncated at $T$ ), all the quantities in the form $E_{t}^{\mu, x, \tau}\left[K\left(\delta_{t+\tau}\right)\right]$ can be easily computed as the average of the simulated functions at $(\mu, x, \tau)$. In particular, there is no need to take any integral explicitly.

Table 1 reports parameter choices for an illustration of the model's implications. I set the average economic growth at $\mu=3 \%$, which is in line with the data. I instead choose the volatility of consumption growth at $\sigma=6 \%$, higher than the aggregate consumption volatility estimated by national accounts (around $1.5 \%$ ). I choose a high volatility for illustrative purposes, as resolving the equity premium puzzle is not the point of this paper. I assume the prior $\pi_{0}(\mu)$ is uniform between $\mu_{L}=0$ and $\mu_{H}=.06, \mu \sim U[0, .06]$. A positive support is reasonable as the prior is about long-term growth.

\footnotetext{
${ }^{12}$ Indeed, if $\sigma_{\rho}^{2}$ is small and the distribution is away from the boundaries, then equation (50) is well approximated by $\delta_{t}=\widehat{\rho} g_{t}+\frac{1}{2} \sigma_{\rho}^{2} g_{t}^{2}$ whose solution is

$$
\widetilde{g}\left(\delta_{t}\right)=\frac{\sqrt{\widehat{\rho}^{2}+2 \sigma_{\rho}^{2} \delta_{t}}-\widehat{\rho}}{\sigma_{\rho}^{2}}
$$

The positive root of the quadratic equation ensures the same set of properties of $g\left(\delta_{t}\right)$ in Proposition 1 . Xiorius and Zapatero (2007) provide a similar expression for their case with habit persistence preferences.
} 
Table 1: Parameters

\begin{tabular}{cccccccccccc}
\hline$\mu_{L}$ & $\mu_{H}$ & $\widehat{\mu}$ & $\sigma$ & $\phi$ & $\bar{\rho}$ & $\sigma_{\rho}$ & $\rho_{L}$ & $\rho_{H}$ & $E^{C S}[\rho]$ & $y$ & $T$ \\
0 & 0.06 & 0.03 & 0.06 & 0.02 & 0.02 & 1 & 0.05 & 10 & 0.847 & 4 & 100 \\
\hline
\end{tabular}

The range of risk tolerance is between $\rho_{L}=0.02$ to $\rho_{H}=10$ which correspond to risk aversion parameters between 50 and 0.01 . I choose $\bar{\rho}=\rho_{L}=0.02$ to obtain a positively skewed distribution of risk tolerance (as the mode is at $\bar{\rho}$.) Evidence from Kimball et al. (2014) suggests such a strongly positively skewed distribution. It also conforms with the fact that a large part of the population does not invest in stocks. Given the truncated normal distribution, the average risk tolerance is given by

$$
E^{C S}[\rho]=\bar{\rho}+\sigma_{\rho}^{2} \frac{\phi\left(\rho_{L} ; \bar{\rho}, \sigma_{\rho}^{2}\right)-\phi\left(\rho_{H} ; \bar{\rho}, \sigma_{\rho}^{2}\right)}{\Phi\left(\rho_{H} ; \bar{\rho}, \sigma_{\rho}^{2}\right)-\Phi\left(\rho_{L} ; \bar{\rho}, \sigma_{\rho}^{2}\right)}=0.847
$$

which corresponds to an average risk aversion of 1.1806. Above, $\phi(. ; a, b)$ denotes the pdf a normal distribution with mean $a$ and variance $b$.

Panel A in Figure 1 shows the distribution of risk tolerance under this parameterization. The distribution gives the vast majority of density to high risk averse agents. But it is strongly positively skewed. So, there are agents with very low risk aversion. The dotted vertical line denotes the average risk tolerance.

Panel B shows the value of the function $g\left(\delta_{t}\right)$ for different values of output $D_{t}=e_{t}^{\delta}$. The output ranges between 3 and over 6 . To give a sense of measure of the $x$-axis, given a growth rate $\mu=.03$, the economy takes 23 years to double in size. ${ }^{13}$

Panels $\mathrm{C}$ and D show the weighted average risk tolerance and the implied risk aversion, respectively. Focusing on Panel D, the effective aggregate risk aversion is around 1.8 at $D_{t}=$ 3. As the economy improves, the effective risk aversion declines, as shown in Proposition 1. By the time the output doubles, the risk aversion dropped close to the average risk aversion. As the economy keeps growing the effective risk aversion keeps dropping to finally converge to $1 / \rho_{H}=0.1$ in the long-run.

Figure 2 shows the impact of the decrease in effective risk aversion and an increase in uncertainty on asset prices. For comparison's purposes, I keep $T=100$ across different levels of output $D_{t}$. That is, although I often discuss the behavior of asset prices as the economy "grows", there is no temporal dimension implicit in this or the remaining figures.

\footnotetext{
${ }^{13}$ The formula is time $=\log ($ multiple $) / \mu$.
} 

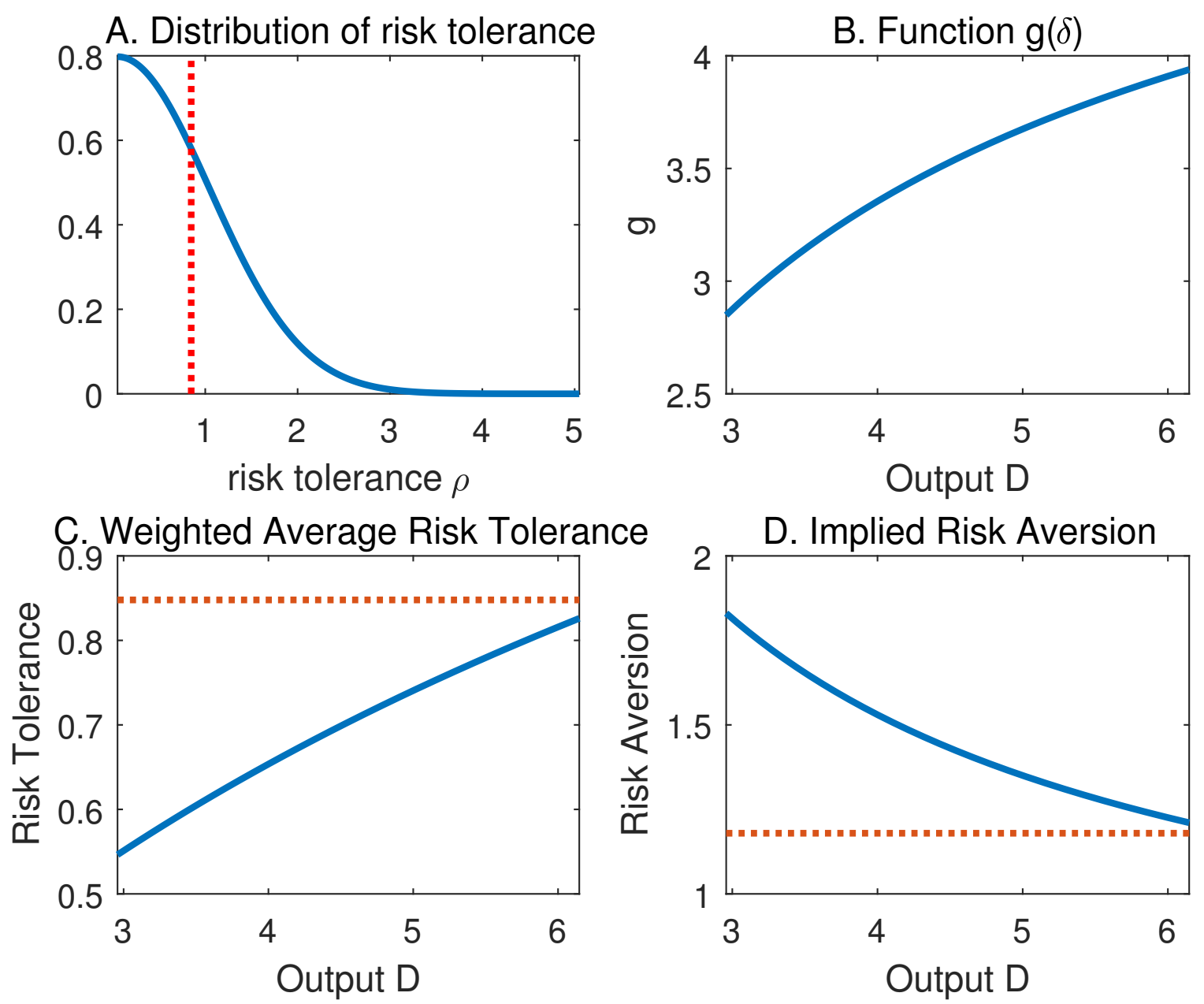

Figure 1: Risk Preferences: Real and Weighted. Panel A plots the distribution of risk tolerance used in the calibration. Panel B shows the function $g\left(\delta_{t}\right)$. Panel $\mathrm{C}$ plots the consumption weighted risk tolerance $E^{*}\left[\rho \mid \delta_{t}\right]$ and Panel D reports the implied risk aversion $\left(1 / E^{*}\left[\rho \mid \delta_{t}\right]\right)$. All parameters are in Table 1 .

Each panel of Figure 2 plots two lines. The solid line corresponds to the case with uncertainty, in which $\mu$ is uniformly distributed between $\mu_{L}=0$ and $\mu_{H}=0.06$ as in Table 1. The dashed line corresponds to the case of no uncertainty, with $\mu=3 \%$.

Panel A shows the $\mathrm{P} / \mathrm{D}$ ratio for $D$ ranging between 3 and 6 . In both cases of uncertainty and certainty, the $\mathrm{P} / \mathrm{D}$ ratio increases as the economy expands. Intuitively, as the effective risk aversion decreases - and especially the effective EIS increases - the $\mathrm{P} / \mathrm{D}$ increases. This result is consistent with our result in Proposition 4.

In addition, Panel $\mathrm{A}$ shows that higher uncertainty leads to a higher $\mathrm{P} / \mathrm{D}$ ratio, as the solid line is higher than the dashed line. This result is consistent with Corollary 13. Pastor and Veronesi (2006) exploits a similar argument in a model with habit preferences to highlight 

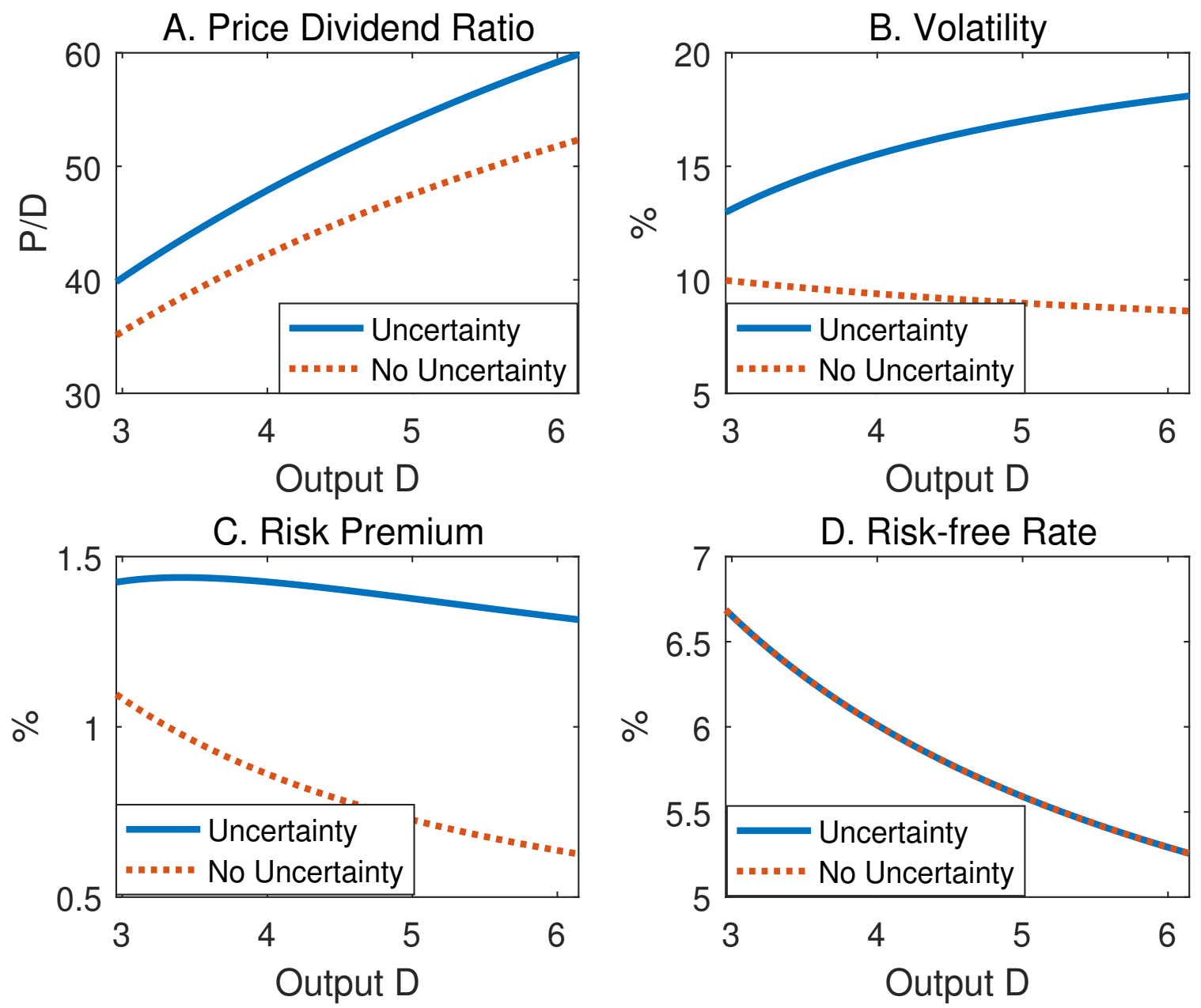

Figure 2: Asset Prices. Panel A plots the P/D ratio, Panel B the stock return volatility, Panel $\mathrm{C}$ the risk premium, and Panel $\mathrm{D}$ the risk-free rate. In each panel, the uncertainty case (solid line) assumes $\mu$ is uniformly distributed between 0 and $6 \%$, while the no-uncertainty case (dotted line) assumes $\mu=3 \%$. All other parameters are in Table 1.

that the high uncertainty about profitability of tech companies in the late 1990s may explain their high stock valuations at that time.

The high valuations in the late 1990s were accompanied by high volatility, which is also evident in this model by looking at Panel B. Uncertainty increases return volatility substantially from around $8 \%$ under certainty (dashed line) to around $13-18 \%$ under uncertainty (solid line). Hence, high valuations and high volatility go hand in hand when they are due to macroeconomic uncertainty.

Even in the case of certainty about $\mu$, the dotted line in Panel B shows that volatility is higher than fundamental volatility, which is at $6 \%$. This is due to the $\mathrm{P} / \mathrm{D}$ ratio being increasing in $\delta_{t}$, as discussed after Corollary 5 . 
Panel C plots the equity premium. Because the volatility of the stochastic discount factor is still low in the model, $\sigma_{M t}=\sigma / E_{t}^{*}[\rho]$, the equity premium is small, especially in the case of certainty, which ranges between just above $1 \%$ to $0.5 \%$. The risk premium declines in the case of certainty as the effective risk aversion declines as the economy expands. Under uncertainty, the risk premium is higher, around 1.5\%, but still far smaller than in the data (6\%). This model does not resolve the equity premium puzzle.

Finally, Panel D plots the risk-free rate, which is not affected by uncertainty. The risk free rate is high (the risk-free rate puzzle) and declines as the economy expands. Because the expected growth is high enough, the impact through the elasticity of intertemporal substitution dominates, as explained in Section 2.3.

In summary, the combination of learning and heterogeneity has a substantial impact on asset prices, as it increase prices, volatility, and the equity premium. We could expect that an (endogenous) increase in uncertainty thus leads to higher valuation, higher volatility, and a higher risk premium. Note that the positive relation between $\mathrm{P} / \mathrm{D}$ ratio and equity premium due to an increase in uncertainty would make standard predictive regressions fail. This is exactly what happened in the late 1990s, when the $\mathrm{P} / \mathrm{D}$ ratio lost its ability to predict future stock returns (see e.g. Menzly, Santos and Veronesi (2004)).

Figure 3 highlights the impact of uncertainty at the micro level. Panel A displays the dispersion of stock holdings across agents, computed as the standard deviation $\sqrt{V_{t}^{C S}\left[N_{i t}\right]}$. The plot shows that the dispersion of stock holdings is far higher under uncertainty (solid line) compared to the case of certainty (dotted line). As the economy expands the dispersion increases in both cases, but the difference due to uncertainty is dominating the figure. As explained, higher uncertainty generates additional risk-sharing motives, and households with high risk tolerance insure those with low risk tolerance.

Panels $\mathrm{C}$ and D show the distribution of positions in stocks and bonds, respectively, for the case in which output is $D_{t}=5$. The difference between the solid and dotted line shows that the dispersion of holdings is far larger for the case of uncertainty. Indeed, quite interestingly, very low risk tolerance households short the stock under uncertainty. This behavior is due to their hedging demands: because bad news about future output implies lower future consumption, such agents want to lower their consumption today and save more. By shorting the stock they gain from lower return and can purchase even more bonds to consume in the future. An extension of the model to allow for short-sale constraints would thus likely imply that such agents would not invest in stocks, as shown in the data. 

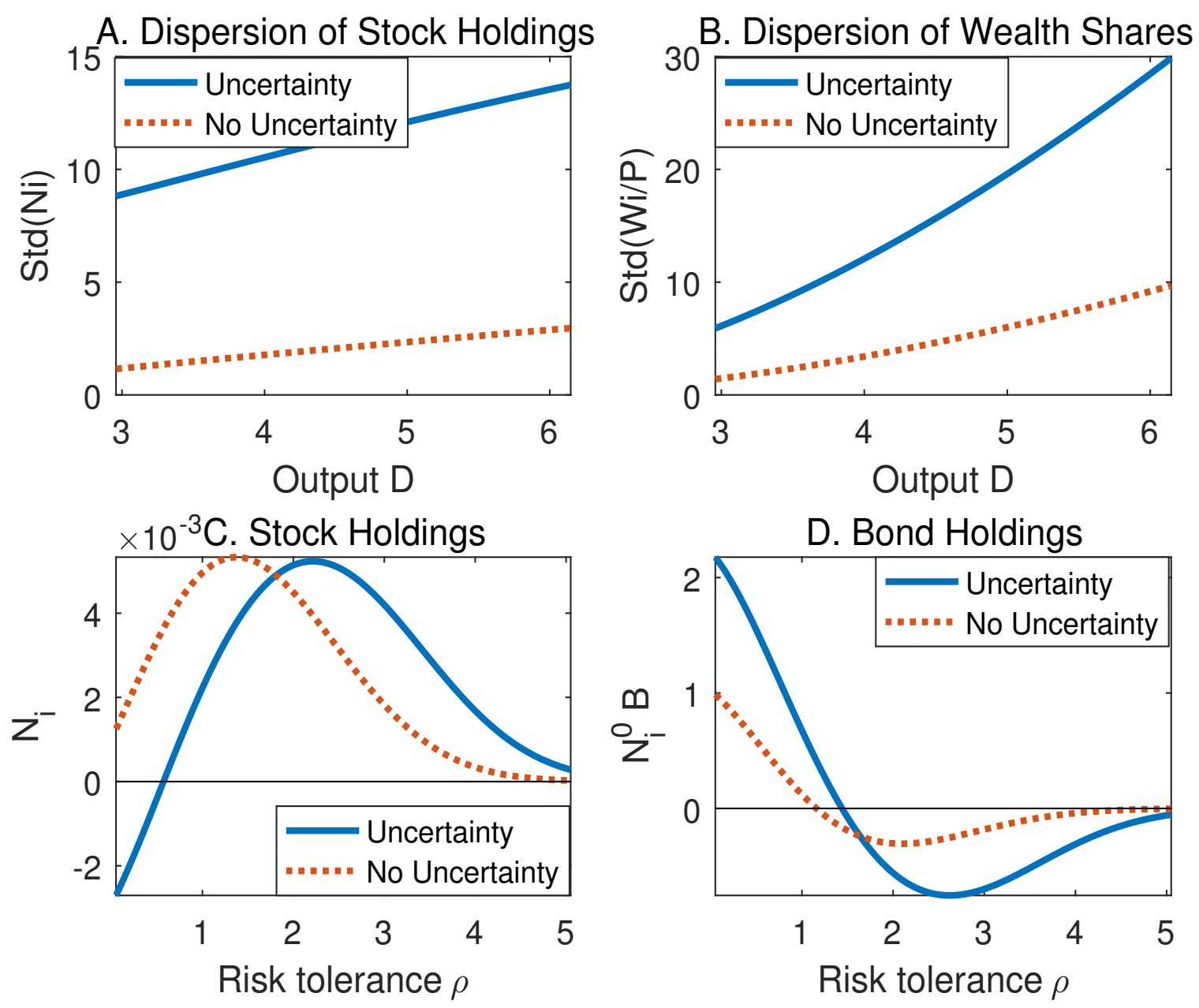

Figure 3: Portfolio Holdings and Wealth. Panel A plots the cross-sectional dispersion of stock holdings $\sqrt{V_{t}^{C S}\left[N_{i t}\right]}$. Panel B plots the cross-sectional dispersion of wealth across households. Panel $\mathrm{C}$ plots the actual distribution of stock holdings for the case $D=5$ across households, and panel D plots the distribution of bond holdings for the case $D=5$. In each panel, the uncertainty case (solid line) assumes $\mu$ is uniformly distributed between 0 and $6 \%$, while the no-uncertainty case (dotted line) assumes $\mu=3 \%$. All other parameters are in Table 1.

Finally, Panel B of Figure 3 shows that higher uncertainty about economic growth also implies a far higher dispersion of wealth shares across households. That is, a higher wealth inequality. The intuitive reason is that high uncertainty make households take on more extreme positions in stocks which in turn affect their wealth distribution.

The results of Figures 2 and 3 thus suggest that an increase in uncertainty should increase the $\mathrm{P} / \mathrm{D}$ ratio, return volatility, wealth inequality, and the dispersion of stock holdings, i.e. increase trading volume. These patterns are shown in Figure 4, which plots an example simulation with endogenous increase in uncertainty. Indeed, because I do not impose that 

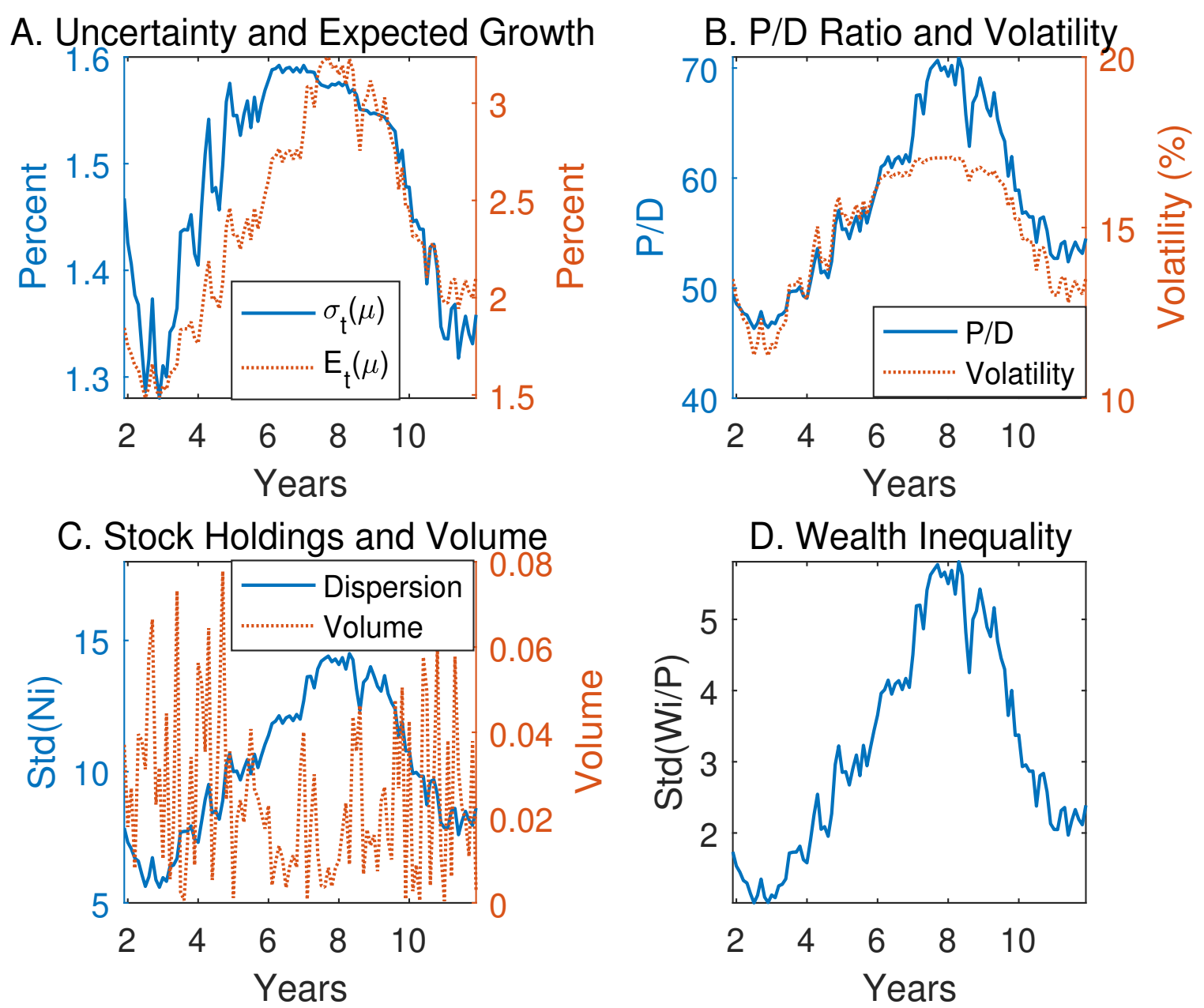

Figure 4: Endogenous Uncertainty, Asset Prices, and Portfolio Holdings. Panel A plots the uncertainty on economic growth (solid line) and expected growth (dotted line) in a simulated path. Uncertainty is defined as the standard deviation on economic growth $U_{t}=\sqrt{E_{t}\left[\left(\mu-E_{t}[\mu]\right)^{2}\right]}$. Panel B plots the corresponding $\mathrm{P} / \mathrm{D}$ ratio (solid line) and the stock return volatility (dotted line). Panel $\mathrm{C}$ plots the cross-sectional dispersion of stock holdings $\sqrt{V_{t}^{C S}\left[N_{i t}\right]}$ (solid line) and trading volume (dotted line), computed as the average absolute change in stock holdings. Panel D plots wealth inequality, computed as the cross-sectional dispersion in wealth shares. Parameters are in Table 1.

priors are normally distributed, the learning dynamics is non-linear. Although uncertainty decreases on average (eventually it will go to zero), there are episodes of endogenous increases in uncertainty due to realizations of shocks that are farther away from the mean.

Panel A of Figure 4 plots an example of an increase in uncertainty in simulation, where I compute uncertainty as the standard deviation of beliefs over $\mu$, that is $U_{t}=\sqrt{E_{t}\left[\left(\mu-E_{t}[\mu]\right)^{2}\right]}$. I purposely choose a time period in which such uncertainty increases, as it is evident between $t=3$ and $t=6$, when $U_{t}$ increases from $1.3 \%$ to $1.6 \%$. The panel also shows that at the 
same time expected economic growth $E_{t}[\mu]$ also increases from around $1.5 \%$ to $3 \%$.

The solid line in Panel $\mathrm{B}$ shows that the $\mathrm{P} / \mathrm{D}$ ratio increases substantially, from 48 to 70 , in response to such increase in expected growth and uncertainty. The large increase in the price valuation and the subsequent decline may be dubbed "a bubble" on an ex-post basis. The dotted line in the same panel shows that stock return volatility also increases in response to an increase in uncertainty, moving from $11 \%$ to $17 \%$.

The solid line in panel $\mathrm{C}$ shows that the dispersion of stock holdings across agents increased from 6 to 14, consistently with Proposition 15. As uncertainty and expected growth increases (Panel A) the dispersion also increases generating trading volume, as more risk tolerant agents purchase stocks from less risk tolerant agents. The dotted line in Panel C plots the trading volume, which is computed as the average absolute change in stock holdings at discrete time intervals. By comparing the dotted line with Panel A, we see that changes in both uncertainty and expected growth are accompanied by spikes in trading volume. Indeed, a regression of trading volume on absolute changes in uncertainty and expected growth yields a $90 \% R^{2}$, with both uncertainty and expected growth strongly significant.

Finally, Panel D plots the wealth inequality across agents, measured as the cross-sectional variance of wealth shares. The increase in uncertainty and expected growth generates a large increase of wealth inequality, as the increase in stock market make some households, the more risk tolerant, far more wealthier than others, the less risk tolerant.

To further emphasize the relation between the variation in uncertainty, the variation in economic growth, and trading volume, Figure 5 shows the scatterplots of trading volume (y-axis) against absolute changes in uncertainty (Panel A) and absolute changes in expected growth (Panel B). The scatterplots are computed over 50 simulated paths of 50-years each using the parameters in Table 1. As can be seen, variation in uncertainty explains $65 \%$ of trading volume, while variation in expected growth explains $74 \%$ of the trading volume. When combined, changes in both uncertainty and expected growth have a $R^{2}=84 \%$, with both quantities strongly significant. In summary, learning dynamics generates a large amount of trading volume in periods characterized by large changes in uncertainty and expected growth simply due to risk sharing motives.

The patterns of price movements, volatility, portfolio holdings, and trading volume depicted in panels $\mathrm{A}$ through $\mathrm{C}$ of Figure 4 are normally ascribed to bubbles and behavioral biases (see e.g. Scheinkman and Xiong (2003), Greenwood and Nagel (2009)) but as can be seen they can be generated in a model with heterogeneous agents and uncertainty about 

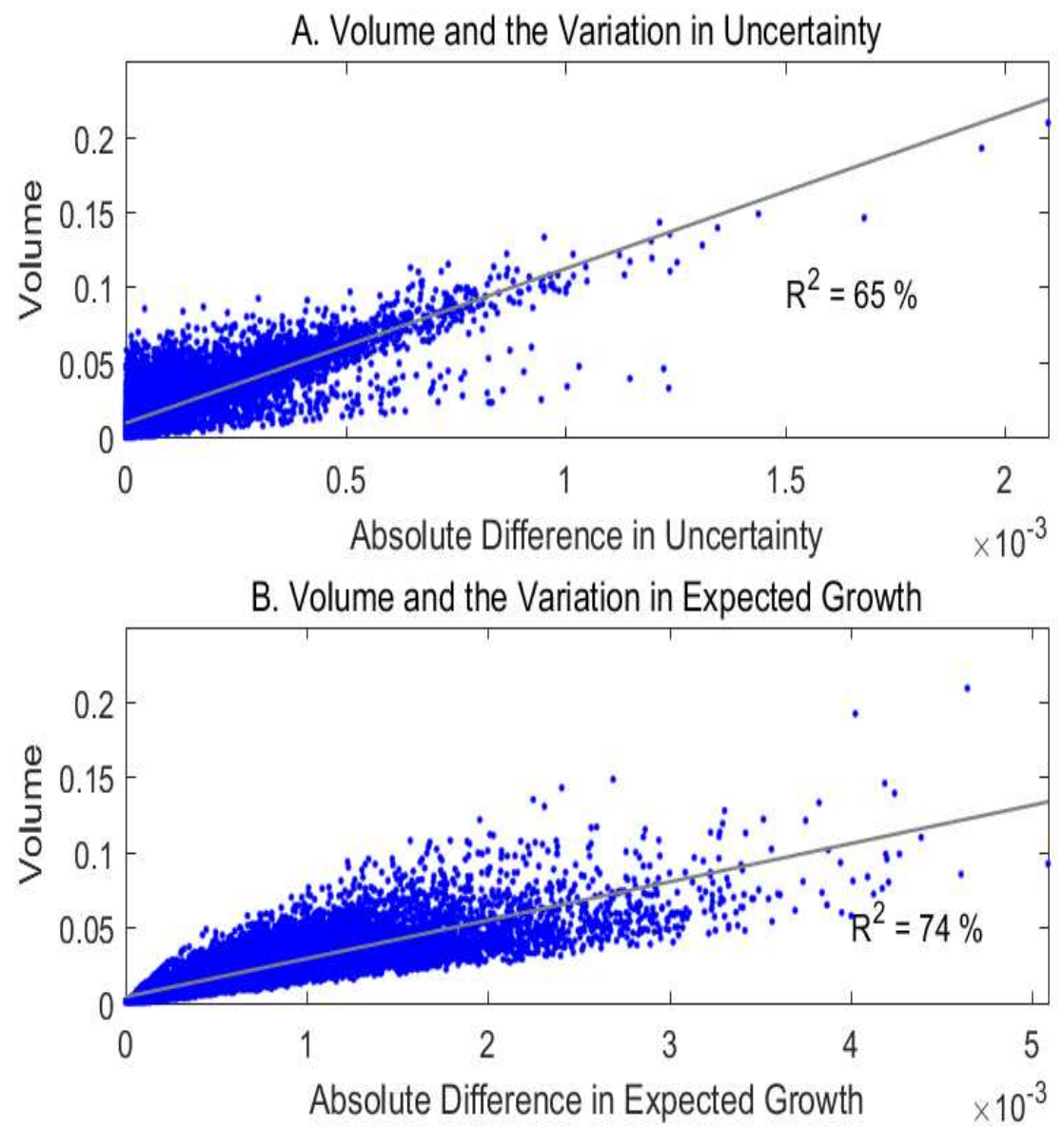

Figure 5: Trading Volume. Panel A plots the trading volume (y-axis) against the absolute changes in uncertainty (x-axis) on 50 simulated paths of 50 years each. The solid line represents the pooled regression line with its corresponding $R^{2}$. Panel B plots trading volume against absolute changes in expected aggregate growth. Trading volume is computed as the average absolute change in households' stock holdings at discrete intervals. Parameters are in Table 1.

long-term growth. ${ }^{14}$ The fact that the late 1990s were characterized by increasing uncertainty about long-term growth is quite plausible (see Pastor and Veronesi (2006) for discussion). While models with representative agents do not have predictions about portfolio holdings and trading, the contribution of this paper in this regard is to show that the same forces that generate higher price valuations and volatility also generate an increase in dispersion of portfolio holdings, and hence trading volume, due to higher need for risk sharing. In addi-

\footnotetext{
${ }^{14}$ Panel $\mathrm{C}$ of Figure 4 shows that trading volume tames at the peak of the "bubble" in this simulation, as uncertainty and economic growth also flatten (Panel A). Evidence suggests that trading volume was high also at the peak of the 1990s dot-com bubble, suggesting large variations in uncertainty and economic growth also at the peak, which the simulation in Figure 4 does not capture.
} 


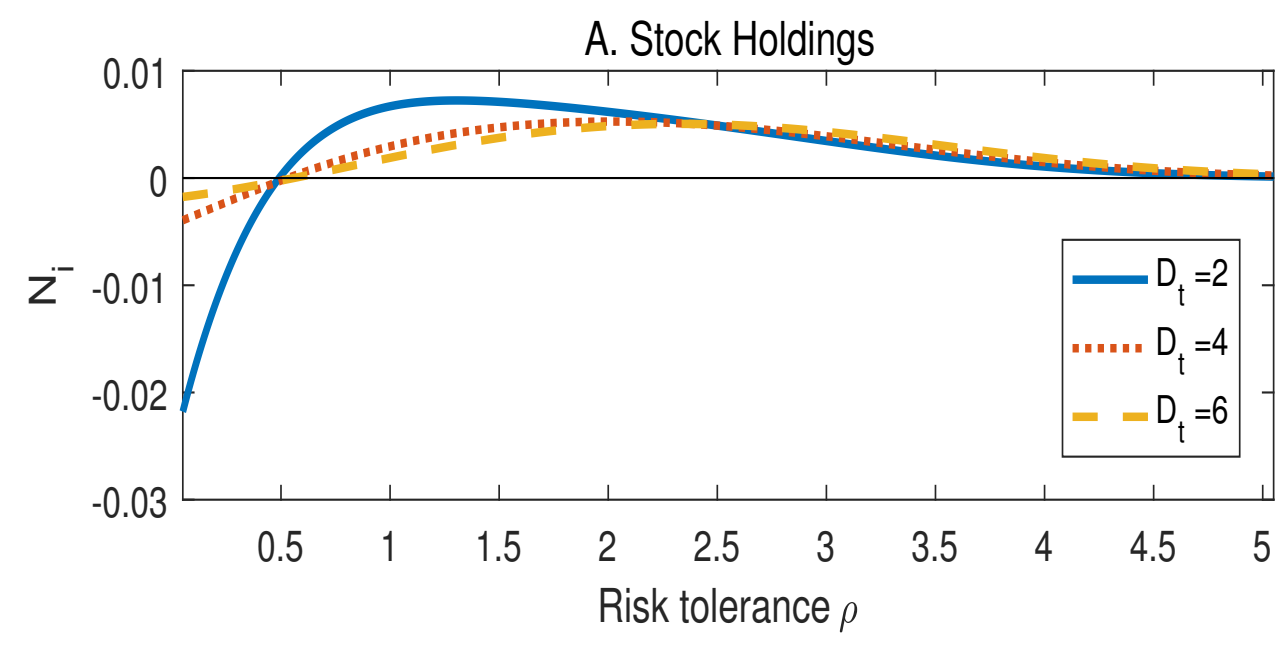

B. Bond Holdings

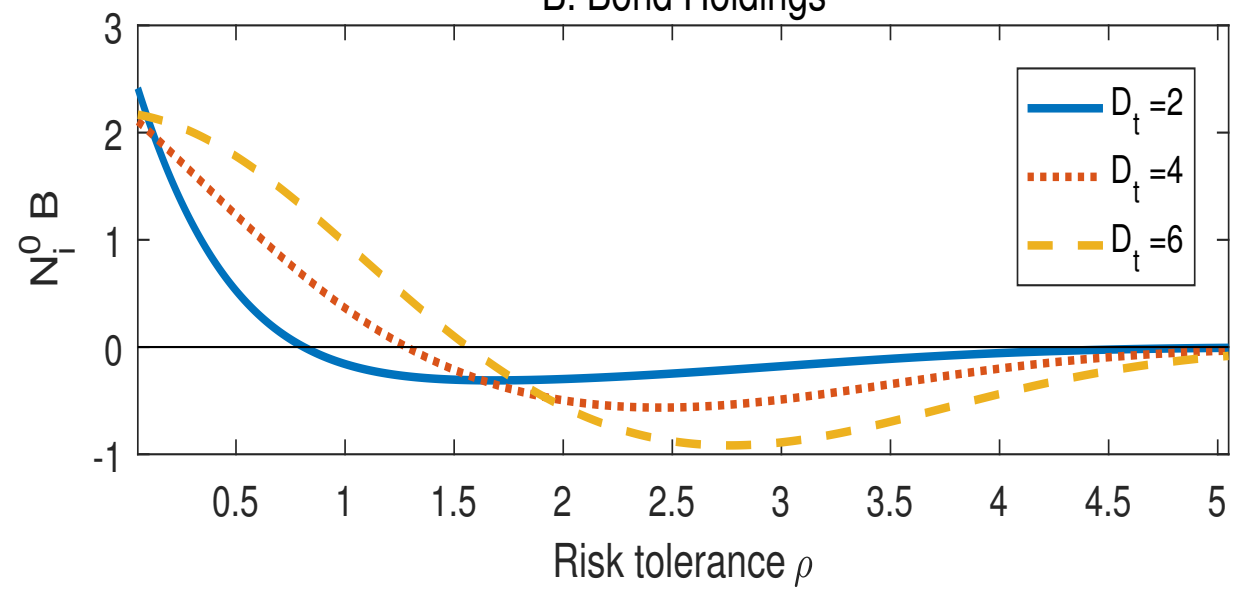

Figure 6: Portfolio Holdings. Panel A plots the distribution of stock holdings for three values of output $D_{t}=2,4,6$. Panel B plots the distribution of bond holdings for the same values. Parameters are in Table 1.

tion, wealth inequality also substantially increases due to the same forces, which is consistent with the trends in the late 1990s (see e.g. Saez and Zucman (2016)).

Finally, Figure 6 plots the distribution of stock holdings (Panel A) and bond holdings (Panel B) across households for three values of $D_{t}$. This figure highlights that households with intermediate risk tolerance qualitatively change their portfolio holdings as the economy expands. Consistently with the result of Proposition 10, households with risk aversion around 1 are borrowers for low $D_{t}=2$, but they become lenders for $D_{t}=6$, although at different times depending on risk tolerance. Households at the extremes, instead, are either always lenders (low risk tolerance) or always borrowers (high risk tolerance). The identity of borrowers and lenders thus change over time, as it depends on the risk-return characteristics of stock returns and the level of interest rates. 


\section{Conclusions}

The documented households heterogeneity in risk preferences and endowments is crucial to understand the dynamics of their behavior in response to a changing economic environment as well as to understand equilibrium asset prices. I show that such heterogeneity combined with time-varying uncertainty about long-term growth produces several empirical facts that are consistent with the empirical evidence. As the economy expands, the share of agents who do not keep up with the economy increases, the wealth distribution becomes more extreme, and households qualitatively modify their position in stocks and bonds. For instance, households with intermediate risk preferences move from being borrowers and leverage their purchases of risky assets to lenders and choose more conservative positions.

More important, the increase in macroeconomic uncertainty exacerbates heterogeneity across agents and provides further motives for risk sharing: With high uncertainty, households with high risk tolerance take on much more aggressive positions than households with low risk tolerance. Moreover, endogenous increases in uncertainty increase trading motives and hence yields additional trading volume as agents move to more extreme positions in risky stocks. My model predicts that an endogenous increase in uncertainty increases price valuations, return volatility, wealth inequality, and dispersion in traders' portfolio holdings, consistently with the evidence during the dot-com bubble of the late 1990s.

The model is set in the convenient framework of complete markets. Under learning, the model implies that households with low risk tolerance may hold short positions in stocks. Introducing incomplete markets and short sale constraints in my model with heterogeneous agents and learning may thus yield the result that the most risk averse agents do not invest in the stock market, consistently with the data. Time varying uncertainty and expected growth would still lead to large variation in trading volume because of the large number of other agents who are not constrained. Unfortunately, the case with incomplete markets is substantially more challenging and I thus leave this topic to future research. 


\section{References}

Ai, Hengjie (2010) "Information Quality and Long-run Risk: Asset Pricing Implications," Journal of Finance, vol. 65, Issue 4, 1333-1367

Barsky, Robert B., F. Thomas Juster Miles S. Kimball Matthew D. Shapiro (1997) "Preference Parameters and Behavioral Heterogeneity: An Experimental Approach in the Health and Retirement Study," Quarterly Journal of Economics, 112, 2, 537-579.

Bhamra, Horjat and Raman Uppal, (2014), "Asset Prices with Heterogeneity in Preferences and Beliefs," The Review of Financial Studies.

Brennan, Michael J., and Yihong Xia, (2001), "Stock return volatility and equity premium," Journal of Monetary Economics 47

Chan, Yeung Lewis and Leonid Kogan (2002) "Catching up with the Jonese: Heterogeneous Preferences and the Dynamics of Asset Prices," Journal of Political Economy, 110, no. 6: $1255-1285$.

Chiappori, P. A., Krislert Samphantharak, Sam Schulhofer-Wohl, and Robert M. Townesend, (2014) "Heterogeneity and risk sharing in village economies," Quantitative Economics, $1-27$.

Colling-Dufresne, Pierre, Michael Johannes, and Lars Lochster (2016) "Parameter learning in General Equilibrium: The Asset Pricing Implications," American Economic Review, 106 (3), 664-698.

Cox, John, and Chi-fu Huang (1989) "Optimal Consumption and Portfolio Policies when Asset Prices Follow a Diffusion Process." Journal of Economic Theory Vol. 49, No. 1: $33-893$.

Cvitanic, J., E. Jouini, S. Malamud and C. Napp (2012) "Financial Markets Equilibrium with Heterogeneous Agents." Review of Finance, 16, 285321.

David, Alexander (1997) "Fluctuating Confidence in Stock Markets: Implications for Returns and Volatility Journal of Financial and Quantitative Analysis.

David, Alexander and Pietro Veronesi (2013) "What Ties Return Volatilities to Price Valuations and Fundamentals?" Journal of Political Economy, 121, 4, 682-746.

Dumas, B. (1989) "Two-person dynamic equilibrium in the capital market," Review of Financial Studies, Vol. 2, pp. 157?188. 
Feldman, Mark, and Gilles, Christian. (1985) "An Expository Note on Individual Risk without Aggregate Uncertainty." J. Econ. Theory 35 (February): 2632

Gomez, Matthieu (2017) "Asset Prices and Wealth Inequality," working paper, Princeton University.

Greenwood R. and S. Nagel (2009) "Inexperienced Investors and the Bubble" Journal of Financial Economics, 93, 239-258.

Kimball Miles S, Claudia R. Sahm, and Matthew D Shapiro (2008) "Inputing Risk Tolerance from Survey Responses" Journal of the American Statistical Association, 103: 483, $1028-1038$

Judd, Kenneth L. (1985) "The Law of Large Numbers with a Continuum of IID Random Variables." J. Econ. Theory 35 (February): 19-25.

Liptser Robert S. and N. Shiryaev (2001) "Statistics of Random Processes" Springer-Verlag Berlin Heidelberg .

Longstaff, Francis A. and Jiang Wang (2012) "Asset Pricing and the Credit Market," Review of Financial Studies, vol. 25 (11): 3169-3215.

Menzly, Lior, Tano Santos, and Pietro Veronesi (2004) "Understanding Predictability" Journal of Political Economy, 112, 1: 1-47.

Pastor, Lubos and Pietro Veronesi (2006) "Was There a NASDAQ Bubble in the Late 1990s?" Journal of Financial Economics 81, 61 - 100

Pastor, Lubos and Pietro Veronesi (2018) "Inequality Aversion, Populism, and the Backlash Against Globalization," Working Paper, University of Chicago.

Saez, Emmanuel and Gabriel Zucman (2016) "Wealth Inequality in the United States since 1913: Evidence from Capitalized Income Tax Data," Quarterly Journal of Economics, vol. 131 no. 2, 519-578.

Santos, Tano and Pietro Veronesi (2018) "Leverage" Working Paper, University of Chicago.

Scheinkman, Jose A. (2014) "Speculation, Trading, and Bubbles," Kenneth J. Arrow Lecture Series, Columbia University Press, New York.

Scheinkman, Jose A. and Wei Xiong (2003) "Overconfidence and Speculative Bubbles," Journal of Political Economy, December 2003, 1183-1219. 
Townsend, Robert M. "Risk and Insurance in Village india," Econometrica, May, 62(3), $539-591$.

Veronesi, Pietro (1999) "Stock Market Overreaction to Bad News in Good Times: A Rational Expectations Equilibrium Model," Review of Financial Studies , 12, 5.

Veronesi, Pietro (2000) "How does Information Quality Affect Stock Returns?" Journal of Finance, 55, 2.

Wang, Jiang (1996) "The Term Structure of Interest Rates in a Pure Exchange Economy with Heterogeneous Investors," Journal Financial Economics 41 (May): 75-110.

Xiouros, C. and F. Zapatero, (2010) "The Representative Agent of an Economy with External Habit-Formation and Heterogeneous Risk-Aversion," Review of Financial Studies, 23: $3017-3047$. 


\section{Appendix: Proofs}

Proof of Proposition 1. The equilibrium condition defines $g\left(\delta_{t}\right)$ as the solution to

$$
\delta_{t}=\log \left(E\left[s_{i} \lambda\left(\rho_{i}\right) e^{\rho_{i} g}\right]\right)
$$

The right-hand-side (RHS) of this equation is monotonically increasing in $g$, as

$$
\frac{\partial \log \left(E\left[s_{i} \lambda\left(\rho_{i}\right) e^{\rho_{i} g}\right]\right)}{\partial g}=\frac{E\left[s_{i} \rho_{i} \lambda\left(\rho_{i}\right) e^{\rho_{i} g}\right]}{E\left[s_{i} \lambda\left(\rho_{i}\right) e^{\rho_{i} g}\right]}>0 .
$$

Thus the solution $g\left(\delta_{t}\right)$ is unique. Note that we have the restriction $M_{0}=1$ and assuming $\delta_{0}=0$ without loss of generality, the restriction requires the following normalization

$$
0=\log \left(E\left[s_{i} \lambda\left(\rho_{i}\right)\right]\right)
$$

Take now the total differential with respect to $\delta_{t}$ to find

$$
1=\frac{g^{\prime}\left(\delta_{t}\right) E\left[\rho_{i} s_{i} \lambda\left(\rho_{i}\right) e^{\rho_{i} g\left(\delta_{t}\right)}\right]}{E\left[s_{i} \lambda\left(\rho_{i}\right) e^{\rho_{i} g\left(\delta_{t}\right)}\right]}
$$

Define a new density over $\rho_{i}$

$$
f^{*}\left(\rho_{i} \mid \delta_{t}\right)=\frac{\int f\left(\rho_{i}, s_{i}\right) s_{i} \lambda\left(\rho_{i}\right) e^{\rho_{i} g\left(\delta_{t}\right)} d s_{i}}{\iint f\left(\rho_{i}, s_{i}\right) s_{i} \lambda\left(\rho_{i}\right) e^{\rho_{i} g\left(\delta_{t}\right)} d \rho_{i} d s_{i}}
$$

Thus obtaining

$$
g^{\prime}\left(\delta_{t}\right)=\frac{1}{E^{*}\left[\rho \mid \delta_{t}\right]}>0
$$

That is, $g\left(\delta_{t}\right)$ is globally increasing, and $g\left(\delta_{t}\right)>0$ for $\delta_{t}>0$.

To prove concavity, rewrite

$$
g^{\prime}\left(\delta_{t}\right)=\frac{E\left[e^{-\rho_{i} \log \left(\xi_{i}\right)+\rho_{i} g\left(\delta_{t}\right)}\right]}{E\left[\rho_{i} e^{-\rho_{i} \log \left(\xi_{i}\right)+\rho_{i} g\left(\delta_{t}\right)}\right]}
$$

and compute the second derivative

$$
\begin{aligned}
g^{\prime \prime}\left(\delta_{t}\right) & \left.=\frac{E\left[\rho_{i} g^{\prime}\left(\delta_{t}\right) e^{-\rho_{i} \log \left(\xi_{i}\right)+\rho_{i} g\left(\delta_{t}\right)}\right]}{E\left[\rho_{i} e^{-\rho_{i} \log \left(\xi_{i}\right)+\rho_{i} g\left(\delta_{t}\right)}\right]}-\frac{E\left[e^{-\rho_{i} \log \left(\xi_{i}\right)+\rho_{i} g\left(\delta_{t}\right)}\right] E\left[\rho_{i}^{2} g^{\prime}\left(\delta_{t}\right) e^{-\rho_{i} \log \left(\xi_{i}\right)+\rho_{i} g\left(\delta_{t}\right)}\right]}{\left(E\left[\rho_{i} e^{-\rho_{i} \log \left(\xi_{i}\right)+\rho_{i} g\left(\delta_{t}\right)}\right]\right)^{2}}\right\} \\
& =g^{\prime}\left(\delta_{t}\right)\left\{\frac{E\left[\rho_{i} e^{-\rho_{i} \log \left(\xi_{i}\right)+\rho_{i} g\left(\delta_{t}\right)}\right]}{E\left[\rho_{i} e^{-\rho_{i} \log \left(\xi_{i}\right)+\rho_{i} g\left(\delta_{t}\right)}\right]}-\frac{E\left[e^{-\rho_{i} \log \left(\xi_{i}\right)+\rho_{i} g\left(\delta_{t}\right)}\right] E\left[\rho_{i}^{2} e^{-\rho_{i} \log \left(\xi_{i}\right)+\rho_{i} g\left(\delta_{t}\right)}\right]}{\left(E\left[\rho_{i} e^{-\rho_{i} \log \left(\xi_{i}\right)+\rho_{i} g\left(\delta_{t}\right)}\right]\right)^{2}}\right\} \\
& =g^{\prime}\left(\delta_{t}\right)\left\{1-\frac{E\left[e^{-\rho_{i} \log \left(\xi_{i}\right)+\rho_{i} g\left(\delta_{t}\right)}\right] E\left[\rho_{i}^{2} e^{-\rho_{i} \log \left(\xi_{i}\right)+\rho_{i} g\left(\delta_{t}\right)}\right]}{E\left[\rho_{i} e^{-\rho_{i} \log \left(\xi_{i}\right)+\rho_{i} g\left(\delta_{t}\right)}\right] E\left[\rho_{i} e^{-\rho_{i} \log \left(\xi_{i}\right)+\rho_{i} g\left(\delta_{t}\right)}\right]}\right\} \\
& =g^{\prime}\left(\delta_{t}\right)\left\{1-\frac{E^{* *}\left[\rho_{i} \mid \delta_{t}\right]}{E^{*}\left[\rho_{i} \mid \delta_{t}\right]}\right\}
\end{aligned}
$$


where now

$$
\begin{aligned}
f^{* *}\left(\rho \mid \delta_{t}\right) & =\frac{\rho \int f(\rho, \xi) e^{-\rho_{i} \log \left(\xi_{i}\right)+\rho_{i} g\left(\delta_{t}\right)} d \xi}{\iint f(\rho, \xi) \rho e^{-\rho_{i} \log \left(\xi_{i}\right)+\rho_{i} g\left(\delta_{t}\right)} d \xi d \rho} \\
& =\frac{\rho f^{*}\left(\rho \mid \delta_{t}\right) \iint e^{-\rho_{i} \log \left(\xi_{i}\right)+\rho_{i} g\left(\delta_{t}\right)} d \rho_{i} d \xi_{i}}{\iint f(\rho, \xi) \rho e^{-\rho_{i} \log \left(\xi_{i}\right)+\rho_{i} g\left(\delta_{t}\right)} d \xi d \rho} \\
& =\frac{\rho f^{*}\left(\rho \mid \delta_{t}\right)}{\int \rho f^{*}\left(\rho \mid \delta_{t}\right) d \rho}
\end{aligned}
$$

Note that $f^{* *}\left(\rho \mid \delta_{t}\right)$ provides uniformly higher mass to higher $\rho$ than lower $\rho$, and thus the expectation using $f^{* *}\left(\rho \mid \delta_{t}\right)$ must be higher than using $f^{*}\left(\rho \mid \delta_{t}\right)$. Hence

$$
E^{* *}\left[\rho \mid \delta_{t}\right]>E^{*}\left[\rho \mid \delta_{t}\right]
$$

which gives

$$
g^{\prime \prime}\left(\delta_{t}\right)=g^{\prime}\left(\delta_{t}\right)\left\{1-\frac{E^{* *}\left[\rho_{i} \mid \delta_{t}\right]}{E^{*}\left[\rho_{i} \mid \delta_{t}\right]}\right\}<0
$$

Finally, as $\delta_{t}$ increases and thus $g\left(\delta_{t}\right)>0$, the density

$$
f^{*}\left(\rho_{i} \mid \delta_{t}\right)=\frac{\int f\left(\rho_{i}, \xi_{i}\right) e^{-\rho_{i} \log \left(\xi_{i}\right)+\rho_{i} g\left(\delta_{t}\right)} d \xi_{i}}{\iint e^{-\rho_{i} \log \left(\xi_{i}\right)+\rho_{i} g\left(\delta_{t}\right)} d \rho_{i} d \xi_{i}}
$$

gives increasingly higher weight to high $\rho_{i}$ (recall that $\xi_{i}$ are fixed constants) and therefore $f^{*}\left(\rho \mid \delta_{t}\right)$ converges to a degenerate distribution around $\rho_{H}$ and hence

$$
g^{\prime}\left(\delta_{t}\right) \rightarrow \frac{1}{\rho_{H}}
$$

Similarly, as $\delta_{t}$ decreases, $g(\delta)<0$ and the distribution $f^{*}\left(\rho \mid \delta_{t}\right)$ becomes degenerate around $\rho_{L}$.

Q.E.D.

Proof of Proposition 2. We know that the representative agent is given by

$$
\mathcal{U}\left(D_{t}, t\right)=\max _{\left\{C_{i t}\right\}} \int \frac{1}{\xi_{i}} \frac{e^{-\phi t} C_{i t}^{1-\frac{1}{\rho_{i}}}}{1-\frac{1}{\rho_{i}}} d i \text { subject to } \int C_{i t} d i=D_{t}
$$

Therefore, we have from the FOC of the social planner Lagrangean that

$$
\frac{1}{\xi_{i}} e^{-\phi t} C_{i t}^{-\frac{1}{\rho_{i}}}=M_{t}
$$

where $M_{t}$ is the lagrange multiplier (where we use the notation in the decentralized economy, i.e. $M_{t}$ is the state price density). Therefore, we have the solution for consumption is the 
one discussed in the text. The utility function of the representative agent is thus

$$
\begin{aligned}
\mathcal{U}\left(D_{t}, t\right) & =e^{-\phi t} \int e^{-\log (\xi)} \frac{\left(e^{-\rho_{i} \log (\xi)+\rho_{i} g\left(\delta_{t}\right)}\right)^{1-\frac{1}{\rho_{i}}}}{1-\frac{1}{\rho_{i}}} d i \\
& =e^{-\phi t} \int \frac{e^{-\rho_{i} \log (\xi)+\left(\rho_{i}-1\right) g\left(\delta_{t}\right)}}{1-\frac{1}{\rho_{i}}} d i
\end{aligned}
$$

Recall

$$
e^{-\rho_{i} \log (\xi)}=s_{i} \lambda\left(\rho_{i}\right)
$$

thus

$$
\mathcal{U}\left(D_{t}, t\right)=e^{-\phi t} \int \frac{s_{i} \lambda\left(\rho_{i}\right) e^{\left(\rho_{i}-1\right) g(\log (D))}}{1-\frac{1}{\rho_{i}}} d i
$$

We now compute the risk aversion

$$
R A(D)=-\frac{D \mathcal{U}^{\prime \prime}(D, t)}{\mathcal{U}^{\prime}(D, t)}
$$

We have

$$
\begin{aligned}
\mathcal{U}^{\prime}\left(D_{t}, t\right)= & e^{-\phi t} \int \frac{s_{i} \lambda\left(\rho_{i}\right) e^{\left(\rho_{i}-1\right) g(\log (D))}\left(\rho_{i}-1\right) g^{\prime}(\delta)}{1-\frac{1}{\rho_{i}}} d i \frac{1}{D} \\
= & e^{-\phi t} \int \frac{s_{i} \lambda\left(\rho_{i}\right) e^{\left(\rho_{i}-1\right) g(\log (D))} \rho_{i}\left(\rho_{i}-1\right) g^{\prime}(\delta)}{\left(\rho_{i}-1\right)} d i \frac{1}{D} \\
= & g^{\prime}(\log (D)) e^{-\phi t} \int s_{i} \lambda\left(\rho_{i}\right) e^{\left(\rho_{i}-1\right) g(\log (D))} \rho_{i} d i \frac{1}{D} \\
\mathcal{U}^{\prime \prime}(D, t)= & g^{\prime \prime}(\log (D)) \frac{1}{D} e^{-\phi t} \int s_{i} \lambda\left(\rho_{i}\right) e^{\left(\rho_{i}-1\right) g(\log (D))} \rho_{i} d i \frac{1}{D} \\
& +g^{\prime}(\log (D)) e^{-\phi t} \int s_{i} \lambda\left(\rho_{i}\right) e^{\left(\rho_{i}-1\right) g(\log (D))}\left(\rho_{i}-1\right) \rho_{i} g^{\prime}(\log (D)) \frac{1}{D} d i \frac{1}{D} \\
& -g^{\prime}(\log (D)) e^{-\phi t} \int s_{i} \lambda\left(\rho_{i}\right) e^{\left(\rho_{i}-1\right) g(\log (D))} \rho_{i} d i \frac{1}{D^{2}}
\end{aligned}
$$

or

$$
\begin{aligned}
\mathcal{U} "(D, t)= & g "(\log (D)) \frac{1}{D^{2}} e^{-\phi t} \int s_{i} \lambda\left(\rho_{i}\right) e^{\left(\rho_{i}-1\right) g(\log (D))} \rho_{i} d i \\
& +g^{\prime}(\log (D))^{2} \frac{1}{D^{2}} e^{-\phi t} \int s_{i} \lambda\left(\rho_{i}\right) e^{\left(\rho_{i}-1\right) g(\log (D))}\left(\rho_{i}-1\right) \rho_{i} d i \\
& -g^{\prime}(\log (D)) e^{-\phi t} \int s_{i} \lambda\left(\rho_{i}\right) e^{\left(\rho_{i}-1\right) g(\log (D))} \rho_{i} d i \frac{1}{D^{2}}
\end{aligned}
$$

Therefore, recall

$$
R A(D)=-\frac{D \mathcal{U}^{\prime \prime}(D, t)}{\mathcal{U}^{\prime}(D, t)}
$$




$$
\begin{aligned}
& =-\left[\begin{array}{c}
\frac{g^{\prime \prime}(\log (D)) \int s_{i} \lambda\left(\rho_{i}\right) e^{\left(\rho_{i}-1\right) g(\log (D))} \rho_{i} d i}{g^{\prime}(\log (D)) \int s_{i} \lambda\left(\rho_{i}\right) e^{\left(\rho_{i}-1\right) g(\log (D))} \rho_{i} d i}+\frac{g^{\prime}(\log (D))^{2} \int s_{i} \lambda\left(\rho_{i}\right) e^{\left(\rho_{i}-1\right) g(\log (D))}\left(\rho_{i}-1\right) \rho_{i} d i}{g^{\prime}(\log (D)) \int s_{i} \lambda\left(\rho_{i}\right) e^{\left(\rho_{i}-1\right) g(\log (D))} \rho_{i} d i} \\
-\frac{g^{\prime}(\log (D)) \int s_{i} \lambda\left(\rho_{i}\right) e^{\left(\rho_{i}-1\right) g(\log (D))} \rho_{i} d i}{g^{\prime}(\log (D)) \int s_{i} \lambda\left(\rho_{i}\right) e^{\left(\rho_{i}-1\right) g(\log (D))} \rho_{i} d i}
\end{array}\right] \\
& =-\left[\frac{g^{\prime \prime}(\log (D))}{g^{\prime}(\log (D))}+\frac{g^{\prime}(\log (D)) \int s_{i} \lambda\left(\rho_{i}\right) e^{\left(\rho_{i}-1\right) g(\log (D))} \rho_{i}^{2} d i}{\int s_{i} \lambda\left(\rho_{i}\right) e^{\left(\rho_{i}-1\right) g(\log (D))} \rho_{i} d i}-g^{\prime}(\log (D))-1\right] \\
& =-\left[\frac{g^{\prime \prime}(\log (D))}{g^{\prime}(\log (D))}+g^{\prime}(\log (D)) E^{* *}[\rho]-g^{\prime}(\log (D))-1\right]
\end{aligned}
$$

Recall now

$$
\begin{aligned}
g^{\prime}(\delta) & =\frac{1}{E^{*}[\rho \mid \delta]} \\
g^{\prime \prime}(\delta) & =g^{\prime}(\delta)\left[1-\frac{\left.E^{* *}[\rho \mid \delta]\right]}{E^{*}[\rho \mid \delta]}\right]
\end{aligned}
$$

obtaining

$$
\begin{aligned}
R A(D) & =-\left[\frac{g^{\prime}(\delta)}{g^{\prime}(\log (D))}\left[1-\frac{E^{* *}[\rho \mid \delta]}{E^{*}[\rho \mid \delta]}\right]+g^{\prime}(\log (D)) E^{* *}[\rho]-g^{\prime}(\log (D))-1\right] \\
& =-\left[1-g^{\prime}(\log (D)) E^{* *}[\rho \mid \delta]+g^{\prime}(\log (D)) E^{* *}[\rho]-g^{\prime}(\log (D))-1\right] \\
& =g^{\prime}(\log (D)) \\
& =g^{\prime}\left(\delta_{t}\right)
\end{aligned}
$$

Q.E.D.

Proof of Corollary 2. From (5) and (9) the consumption share is

$$
\frac{C_{i t}}{D_{t}}=e^{-\rho_{i} \log \left(\xi_{i}\right)+\rho_{i} g\left(\delta_{t}\right)-\delta_{t}}
$$

Therefore

$$
\frac{\partial C_{i t} / D_{t}}{\partial \delta_{t}}=\left(\rho_{i} g^{\prime}\left(\delta_{t}\right)-1\right) e^{-\rho_{i} \log \left(\xi_{i}\right)+\rho_{i} g\left(\delta_{t}\right)-\delta_{t}}>0
$$

if and only if

$$
\rho_{i} g^{\prime}\left(\delta_{t}\right)-1>0
$$

i.e. if and only if

$$
\rho_{i}>\frac{1}{g^{\prime}\left(\delta_{t}\right)}=E^{*}\left[\rho \mid \delta_{t}\right]
$$

The last point follows from the fact that $g^{\prime}\left(\delta_{t}\right)$ is decreasing and hence $E^{*}\left[\rho \mid \delta_{t}\right]$ is increasing in $\delta_{t}$. As the economy expands, fewer agents satisfy this condition. Q.E.D.

Proof of Corollary 3: From

$$
C_{i t}=e^{-\rho_{i} \log \left(\xi_{i}\right)+\rho_{i} g\left(\delta_{t}\right)}
$$


Ito's Lemma implies

$$
d C_{i t}=e^{-\rho_{i} \log \left(\xi_{i}\right)+\rho_{i} g\left(\delta_{t}\right)} \rho_{i} g^{\prime}(\delta) d \delta_{t}+\frac{1}{2} e^{-\rho_{i} \log \left(\xi_{i}\right)+\rho_{i} g\left(\delta_{t}\right)}\left\{\left[\rho_{i} g^{\prime}(\delta)\right]^{2}+\rho_{i} g^{\prime \prime}(\delta)\right\} d \delta_{t}^{2}
$$

and hence

$$
\frac{d C_{i t}}{C_{i t}}=\rho_{i} g^{\prime}(\delta) \mu d t+\frac{1}{2} e^{-\rho_{i} \log \left(\xi_{i}\right)+\rho_{i} g\left(\delta_{t}\right)}\left\{\left[\rho_{i} g^{\prime}(\delta)\right]^{2}+\rho_{i} g^{\prime \prime}(\delta)\right\} \sigma^{2} d t+\rho_{i} g^{\prime}(\delta) d Z_{t}
$$

or

$$
\frac{d C_{i t}}{C_{i t}}=\frac{\rho_{i} \mu}{E^{*}\left[\rho \mid \delta_{t}\right]}+\frac{1}{2}\left\{\left[\frac{\rho_{i}}{E^{*}\left[\rho \mid \delta_{t}\right]}\right]^{2}+\frac{\rho_{i}}{E^{*}\left[\rho \mid \delta_{t}\right]}\left(1-\frac{E^{* *}\left[\rho \mid \delta_{t}\right]}{E^{*}\left[\rho \mid \delta_{t}\right]}\right)\right\} \sigma^{2} d t+\frac{\rho_{i}}{E^{*}\left[\rho \mid \delta_{t}\right]} d Z_{t}
$$

or

$$
\frac{d C_{i t}}{C_{i t}}=\frac{\rho}{E^{*}\left[\rho \mid \delta_{t}\right]}\left\{\mu+\frac{1}{2}\left\{1+\frac{\rho_{i}-E^{* *}\left[\rho \mid \delta_{t}\right]}{E^{*}\left[\rho \mid \delta_{t}\right]}\right\} \sigma^{2}\right\} d t+\frac{\rho_{i}}{E^{*}\left[\rho \mid \delta_{t}\right]} d Z_{t}
$$

Q.E.D.

Proof of Proposition 3. The stochastic discount factor is

$$
M_{t}=e^{-\phi t-g\left(\delta_{t}\right)}
$$

Therefore

$$
d M_{t}=-\phi M d t-g^{\prime}(\delta) M_{t} d \delta+\frac{1}{2}\left[g^{\prime}\left(\delta_{t}\right)^{2}-g^{\prime \prime}\left(\delta_{t}\right)\right] M_{t} d \delta^{2}
$$

Hence

$$
\frac{d M_{t}}{M_{t}}=-\phi d t-g^{\prime}(\delta) \mu d t+\frac{1}{2}\left[g^{\prime}\left(\delta_{t}\right)^{2}-g^{\prime \prime}\left(\delta_{t}\right)\right] \sigma^{2} d t-g^{\prime}(\delta) \sigma d Z_{t}
$$

or

$$
\frac{d M_{t}}{M_{t}}=-\left\{\phi+g^{\prime}(\delta) \mu-\frac{1}{2}\left[g^{\prime}\left(\delta_{t}\right)^{2}-g^{\prime \prime}\left(\delta_{t}\right)\right] \sigma^{2}\right\} d t-g^{\prime}(\delta) \sigma d Z_{t}
$$

obtaining

$$
\frac{d M_{t}}{M_{t}}=-r\left(\delta_{t}\right) d t-\sigma_{M}\left(\delta_{t}\right) d Z_{t}
$$

with

$$
\begin{aligned}
r\left(\delta_{t}\right) & =\phi+g^{\prime}(\delta) \mu-\frac{1}{2}\left[g^{\prime}\left(\delta_{t}\right)^{2}-g^{\prime \prime}\left(\delta_{t}\right)\right] \sigma^{2} \\
& =\phi+\frac{1}{E^{*}\left[\rho \mid \delta_{t}\right]}\left\{\mu-\frac{1}{2}\left[\frac{1+E^{* *}\left[\rho \mid \delta_{t}\right]}{E^{*}\left[\rho \mid \delta_{t}\right]}-1\right] \sigma^{2}\right\} \\
\sigma_{M}(\delta) & =\frac{\sigma}{E^{*}\left[\rho \mid \delta_{t}\right]}
\end{aligned}
$$

Q.E.D. 
Proof of Proposition 4. The pricing formula stems from the pricing equation

$$
\begin{aligned}
P_{t} & =E_{t}\left[\int_{t}^{T} \frac{M_{\tau}}{M_{t}} D_{\tau} d \tau\right]=e^{g\left(\delta_{t+\tau}\right)} E_{t}\left[\int_{0}^{T-t} e^{-\phi \tau-g\left(\delta_{t+\tau}\right)+\delta_{t+\tau}} d \tau\right] \\
& =p(T-t) e^{g\left(\delta_{t}\right)} E_{t}\left[e^{\delta_{t+\tau}-g\left(\delta_{t+\tau}\right)}\right]
\end{aligned}
$$

Recall that

$$
\delta_{t+\tau}=\delta_{t}+\mu \tau+\sigma \sqrt{\tau} x \text { for } x \sim N(0,1)
$$

and therefore, $\frac{\partial g\left(\delta_{t+\tau}\right)}{\partial \delta_{t}}=g^{\prime}\left(\delta_{t+\tau}\right)$. We thus obtain that the

$$
\log \left(P_{t}\right)=\log (p(T-t))+g\left(\delta_{t}\right)+\log \left(E_{t}\left[e^{\delta_{t+\tau}-g\left(\delta_{t+\tau}\right)}\right]\right)
$$

This implies

$$
d \log \left(P_{t}\right)=o(d t)+\left\{g^{\prime}\left(\delta_{t}\right)+\frac{E_{t}\left[\left(1-g^{\prime}\left(\delta_{t+\tau}\right)\right) e^{\delta_{t+\tau}-g\left(\delta_{t+\tau}\right)}\right]}{E_{t}\left[e^{\delta_{t+\tau}-g\left(\delta_{t+\tau}\right)}\right]}\right\} \sigma d Z_{t}
$$

The results of proposition 4 follow. Q.E.D.

Proof of Corollary 5. The $\mathrm{P} / \mathrm{D}$ ratio is

$$
\frac{P}{D}=p(T-t) e^{g\left(\delta_{t}\right)-\delta_{t}} E_{t}\left[e^{\delta_{t+\tau}-g\left(\delta_{t+\tau}\right)}\right]
$$

Therefore

$$
\log (P / D)=\log p(T-t)+g\left(\delta_{t}\right)-\delta_{t}+\log \left(E_{t}\left[e^{\delta_{t+\tau}-g\left(\delta_{t+\tau}\right)}\right]\right)
$$

It follows

$$
\begin{aligned}
\frac{\partial \log (P / D)}{\partial \delta_{t}} & =g^{\prime}\left(\delta_{t}\right)-1+\frac{E_{t}\left[\left(1-g^{\prime}\left(\delta_{t+\tau}\right)\right) e^{\delta_{t+\tau}-g\left(\delta_{t+\tau}\right)}\right]}{E_{t}\left[e^{\delta_{t+\tau}-g\left(\delta_{t+\tau}\right)}\right]} \\
& =g^{\prime}\left(\delta_{t}\right)-\frac{E_{t}\left[g^{\prime}\left(\delta_{t+\tau}\right) e^{\delta_{t+\tau}-g\left(\delta_{t+\tau}\right)}\right]}{E_{t}\left[e^{\delta_{t+\tau}-g\left(\delta_{t+\tau}\right)}\right]} \\
& =g^{\prime}\left(\delta_{t}\right)-\widetilde{E}_{t}^{x, \tau}\left[g^{\prime}\left(\delta_{t+\tau}\right)\right]>0
\end{aligned}
$$

if and only if

$$
g^{\prime}\left(\delta_{t}\right)>\widetilde{E}_{t}^{x, \tau}\left[g^{\prime}\left(\delta_{t+\tau}\right)\right]
$$

Establishing the first result.

As for the second result, note that

$$
g^{\prime}\left(\delta_{t}\right)-\frac{E_{t}\left[g^{\prime}\left(\delta_{t+\tau}\right) e^{\delta_{t+\tau}-g\left(\delta_{t+\tau}\right)}\right]}{E_{t}\left[e^{\delta_{t+\tau}-g\left(\delta_{t+\tau}\right)}\right]}>0
$$


iff

$$
\frac{g^{\prime}\left(\delta_{t}\right) E_{t}\left[e^{\delta_{t+\tau}-g\left(\delta_{t+\tau}\right)}\right]-E_{t}\left[g^{\prime}\left(\delta_{t+\tau}\right) e^{\delta_{t+\tau}-g\left(\delta_{t+\tau}\right)}\right]}{E_{t}\left[e^{\delta_{t+\tau}-g\left(\delta_{t+\tau}\right)}\right]}>0
$$

iff

$$
\frac{E_{t}\left[\left[g^{\prime}\left(\delta_{t}\right)-g^{\prime}\left(\delta_{t+\tau}\right)\right] e^{\delta_{t+\tau}-g\left(\delta_{t+\tau}\right)}\right]}{E_{t}\left[e^{\delta_{t+\tau}-g\left(\delta_{t+\tau}\right)}\right]}=\widetilde{E}_{t}^{x, \tau}\left[g^{\prime}\left(\delta_{t}\right)-g^{\prime}\left(\delta_{t+\tau}\right)\right]>0
$$

Recall that $g^{\prime}\left(\delta_{t}\right)$ is decreasing, therefore $\delta_{t+\tau}>\delta_{t}$ implies $\left[g^{\prime}\left(\delta_{t}\right)-g^{\prime}\left(\delta_{t+\tau}\right)\right]>0$. The distribution of $\delta_{t+\tau}$ is

$$
f\left(\delta_{t+\tau}\right)=\phi\left(\delta_{t+\tau} ; \delta_{t}+\mu \tau ; \sigma^{2} \tau \mid \tau\right) f_{\tau}(\tau)
$$

and the distribution

$$
\widetilde{f}\left(\delta_{t+\tau}\right)=\frac{\phi\left(\delta_{t+\tau} ; \delta_{t}+\mu \tau ; \sigma^{2} \tau \mid \tau\right) e^{\delta_{t+\tau}-g\left(\delta_{t+\tau}\right)}}{E\left[e^{\delta_{t+\tau}-g\left(\delta_{t+\tau}\right)}\right]} f_{\tau}(\tau)
$$

Note that $h\left(\delta_{t+\tau}\right)=\delta_{t+\tau}-g\left(\delta_{t+\tau}\right)$ has $h^{\prime}\left(\delta_{t+\tau}\right)=1-g^{\prime}\left(\delta_{t+\tau}\right)<0$ if $\rho_{H}<1$ and $h^{\prime}\left(\delta_{t+\tau}\right)=$ $1-g^{\prime}\left(\delta_{t+\tau}\right)>1$ if $\rho_{H}>1$ and $\delta_{t+\tau}$ is sufficiently high (above some threshold). If $\rho_{H}>1$ and $\delta_{t}$ is sufficiently high, then $\tilde{f}\left(\delta_{t+\tau}\right)$ gives more weight to high $\delta_{t+\tau}$ than $f\left(\delta_{t+\tau}\right)$. Given that the latter is symmetric around $\delta_{t}+\mu \tau$, it follows that for any $\mu>0$, the distribution $\tilde{f}\left(\delta_{t+\tau}\right)$ gives an increasingly higher weight to higher $\delta_{t+\tau}$ compared to the normal density. Thus, as the weight on $\delta_{t+\tau}$ increases compared to $\delta_{t}$ and eventually $\widetilde{E}_{t}^{x, \tau}\left[g^{\prime}\left(\delta_{t}\right)-g^{\prime}\left(\delta_{t+\tau}\right)\right]>0$.

When $h^{\prime}\left(\delta_{t+\tau}\right)=1-g^{\prime}\left(\delta_{t+\tau}\right)<0$, instead, then $\tilde{f}\left(\delta_{t+\tau}\right)$ gives more weights to lower $\delta_{t+\tau}$ than $f\left(\delta_{t+\tau}\right)$. However, so long $\mu / \sigma$ is sufficiently high and the distribution $\tau$ gives sufficiently high weights to high $\tau$, the conditional distribution $\phi\left(\delta_{t+\tau} ; \delta_{t}+\mu \tau ; \sigma^{2} \tau \mid \tau\right)$ gives low or even negligible weight to $\delta_{t+\tau}<\delta_{t}$. Indeed, the result is immediate if $\sigma \rightarrow 0$ and $\mu>0$ in which case $\phi\left(\delta_{t+\tau} ; \delta_{t}+\mu \tau ; \sigma^{2} \tau \mid \tau\right)$ gives zero weight to $\delta_{t+\tau}<\delta_{t}$ for every $\tau>0$. By continuity, it follows that if $\mu / \sigma$ is sufficiently high, $\widetilde{E}_{t}^{x, \tau}\left[g^{\prime}\left(\delta_{t}\right)-g^{\prime}\left(\delta_{t+\tau}\right)\right]>0$.

Q.E.D.

Proof of Proposition 5: The wealth of agent $i$ is

$$
\begin{aligned}
W_{i t} & =E_{t}\left[\int_{t}^{T} \frac{M_{\tau}}{M_{t}} C_{i \tau} d \tau\right]=e^{g\left(\delta_{t}\right)} E_{t}\left[\int_{t}^{T} e^{-\phi(\tau-t)-g\left(\delta_{\tau}\right)} e^{-\rho_{i} \log \left(\xi_{i}\right)+\rho_{i} g\left(\delta_{\tau}\right)} d \tau\right] \\
& =e^{-\rho_{i} \log \left(\xi_{i}\right)+g\left(\delta_{t}\right)} E_{t}\left[\int_{0}^{T-t} e^{-\phi \tau+\left(\rho_{i}-1\right) g\left(\delta_{t+\tau}\right)} d \tau\right] \\
& =p(T-t) e^{-\rho_{i} \log \left(\xi_{i}\right)+g\left(\delta_{t}\right)} E^{x, \tau}\left[e^{\left(\rho_{i}-1\right) g\left(\delta_{t+\tau}\right)}\right]
\end{aligned}
$$

The $\mathrm{C} / \mathrm{W}$ is thus

$$
\frac{C_{i t}}{W_{i t}}=\frac{e^{-\rho_{i} \log \left(\xi_{i}\right)+\rho_{i} g\left(\delta_{t}\right)}}{p(T-t) e^{-\rho_{i} \log \left(\xi_{i}\right)+g\left(\delta_{t}\right)} E^{x, \tau}\left[e^{\left(\rho_{i}-1\right) g\left(\delta_{t+\tau}\right)}\right]}
$$




$$
=\frac{1}{p(T-t) E^{x, \tau}\left[e^{\left(\rho_{i}-1\right)\left(g\left(\delta_{t+\tau}\right)-g\left(\delta_{t}\right)\right)}\right]}
$$

Q.E.D.

Proof of Corollary 8. Note that

$$
P_{t}=p(T-t) e^{+g\left(\delta_{t}\right)} E^{C S}\left[\lambda\left(\rho_{i}\right) E_{t}^{x, \tau}\left[e^{\left(\rho_{i}-1\right) g\left(\delta_{t+\tau}\right)}\right]\right]
$$

Therefore

$$
\omega_{i t}^{W}=\frac{W_{i t}}{P_{t}}=\frac{s_{i} \lambda\left(\rho_{i}\right) E_{t}^{x, \tau}\left[e^{\left(\rho_{i}-1\right) g\left(\delta_{t+\tau}\right)}\right]}{E^{C S}\left[s_{j} \lambda\left(\rho_{j}\right) E_{t}^{x, \tau}\left[e^{\left(\rho_{j}-1\right) g\left(\delta_{t+\tau}\right)}\right]\right]}
$$

Part (b) follows immediately from the definition of $\lambda\left(\rho_{i}\right)$ in $(9)$, which in the notation here is given by

$$
\lambda\left(\rho_{i}\right)=\frac{E^{C S}\left[s_{j} \lambda\left(\rho_{j}\right) E_{0}^{x, \tau}\left[e^{\left(\rho_{j}-1\right) g\left(\delta_{t+\tau}\right)}\right]\right]}{E_{0}^{x, \tau}\left[e^{\left(\rho_{i}-1\right) g\left(\delta_{t+\tau}\right)}\right]}
$$

Part (c) of Corollary 8 can be shown as follows

$$
\omega_{i t}=\frac{W_{i t}}{P_{t}}=\frac{e^{-\rho_{i} \log \left(\xi_{i}\right)} E^{x, \tau}\left[e^{\left(\rho_{i}-1\right) g\left(\delta_{t+\tau}\right)}\right]}{E^{x, \tau}\left[e^{\delta_{t+\tau}-g\left(\delta_{t+\tau}\right)}\right]}
$$

Therefore

$$
\log \left(\omega_{i t}\right)=-\rho_{i} \log \left(\xi_{i}\right)+\log \left(E^{x, \tau}\left[e^{\left(\rho_{i}-1\right) g\left(\delta_{t+\tau}\right)}\right]\right)-\log \left(E^{x, \tau}\left[e^{\delta_{t+\tau}-g\left(\delta_{t+\tau}\right)}\right]\right)
$$

Thus

$$
\begin{aligned}
\frac{\partial \log \left(\omega_{i t}^{W}\right)}{\partial \delta_{t}} & =\frac{E^{x, \tau}\left[\left(\rho_{i}-1\right) g^{\prime}\left(\delta_{t+\tau}\right) e^{\left(\rho_{i}-1\right) g\left(\delta_{t+\tau}\right)}\right]}{E^{x, \tau}\left[e^{\left(\rho_{i}-1\right) g\left(\delta_{t+\tau}\right)}\right]}-\frac{E^{x, \tau}\left[\left(1-g^{\prime}\left(\delta_{t+\tau}\right)\right) e^{\delta_{t+\tau}-g\left(\delta_{t+\tau}\right)}\right]}{E^{x, \tau}\left[e^{\delta_{t+\tau}-g\left(\delta_{t+\tau}\right)}\right]} \\
& =\left(\rho_{i}-1\right) \frac{E^{x, \tau}\left[g^{\prime}\left(\delta_{t+\tau}\right) e^{\left(\rho_{i}-1\right) g\left(\delta_{t+\tau}\right)}\right]}{E^{x, \tau}\left[e^{\left(\rho_{i}-1\right) g\left(\delta_{t+\tau}\right)}\right]}-1+\frac{E^{x, \tau}\left[g^{\prime}\left(\delta_{t+\tau}\right) e^{\delta_{t+\tau}-g\left(\delta_{t+\tau}\right)}\right]}{E^{x, \tau}\left[e^{\delta_{t+\tau}-g\left(\delta_{t+\tau}\right)}\right]}>0
\end{aligned}
$$

iff

$$
\operatorname{LHS}(\rho)=\left(\rho_{i}-1\right) \frac{E^{x, \tau}\left[g^{\prime}\left(\delta_{t+\tau}\right) e^{\left(\rho_{i}-1\right) g\left(\delta_{t+\tau}\right)}\right]}{E^{x, \tau}\left[e^{\left(\rho_{i}-1\right) g\left(\delta_{t+\tau}\right)}\right]}>1-\frac{E^{x, \tau}\left[g^{\prime}\left(\delta_{t+\tau}\right) e^{\delta_{t+\tau}-g\left(\delta_{t+\tau}\right)}\right]}{E^{x, \tau}\left[e^{\delta_{t+\tau}-g\left(\delta_{t+\tau}\right)}\right]}
$$

The RHS does not depend on $\rho_{i}$. The LHS increases in $\rho$ for $\rho<\rho^{*}$ with $\rho^{*}>1$. In fact,

$$
\begin{aligned}
& \frac{\partial \operatorname{LHS}(\rho)}{\partial \rho}= \\
= & \frac{E^{x, \tau}\left[g^{\prime}\left(\delta_{t+\tau}\right) e^{\left(\rho_{i}-1\right) g\left(\delta_{t+\tau}\right)}\right]}{E^{x, \tau}\left[e^{\left(\rho_{i}-1\right) g\left(\delta_{t+\tau}\right)}\right]} \\
& +\left(\rho_{i}-1\right)\left\{\frac{E^{x, \tau}\left[g^{\prime}\left(\delta_{t+\tau}\right) g\left(\delta_{t+\tau}\right) e^{\left(\rho_{i}-1\right) g\left(\delta_{t+\tau}\right)}\right]}{E^{x, \tau}\left[e^{\left(\rho_{i}-1\right) g\left(\delta_{t+\tau}\right)}\right]}-\frac{E^{x, \tau}\left[g^{\prime}\left(\delta_{t+\tau}\right) e^{\left(\rho_{i}-1\right) g\left(\delta_{t+\tau}\right)}\right] E^{x, \tau}\left[g\left(\delta_{t+\tau}\right) e^{\left(\rho_{i}-1\right) g\left(\delta_{t+\tau}\right)}\right]}{E^{x, \tau}\left[e^{\left(\rho_{i}-1\right) g\left(\delta_{t+\tau}\right)}\right]^{2}}\right\} \\
> & 0
\end{aligned}
$$


iff

$$
\begin{aligned}
\frac{\partial L H S(\rho)}{\partial \rho}= & \frac{E^{x, \tau}\left[g^{\prime}\left(\delta_{t+\tau}\right) e^{\left(\rho_{i}-1\right) g\left(\delta_{t+\tau}\right)}\right]}{E^{x, \tau}\left[e^{\left(\rho_{i}-1\right) g\left(\delta_{t+\tau}\right)}\right]} \\
& +\left(\rho_{i}-1\right)\left\{\widetilde{E}_{i}^{x, \tau}\left[g^{\prime}\left(\delta_{t+\tau}\right) g\left(\delta_{t+\tau}\right)\right]-\widetilde{E}_{i}^{x, \tau}\left[g^{\prime}\left(\delta_{t+\tau}\right)\right] \widetilde{E}_{i}^{x, \tau}\left[g\left(\delta_{t+\tau}\right)\right]\right\} \\
> & 0
\end{aligned}
$$

We thus have that

$$
\begin{aligned}
\frac{\partial \operatorname{LHS}(\rho)}{\partial \rho} & =\frac{E^{x, \tau}\left[g^{\prime}\left(\delta_{t+\tau}\right) e^{\left(\rho_{i}-1\right) g\left(\delta_{t+\tau}\right)}\right]}{E^{x, \tau}\left[e^{\left(\rho_{i}-1\right) g\left(\delta_{t+\tau}\right)}\right]}+\left(\rho_{i}-1\right)\left\{\widetilde{\operatorname{Cov}}_{i}^{x, \tau}\left[g^{\prime}\left(\delta_{t+\tau}\right) g\left(\delta_{t+\tau}\right)\right]\right\} \\
& =(+)+(-)(-) \\
& >0
\end{aligned}
$$

is always true for $\rho_{i}<1$, as $g^{\prime}\left(\delta_{t+\tau}\right)$ is decreasing while $g\left(\delta_{t+\tau}\right)$ is increasing. Because $\frac{\partial L H S(\rho)}{\partial \rho}>0$ (strictly) for $\rho_{i}=1$, by continuity this also holds for all $\rho_{i}<\rho^{*}$ for some threshold $\rho^{*}>1$.

Two additional points are useful

1. Note now that for $\rho=1$ (log utility), the $L H S=0$. Therefore, the condition for $\log$ utility investor is $0>1-\widetilde{E}_{t}^{x, \tau}\left[g^{\prime}\left(\delta_{t+\tau}\right)\right]$. Below in Lemma A1 I prove this threshold is increasing (ie. $H(\delta)=\widetilde{E}_{t}^{x, \tau}\left[g^{\prime}\left(\delta_{t+\tau}\right)\right]$ decreasing) and therefore the wealth share of log-utility investor is initially increasing in $\delta$ but at some point it starts to decrease. That is, the log utility investor initially is moving up but eventually he moves down the wealth distribution. By continuity, the same argument applies to a whole set of agents around log-utility.

2. Note that as $\delta_{t}$ increases, both expectations in

$$
\begin{aligned}
\operatorname{LHS}(\rho) & =\left(\rho_{i}-1\right) \frac{E^{x, \tau}\left[g^{\prime}\left(\delta_{t+\tau}\right) e^{\left(\rho_{i}-1\right) g\left(\delta_{t+\tau}\right)}\right]}{E^{x, \tau}\left[e^{\left(\rho_{i}-1\right) g\left(\delta_{t+\tau}\right)}\right]}>1-\frac{E^{x, \tau}\left[g^{\prime}\left(\delta_{t+\tau}\right) e^{\delta_{t+\tau}-g\left(\delta_{t+\tau}\right)}\right]}{E^{x, \tau}\left[e^{\delta_{t+\tau}-g\left(\delta_{t+\tau}\right)}\right]} \\
& =\left(\rho_{i}-1\right) \widetilde{E}_{i}^{x, \tau}\left[g^{\prime}\left(\delta_{t+\tau}\right)\right]>1-\widetilde{E}^{x, \tau}\left[g^{\prime}\left(\delta_{t+\tau}\right)\right]
\end{aligned}
$$

converge to $1 / \rho_{H}$, obtaining the condition

$$
\rho_{i}>\rho_{H}
$$

which is never satisfied. So, eventually, for all $i$ with $\rho_{i}<\rho_{H}$, we must have $\frac{\partial \log \left(\omega_{i t}^{W}\right)}{\partial \delta_{t}}<$ 0 . All agents eventually have a wealth share that is declining in output. 
Proof of Part (d). Consider

$$
E^{C S}\left[\omega_{i t}^{W} \mid \rho_{i}>\underline{\rho}\right]
$$

We have

$$
\int_{i: \rho_{i}>\underline{\rho}} \frac{W_{i t}}{P} d i=m\left(\rho_{i}>\underline{\rho}\right) E^{C S}\left[\omega_{i t}^{W} \mid \rho_{i}>\underline{\rho}\right]
$$

where $m\left(\rho_{i}>\underline{\rho}\right)$ is the mass of agents with risk tolerance higher than $\underline{\rho}$. We have

$$
E^{C S}\left[\omega_{i t}^{W} \mid \rho_{i}>\underline{\rho}\right]=\frac{E^{C S}\left[s_{i} \lambda\left(\rho_{i}\right) E^{x, \tau}\left[e^{\left(\rho_{i}-1\right) g\left(\delta_{t+\tau}\right)}\right] \mid \rho_{i}>\underline{\rho}\right]}{E^{C S}\left[s_{j} \lambda\left(\rho_{j}\right) E^{x, \tau}\left[e^{\left(\rho_{j}-1\right) g\left(\delta_{t+\tau}\right)}\right]\right]}
$$

and thus

$$
\begin{aligned}
& E^{C S}\left[\omega_{i t}^{W} \mid \rho_{i}>\underline{\rho}\right] \\
= & \frac{E^{C S}\left[s_{i} \lambda\left(\rho_{i}\right) E^{x, \tau}\left[e^{\left(\rho_{i}-1\right) g\left(\delta_{t+\tau}\right)}\right] \mid \rho_{i}>\underline{\rho}\right]}{E^{C S}\left[s_{j} \lambda\left(\rho_{j}\right) E^{x, \tau}\left[e^{\left(\rho_{j}-1\right) g\left(\delta_{t+\tau}\right)}\right]\right]} \\
= & \frac{E^{C S}\left[s_{i} \lambda\left(\rho_{i}\right) E^{x, \tau}\left[e^{\left(\rho_{i}-1\right) g\left(\delta_{t+\tau}\right)}\right] \mid \rho_{i}>\underline{\rho}\right]}{E^{C S}\left[s_{j} \lambda\left(\rho_{j}\right) E^{x, \tau}\left[e^{\left(\rho_{j}-1\right) g\left(\delta_{t+\tau}\right)}\right] \mid \rho_{j}>\underline{\rho}\right] m\left(\rho_{j}>\underline{\rho}\right)+E^{C S}\left[s_{j} \lambda\left(\rho_{j}\right) E^{x, \tau}\left[e^{\left(\rho_{j}-1\right) g\left(\delta_{t+\tau}\right)}\right] \mid \rho_{j}<\underline{\rho}\right]\left[1-m\left(\rho_{j}>\underline{\rho}\right)\right]} \\
= & \frac{1}{m\left(\rho_{j}>\underline{\rho}\right)+\frac{E^{C S}\left[s_{j} \lambda\left(\rho_{j}\right) E^{x, \tau}\left[e^{\left(\rho_{j}-1\right) g\left(\delta_{t+\tau}\right)}\right] \mid \rho_{j}<\underline{\rho}\right]}{E^{C S}\left[s_{i} \lambda\left(\rho_{i}\right) E^{x, \tau}\left[e^{\left(\rho_{i}-1\right) g\left(\delta_{t+\tau}\right)}\right] \mid \rho_{i}>\underline{\rho}\right]}\left[1-m\left(\rho_{j}>\underline{\rho}\right)\right]}
\end{aligned}
$$

Consider now the ratio

$$
\begin{aligned}
R\left(\delta_{t}\right) & =\frac{E^{C S}\left[s_{j} \lambda\left(\rho_{j}\right) E_{t}^{x, \tau}\left[e^{\left(\rho_{j}-1\right) g\left(\delta_{t+\tau}\right)}\right] \mid \rho_{j}<\underline{\rho}\right]}{E^{C S}\left[s_{i} \lambda\left(\rho_{i}\right) E_{t}^{x, \tau}\left[e^{\left(\rho_{i}-1\right) g\left(\delta_{t+\tau}\right)}\right] \mid \rho_{i}>\underline{\rho}\right]} \\
& =\frac{E^{C S}\left[s_{j} \lambda\left(\rho_{j}\right) \mid \rho_{j}<\underline{\rho}\right]}{E^{C S}\left[s_{i} \lambda\left(\rho_{i}\right) \mid \rho_{i}>\underline{\rho}\right]} \frac{\frac{E^{C S}\left[s_{j} \lambda\left(\rho_{j}\right) E_{t}^{x, \tau}\left[e^{\left(\rho_{j}-1\right) g\left(\delta_{t+\tau}\right)}\right] \mid \rho_{j}<\underline{\rho}\right]}{E^{C S}\left[s_{j} \lambda\left(\rho_{j}\right) \mid \rho_{j}<\underline{\rho}\right]}}{\frac{E^{C S}\left[s_{i} \lambda\left(\rho_{i}\right) E_{t}^{x, \tau}\left[e^{\left(\rho_{i}-1\right) g\left(\delta_{t+\tau}\right)}\right] \mid \rho_{i}>\underline{\rho}\right]}{E^{C S}\left[s_{i} \lambda\left(\rho_{i}\right) \mid \rho_{i}>\underline{\rho}\right]}}
\end{aligned}
$$

so that we can write

$$
R\left(\delta_{t}\right)=\left(\frac{E^{C S}\left[s_{j} \lambda\left(\rho_{j}\right) \mid \rho_{j}<\underline{\rho}\right]}{E^{C S}\left[s_{i} \lambda\left(\rho_{i}\right) \mid \rho_{i}>\underline{\rho}\right]}\right)\left(\frac{\widehat{E}^{C S}\left[E_{t}^{x, \tau}\left[e^{\left(\rho_{j}-1\right) g\left(\delta_{t+\tau}\right)}\right] \mid \rho_{j}<\underline{\rho}\right]}{\widehat{E}^{C S}\left[E_{t}^{x, \tau}\left[e^{\left(\rho_{i}-1\right) g\left(\delta_{t+\tau}\right)}\right] \mid \rho_{i}>\underline{\rho}\right]}\right)
$$

where

$$
\begin{aligned}
\widehat{E}^{C S}\left[E_{t}^{x, \tau}\left[e^{\left(\rho_{j}-1\right) g\left(\delta_{t+\tau}\right)}\right] \mid \rho_{j}<\underline{\rho}\right] & =\frac{E^{C S}\left[s_{j} \lambda\left(\rho_{j}\right) E_{t}^{x, \tau}\left[e^{\left(\rho_{j}-1\right) g\left(\delta_{t+\tau}\right)}\right] \mid \rho_{j}<\underline{\rho}\right]}{E^{C S}\left[s_{j} \lambda\left(\rho_{j}\right) \mid \rho_{j}<\underline{\rho}\right]} \\
\widehat{E}^{C S}\left[E_{t}^{x, \tau}\left[e^{\left(\rho_{i}-1\right) g\left(\delta_{t+\tau}\right)}\right] \mid \rho_{i}>\underline{\rho}\right] & =\frac{E^{C S}\left[s_{i} \lambda\left(\rho_{i}\right) E_{t}^{x, \tau}\left[e^{\left(\rho_{i}-1\right) g\left(\delta_{t+\tau}\right)}\right] \mid \rho_{i}>\underline{\rho}\right]}{E^{C S}\left[s_{i} \lambda\left(\rho_{i}\right) \mid \rho_{i}>\underline{\rho}\right]}
\end{aligned}
$$

are just normalization so that we can deal with simple cross-sectional averages over $\rho_{i}$ conditional on the latter being above or below a threshold. 
The first term in $R\left(\delta_{t}\right)$ does not depend on $\delta_{t}$. Both the numerator and denominator of the second term depend on $g\left(\delta_{t}\right)$. Because $g\left(\delta_{t}\right)$ is increasing and hence $g\left(\delta_{t+\tau}\right)>0$ for $\delta_{t}$ sufficiently high, an increase in $g\left(\delta_{t+\tau}\right)$ induces an increase in the denominator that is higher than the impact at the numerator, because $\rho_{j}$ are uniformly higher at the denominator. Thus, $R\left(\delta_{t}\right)$ is decreasing in $\delta_{t}$ and hence $E\left[\omega^{W} \mid \rho>\underline{\rho}\right]$ is increasing in $\delta$. The share of wealth to low risk-tolerance agents increase over time. Formally (and leaving out the first positive constant for notational simplicity)

$$
\begin{aligned}
& R^{\prime}\left(\delta_{t}\right) \\
= & \frac{\widehat{E}^{C S}\left[E_{t}^{x, \tau}\left[\left(\rho_{j}-1\right) g^{\prime}\left(\delta_{t+\tau}\right) e^{\left(\rho_{j}-1\right) g\left(\delta_{t+\tau}\right)}\right] \mid \rho_{j}<\underline{\rho}\right]}{\left(\widehat{E}^{C S}\left[E_{t}^{x, \tau}\left[e^{\left(\rho_{i}-1\right) g\left(\delta_{t+\tau}\right)}\right] \mid \rho_{i}>\underline{\rho}\right]\right)} \\
& -\frac{\widehat{E}^{C S}\left[E_{t}^{x, \tau}\left[e^{\left(\rho_{j}-1\right) g\left(\delta_{t+\tau}\right)}\right] \mid \rho_{j}<\underline{\rho}\right] \widehat{E}^{C S}\left[E_{t}^{x, \tau}\left[\left(\rho_{i}-1\right) g^{\prime}\left(\delta_{t+\tau}\right) e^{\left(\rho_{i}-1\right) g\left(\delta_{t+\tau}\right)}\right] \mid \rho_{i}>\underline{\rho}\right]}{\left(\widehat{E}^{C S}\left[E_{t}^{x, \tau}\left[e^{\left(\rho_{i}-1\right) g\left(\delta_{t+\tau}\right)}\right] \mid \rho_{i}>\underline{\rho}\right]\right)^{2}} \\
= & \frac{\widehat{E}^{C S}\left[E_{t}^{x, \tau}\left[e^{\left(\rho_{j}-1\right) g\left(\delta_{t+\tau}\right)}\right] \mid \rho_{j}<\underline{\rho}\right]}{\widehat{E}^{C S}\left[E_{t}^{x, \tau}\left[e^{\left(\rho_{i}-1\right) g\left(\delta_{t+\tau}\right)}\right] \mid \rho_{i}>\underline{\rho}\right]} \times \\
& \times\left(\frac{\widehat{E}^{C S}\left[E_{t}^{x, \tau}\left[\left(\rho_{j}-1\right) g^{\prime}\left(\delta_{t+\tau}\right) e^{\left(\rho_{j}-1\right) g\left(\delta_{t+\tau}\right)}\right] \mid \rho_{j}<\underline{\rho}\right]}{\widehat{E}^{C S}\left[E_{t}^{x, \tau}\left[e^{\left(\rho_{j}-1\right) g\left(\delta_{t+\tau}\right)}\right] \mid \rho_{j}<\underline{\rho}\right]}-\frac{\widehat{E}^{C S}\left[E_{t}^{x, \tau}\left[\left(\rho_{i}-1\right) g^{\prime}\left(\delta_{t+\tau}\right) e^{\left(\rho_{i}-1\right) g\left(\delta_{t+\tau}\right)}\right] \mid \rho_{i}>\underline{\rho}\right]}{\widehat{E}^{C S}\left[E_{t}^{x, \tau}\left[e^{\left(\rho_{i}-1\right) g\left(\delta_{t+\tau}\right)}\right] \mid \rho_{i}>\underline{\rho}\right]}\right) \\
= & \frac{\widehat{E}^{C S}\left[E_{t}^{x, \tau}\left[e^{\left(\rho_{j}-1\right) g\left(\delta_{t+\tau}\right)}\right] \mid \rho_{j}<\underline{\rho}\right]}{\widehat{E}^{C S}\left[E_{t}^{x, \tau}\left[e^{\left(\rho_{i}-1\right) g\left(\delta_{t+\tau}\right)}\right] \mid \rho_{i}>\underline{\rho}\right]}\left(\widehat{\widehat{E}}^{C S}\left[E_{t}^{x, \tau}\left[\left(\rho_{j}-1\right) g^{\prime}\left(\delta_{t+\tau}\right)\right] \mid \rho_{j}<\underline{\rho}\right]-\widehat{\widehat{E}}^{C S}\left[E_{t}^{x, \tau}\left[\left(\rho_{i}-1\right) g^{\prime}\left(\delta_{t+\tau}\right)\right] \mid \rho_{i}>\underline{\rho}\right]\right) \\
< & 0
\end{aligned}
$$

where $\widehat{\widehat{E}}^{C S}\left[\right.$. ] expression use the relevant density weighted by $e^{\left(\rho_{j}-1\right) g\left(\delta_{t+\tau}\right)}$. The inequality stems from the fact that $g^{\prime}(\delta)>0$ and that the first term takes an average with $\rho_{j}$ that are uniformly lower than the $\rho_{i}$ in the second term. Thus, the first term must be lower than the second term, and the inequality follows.

Q.E.D.

\section{Lemma A1.}

$$
H\left(\delta_{t}\right)=\widetilde{E}_{t}^{x, \tau}\left[g^{\prime}\left(\delta_{t+\tau}\right)\right]=\frac{E^{x, \tau}\left[g^{\prime}\left(\delta_{t+\tau}\right) e^{\delta_{t+\tau}-g\left(\delta_{t+\tau}\right)}\right]}{E^{x, \tau}\left[e^{\delta_{t+\tau}-g\left(\delta_{t+\tau}\right)}\right]}
$$

is decreasing in $\delta$.

Proof. We have

$$
\begin{aligned}
H^{\prime}(\delta)= & \frac{E^{x, \tau}\left[g^{\prime \prime}\left(\delta_{t+\tau}\right) e^{\delta_{t+\tau}-g\left(\delta_{t+\tau}\right)}\right]}{E^{x, \tau}\left[e^{\delta_{t+\tau}-g\left(\delta_{t+\tau}\right)}\right]} \\
& +\frac{E^{x, \tau}\left[g^{\prime}\left(\delta_{t+\tau}\right)\left(1-g^{\prime}\left(\delta_{t+\tau}\right)\right) e^{\delta_{t+\tau}-g\left(\delta_{t+\tau}\right)}\right]}{E^{x, \tau}\left[e^{\delta_{t+\tau}-g\left(\delta_{t+\tau}\right)}\right]}-\frac{E^{x, \tau}\left[g^{\prime}\left(\delta_{t+\tau}\right) e^{\delta_{t+\tau}-g\left(\delta_{t+\tau}\right)}\right] E^{x, \tau}\left[\left(1-g^{\prime}\left(\delta_{t+\tau}\right)\right) e^{\delta_{t+\tau}-g\left(\delta_{t+\tau}\right)}\right]}{\left(E^{x, \tau}\left[e^{\delta_{t+\tau}-g\left(\delta_{t+\tau}\right)}\right]\right)^{2}} \\
= & \widetilde{E}^{x, \tau}\left[g^{\prime \prime}\left(\delta_{t+\tau}\right)\right] \\
& +\frac{E^{x, \tau}\left[g^{\prime}\left(\delta_{t+\tau}\right) e^{\delta_{t+\tau}-g\left(\delta_{t+\tau}\right)}\right]-E^{x, \tau}\left[\left[g^{\prime}\left(\delta_{t+\tau}\right)\right]^{2} e^{\delta_{t+\tau}-g\left(\delta_{t+\tau}\right)}\right]}{E^{x, \tau}\left[e^{\delta_{t+\tau}-g\left(\delta_{t+\tau}\right)}\right]}
\end{aligned}
$$




$$
\begin{aligned}
& -\frac{E^{x, \tau}\left[g^{\prime}\left(\delta_{t+\tau}\right) e^{\delta_{t+\tau}-g\left(\delta_{t+\tau}\right)}\right] E^{x, \tau}\left[e^{\delta_{t+\tau}-g\left(\delta_{t+\tau}\right)}\right]-E^{x, \tau}\left[g^{\prime}\left(\delta_{t+\tau}\right) e^{\delta_{t+\tau}-g\left(\delta_{t+\tau}\right)}\right] E^{x, \tau}\left[g^{\prime}\left(\delta_{t+\tau}\right) e^{\delta_{t+\tau}-g\left(\delta_{t+\tau}\right)}\right]}{E^{x, \tau}\left[e^{\delta_{t+\tau}-g\left(\delta_{t+\tau}\right)}\right] E^{x, \tau}\left[e^{\delta_{t+\tau}-g\left(\delta_{t+\tau}\right)}\right]} \\
= & \widetilde{E}^{x, \tau}\left[g^{\prime \prime}\left(\delta_{t+\tau}\right)\right] \\
& +\frac{E^{x, \tau}\left[g^{\prime}\left(\delta_{t+\tau}\right) e^{\delta_{t+\tau}-g\left(\delta_{t+\tau}\right)}\right]}{E^{x, \tau}\left[e^{\delta_{t+\tau}-g\left(\delta_{t+\tau}\right)}\right]}-\frac{E^{x, \tau}\left[\left[g^{\prime}\left(\delta_{t+\tau}\right)\right]^{2} e^{\delta_{t+\tau}-g\left(\delta_{t+\tau}\right)}\right]}{E^{x, \tau}\left[e^{\delta_{t+\tau}-g\left(\delta_{t+\tau}\right)}\right]} \\
& -\frac{E^{x, \tau}\left[g^{\prime}\left(\delta_{t+\tau}\right) e^{\delta_{t+\tau}-g\left(\delta_{t+\tau}\right)}\right] E^{x, \tau}\left[e^{\delta_{t+\tau}-g\left(\delta_{t+\tau}\right)}\right]}{E^{x, \tau}\left[e^{\delta_{t+\tau}-g\left(\delta_{t+\tau}\right)}\right] E^{x, \tau}\left[e^{\delta_{t+\tau}-g\left(\delta_{t+\tau}\right)}\right]}+\left(\frac{E^{x, \tau}\left[g^{\prime}\left(\delta_{t+\tau}\right) e^{\delta_{t+\tau}-g\left(\delta_{t+\tau}\right)}\right]}{E^{x, \tau}\left[e^{\delta_{t+\tau}-g\left(\delta_{t+\tau}\right)}\right]}\right)^{2} \\
= & \widetilde{E}^{x, \tau}\left[g^{\prime \prime}\left(\delta_{t+\tau}\right)\right]-\left\{\frac{E^{x, \tau}\left[\left[g^{\prime}\left(\delta_{t+\tau}\right)\right]^{2} e^{\delta_{t+\tau}-g\left(\delta_{t+\tau}\right)}\right]}{E^{x, \tau}\left[e^{\delta_{t+\tau}-g\left(\delta_{t+\tau}\right)}\right]}-\left(\frac{E^{x, \tau}\left[g^{\prime}\left(\delta_{t+\tau}\right) e^{\delta_{t+\tau}-g\left(\delta_{t+\tau}\right)}\right]}{E^{x, \tau}\left[e^{\delta_{t+\tau}-g\left(\delta_{t+\tau}\right)}\right]}\right)^{2}\right\} \\
= & \widetilde{E}^{x, \tau}\left[g^{\prime \prime}\left(\delta_{t+\tau}\right)\right]-\left\{\widetilde{E}^{x, \tau}\left[\left[g^{\prime}\left(\delta_{t+\tau}\right)\right]^{2}\right]-\left(E^{x, \tau}\left[g^{\prime}\left(\delta_{t+\tau}\right)\right]\right)^{2}\right\} \\
= & \widetilde{E}^{x, \tau}\left[g^{\prime \prime}\left(\delta_{t+\tau}\right)\right]-\widetilde{V a r}_{x, \tau}\left[g^{\prime}\left(\delta_{t+\tau}\right)\right] \\
< & 0
\end{aligned}
$$

Q.E.D.

\section{Proof of Proposition 6:}

The wealth of agent $i$ is

$$
W_{i t}=p(T-t) s_{i} \lambda\left(\rho_{i}\right) e^{g\left(\delta_{t}\right)} E^{x, \tau}\left[e^{\left(\rho_{i}-1\right) g\left(\delta_{t+\tau}\right)}\right]
$$

Thus,

$$
\log \left(W_{i t}\right)=\log (p(T-t))+\log \left(s_{i}\right)+\log \left(\lambda\left(\rho_{i}\right)\right)+g\left(\delta_{t}\right)+\log \left(E^{x, \tau}\left[e^{\left(\rho_{i}-1\right) g\left(\delta_{t+\tau}\right)}\right]\right)
$$

By Ito's Lemman, the volatilty of $\log \left(W_{i t}\right)$

$$
\sigma_{W i t}=\left\{g^{\prime}(\delta)+\left(\rho_{i}-1\right) \frac{E^{x, \tau}\left[g^{\prime}\left(\delta_{t+\tau}\right) e^{\left(\rho_{i}-1\right) g\left(\delta_{t+\tau}\right)}\right]}{E^{x, \tau}\left[e^{\left(\rho_{i}-1\right) g\left(\delta_{t+\tau}\right)}\right]}\right\} \sigma
$$

The drift rate follows from no arbitrage as the fair compensation for a security that pays $C_{i t}$ over time

$$
E\left[\frac{d W_{i t}+C_{i t} d t}{W_{i t}}\right]=\left(r_{t}+\sigma_{W i t} \sigma_{M t}\right) d t
$$

Q.E.D.

Proof of Proposition 7: Consider the first derivative of $\sigma_{W i t}$ with respect to $\rho_{i}$

$$
\begin{aligned}
\frac{\partial \sigma_{W i t}}{\partial \rho_{i}}= & \sigma \frac{E^{x, \tau}\left[g^{\prime}\left(\delta_{t+\tau}\right) e^{\left(\rho_{i}-1\right) g\left(\delta_{t+\tau}\right)}\right]}{E^{x, \tau}\left[e^{\left(\rho_{i}-1\right) g\left(\delta_{t+\tau}\right)}\right]} \\
& +\sigma\left(\rho_{i}-1\right)\left\{\frac{E^{x, \tau}\left[g^{\prime}\left(\delta_{t+\tau}\right) g\left(\delta_{t+\tau}\right) e^{\left(\rho_{i}-1\right) g\left(\delta_{t+\tau}\right)}\right]}{E^{x, \tau}\left[e^{\left(\rho_{i}-1\right) g\left(\delta_{t+\tau}\right)}\right]}-\frac{E^{x, \tau}\left[g^{\prime}\left(\delta_{t+\tau}\right) e^{\left(\rho_{i}-1\right) g\left(\delta_{t+\tau}\right)}\right] E^{x, \tau}\left[g\left(\delta_{t+\tau}\right) e^{\left(\rho_{i}-1\right) g\left(\delta_{t+\tau}\right)}\right]}{\left(E^{x, \tau}\left[e^{\left(\rho_{i}-1\right) g\left(\delta_{t+\tau}\right)}\right]\right)^{2}}\right\}
\end{aligned}
$$


We can define the density

$$
\tilde{f}_{i}\left(\delta_{t+\tau}\right)=\frac{f\left(\delta_{t+\tau}\right) e^{\left(\rho_{i}-1\right) g\left(\delta_{t+\tau}\right)}}{E^{x, \tau}\left[e^{\left(\rho_{i}-1\right) g\left(\delta_{t+\tau}\right)}\right]}
$$

to obtain

$$
\begin{aligned}
\frac{\partial \sigma_{W i t}}{\partial \rho_{i}} & =\sigma \widetilde{E}_{i}^{x, \tau}\left[g^{\prime}\left(\delta_{t+\tau}\right)\right]+\sigma\left(\rho_{i}-1\right)\left\{\widetilde{E}_{i}^{x, \tau}\left[g^{\prime}\left(\delta_{t+\tau}\right) g\left(\delta_{t+\tau}\right)\right]-\widetilde{E}_{i}^{x, \tau}\left[g^{\prime}\left(\delta_{t+\tau}\right)\right] \widetilde{E}^{x, \tau}\left[g\left(\delta_{t+\tau}\right)\right]\right\} \\
& =\sigma\left\{\widetilde{E}_{i}^{x, \tau}\left[g^{\prime}\left(\delta_{t+\tau}\right)\right]+\left(\rho_{i}-1\right) \widetilde{\operatorname{Cov}_{i}^{x, \tau}}\left[g^{\prime}\left(\delta_{t+\tau}\right), g\left(\delta_{t+\tau}\right)\right]\right\}
\end{aligned}
$$

We have $g\left(\delta_{t+\tau}\right)$ is increasing and $g^{\prime}\left(\delta_{t+\tau}\right)$ decreasing. Therefore, $\widetilde{\operatorname{Cov}}_{i}^{x, \tau}\left[g^{\prime}\left(\delta_{t+\tau}\right), g\left(\delta_{t+\tau}\right)\right]<$ 0 . Because $g^{\prime}\left(\delta_{t+\tau}\right)>0$ we have

$$
\frac{\partial \sigma_{W i t}}{\partial \rho_{i}}>0 \text { if } \rho_{i} \leq 1
$$

In addition, for $\rho_{i}=1, \frac{\partial \sigma_{W i t}}{\partial \rho_{i}}>0$, strictly. It follows by continuity that there is a $\rho^{*}>1$ such that $\frac{\partial \sigma_{W i t}}{\partial \rho_{i}}>0$ for all $\rho_{i}<\rho^{*}$.

Part (b) of the proposition stems from continuity of $\sigma_{W i t}$ in $\rho_{i}$ and the fact that the average is always contained between extremes $\sigma_{P}=\int \omega_{i t} \sigma_{W i t} d i$. The threshold $\rho\left(\delta_{t}\right)$ depends on $\delta_{t}$ as both the weights $\omega_{i t}$ and the volatilities depend on it.

Q.E.D.

We also note that as $\delta_{t}$ increases, $g^{\prime}\left(\delta_{t+\tau}\right) \rightarrow \rho_{H}^{-1}$. Thus $\widetilde{\operatorname{Cov}_{i}^{x}}{ }^{x}\left[g^{\prime}\left(\delta_{t+\tau}\right), g\left(\delta_{t+\tau}\right)\right] \rightarrow 0$, while $\widetilde{E}_{i}^{x, \tau}\left[g^{\prime}\left(\delta_{t+\tau}\right)\right] \rightarrow \rho_{H}^{-1}>0$. It follows that for $\delta_{t}$ sufficiently large, $\frac{\partial \sigma_{W i t}}{\partial \rho_{i}}>0$ for all $\rho_{i}<\rho_{H}$.

Proof of Proposition 8: Immediate from Proposition 7 and the formula for beta. Q.E.D.

Proof of Proposition 9: Immediate from Proposition 7 and the formulas for portfolio allocation Q.E.D.

Proof of Proposition 10: The volatility of a log utility investor is

$$
\sigma_{W t}^{\rho=1}=g^{\prime}(\delta) \sigma
$$

Therefore

$$
\sigma_{W t}^{\rho=1}>\sigma_{P t}
$$


iff

$$
g^{\prime}(\delta) \sigma>\left\{1+g^{\prime}\left(\delta_{t}\right)-\frac{E_{t}^{x, \tau}\left[g^{\prime}\left(\delta_{t+\tau}\right) e^{\delta_{t+\tau}-g\left(\delta_{t+\tau}\right)}\right]}{E_{t}^{x, \tau}\left[e^{\delta_{t+\tau}-g\left(\delta_{t+\tau}\right)}\right]}\right\} \sigma
$$

or

$$
0>1-\frac{E_{t}^{x, \tau}\left[g^{\prime}\left(\delta_{t+\tau}\right) e^{\delta_{t+\tau}-g\left(\delta_{t+\tau}\right)}\right]}{E_{t}^{x, \tau}\left[e^{\delta_{t+\tau}-g\left(\delta_{t+\tau}\right)}\right]}
$$

or

$$
\frac{E_{t}^{x, \tau}\left[g^{\prime}\left(\delta_{t+\tau}\right) e^{\delta_{t+\tau}-g\left(\delta_{t+\tau}\right)}\right]}{E_{t}^{x, \tau}\left[e^{\delta_{t+\tau}-g\left(\delta_{t+\tau}\right)}\right]}>1
$$

or

$$
\widetilde{E}_{t}^{x, \tau}\left[g^{\prime}\left(\delta_{t+\tau}\right)\right]>1
$$

We have $g^{\prime}\left(\delta_{t+\tau}\right) \rightarrow 1 / \rho_{L}>1$ for $\delta_{t+\tau} \rightarrow-\infty$ and $g^{\prime}\left(\delta_{t+\tau}\right) \rightarrow 1 / \rho_{H}<1$ for $\delta_{t+\tau} \rightarrow+\infty$. Because from Lemma A1 the expectation $\widetilde{E}_{t}^{x, \tau}[$.$] is monotonically decreasing in \delta_{t}$, the result follows by continuity. Q.E.D.

Proof of Corollary 10. The equilibrium condition for $g\left(\delta_{t}\right)$ is that it satisfies

$$
\begin{aligned}
1 & =\int f_{k}(\rho) \lambda_{k}(\rho) e^{\rho g_{k}\left(\delta_{t}\right)-\delta_{t}} d \rho \\
\lambda_{k}(\rho) & =\frac{E_{0}^{x, \tau}\left[e^{\delta_{\tau}-g_{k}\left(\delta_{\tau}\right)}\right]}{E_{0}^{x, \tau}\left[e^{(\rho-1) g_{k}\left(\delta_{\tau}\right)}\right]}
\end{aligned}
$$

For $\delta_{t}=\delta_{0}=0$ we have $g\left(\delta_{t}\right)=g\left(\delta_{0}\right)=g(0)=0$ and the condition requires

$$
1=\int f_{k}(\rho) \lambda_{k}(\rho) d \rho
$$

Because $\lambda_{k}(\rho)$ are decreasing, it follows that if $f_{2}$ stochastically dominates $f_{1}$ then

$$
1=\int f_{1}(\rho) \lambda_{1}(\rho) d \rho>\int f_{2}(\rho) \lambda_{1}(\rho) d \rho
$$

Because $\int f_{2}(\rho) \lambda_{2}(\rho) d \rho=1$, it follows that $\lambda_{2}(\rho)$ has to shift upwards for a range of $\rho$

$$
\lambda_{2}(\rho)>\lambda_{1}(\rho) \text { for some } \rho
$$

Recall now that the functions $g_{k}(\delta)$ have specific properties: They are strictly increasing and concave with $g_{1}(0)=g_{2}(0)=0$ and $g_{k}^{\prime}(\delta) \rightarrow \rho_{H}^{-1}$ for high $\delta$ and viceversa. It follows that $g_{k}(\delta)$ must intersect each other at $\rho=0$. Because $E_{0}^{x, \tau}\left(\delta_{\tau}\right)>0$, and thus $g_{k}\left(\delta_{\tau}\right)>0$ in expectation, we thus must have $g_{2}(\delta)<g_{1}(\delta)$ when $\delta>0$ for $\lambda_{2}(\rho)>\lambda_{1}(\rho)$. Hence, $g_{2}(\delta)>g_{1}(\delta)$ when $\delta<0$. It follows that

$$
g_{2}^{\prime}(\delta)<g_{1}^{\prime}(\delta)
$$

and hence

$$
E_{2}^{*}[\rho \mid \delta]>E_{1}^{*}[\rho \mid \delta]
$$


That is, if $f_{2}(\rho)$ stochastically dominates $f_{1}(\rho)$ then the weighted risk tolerance is higher under $f_{2}$ than under $f_{1}$.

The other claims follow from the formulas for the market price of risk and interest rate, where for the latter, again, we use $\mu / \sigma$ being sufficiently large (e.g. $\mu>0$ and $\sigma \rightarrow 0$ ). Q.E.D.

Proof of Proposition 11: Recall that in Proposition 1 we have

$$
f^{*}\left(\rho \mid \delta_{t}\right)=\frac{f(\rho) \lambda(\rho) e^{\rho g\left(\delta_{t}\right)}}{\int f(\rho) \lambda(\rho) e^{\rho g\left(\delta_{t}\right)} d \rho}
$$

where

$$
\lambda(\rho)=\frac{E_{0}^{x, \tau}\left[e^{\delta_{\tau}-g\left(\delta_{\tau}\right)}\right]}{E_{0}^{x, \tau}\left[e^{(\rho-1) g\left(\delta_{\tau}\right)}\right]}
$$

To understand the impact of a mean-preserving spread (mps), we need to know how the following function is related to $\rho$ :

$$
h\left(\rho ; \delta_{t}\right)=\lambda(\rho) e^{\rho g\left(\delta_{t}\right)}=E_{0}^{x, \tau}\left[e^{\delta_{\tau}-g\left(\delta_{\tau}\right)}\right] \frac{e^{\rho g\left(\delta_{t}\right)}}{E_{0}^{x, \tau}\left[e^{(\rho-1) g\left(\delta_{\tau}\right)}\right]}
$$

We have

$$
\begin{aligned}
h^{\prime}\left(\rho ; \delta_{t}\right) & =E_{0}^{x, \tau}\left[e^{\delta_{\tau}-g\left(\delta_{\tau}\right)}\right]\left[\frac{g\left(\delta_{t}\right) e^{\rho g\left(\delta_{t}\right)}}{E_{0}^{x, \tau}\left[e^{(\rho-1) g\left(\delta_{\tau}\right)}\right]}-\frac{e^{\rho g\left(\delta_{t}\right)} E_{0}^{x, \tau}\left[g\left(\delta_{\tau}\right) e^{(\rho-1) g\left(\delta_{\tau}\right)}\right]}{\left(E_{0}^{x, \tau}\left[e^{(\rho-1) g\left(\delta_{\tau}\right)}\right]\right)^{2}}\right] \\
& =h\left(\rho ; \delta_{t}\right)\left[g\left(\delta_{t}\right)-\frac{E_{0}^{x, \tau}\left[g\left(\delta_{\tau}\right) e^{(\rho-1) g\left(\delta_{\tau}\right)}\right]}{E_{0}^{x, \tau}\left[e^{(\rho-1) g\left(\delta_{\tau}\right)}\right]}\right]
\end{aligned}
$$

If $\mu / \sigma$ is sufficiently high, then $\widetilde{E}_{0}^{x, \tau}\left[g(\mu \tau+\sigma \sqrt{\tau} x) e^{(\rho-1) g(\mu \tau+\sigma \sqrt{\tau} x)}\right]>0$ (note that this is certainly true if $\mu>0$ and $\sigma \rightarrow 0$, given that $g(\delta)>0$ for $\delta>0)$. Given that $g\left(\delta_{t}\right)=0$ for $\delta_{t}=0$ and $g\left(\delta_{t}\right) \rightarrow \infty$ if $\delta_{t} \rightarrow \infty$, it follows that $h^{\prime}\left(\rho ; \delta_{t}\right)<0$ for $\delta_{t}$ sufficiently low, and $h^{\prime}\left(\rho ; \delta_{t}\right)>0$ for $\delta_{t}$ sufficiently high. Let $\bar{\delta}(\rho)$ be such that

$$
g(\bar{\delta}(\rho))=\frac{E_{0}^{x, \tau}\left[g\left(\delta_{\tau}\right) e^{(\rho-1) g\left(\delta_{\tau}\right)}\right]}{E_{0}^{x, \tau}\left[e^{(\rho-1) g\left(\delta_{\tau}\right)}\right]}
$$

Then, $h^{\prime}\left(\rho ; \delta_{t}\right)<0$ for $\delta_{t}<\bar{\delta}(\rho)$ and $h^{\prime}\left(\rho ; \delta_{t}\right)>0$ for $\delta_{t}>\bar{\delta}(\rho)$. Thus, $h^{\prime}\left(\rho ; \delta_{t}\right)<0$ for all $\rho$ if $\delta_{t}<\delta_{\min }=\min _{\rho}(\bar{\delta}(\rho))$ and $h^{\prime}\left(\rho ; \delta_{t}\right)>0$ for all $\rho$ if $\delta_{t}>\delta_{\max }=\max _{\rho}(\bar{\delta}(\rho))$. This argument holds both for the original distribution and the mps distribution. Define $\delta_{\min }^{*}$ and 
$\delta_{\max }^{*}$ the minimum and the maximus across the two distributions. Denote with mps the distributions / variables under the mean-preserving spread. We thus have

$$
f^{*}\left(\rho \mid \delta_{t}\right)=\frac{f(\rho) h\left(\rho ; \delta_{t}\right)}{\int f(\rho) h\left(\rho ; \delta_{t}\right) d \rho} ; f_{m p s}^{*}\left(\rho \mid \delta_{t}\right)=\frac{f_{m p s}(\rho) h_{m p s}\left(\rho ; \delta_{t}\right)}{\int f_{m p s}(\rho) h_{m p s}\left(\rho ; \delta_{t}\right) d \rho}
$$

where $g_{m p s}\left(\delta_{t}\right)$ solves

$$
\delta_{t}=\log \left(\int f_{m p s}(\rho) h_{m p s}\left(\rho ; \delta_{t}\right) d \rho\right)
$$

It follows that if $\mu / \sigma$ is sufficiently high, then when $\delta_{t}<\delta_{\min }^{*}$ and both $h\left(\rho ; \delta_{t}\right)$ and $h_{m p s}\left(\rho ; \delta_{t}\right)$ are decreasing, we have $f_{m p s}^{*}\left(\rho \mid \delta_{t}\right)$ gives relatively more weight to low $\rho$ than $f^{*}\left(\rho \mid \delta_{t}\right)$ does. This implies

$$
E_{m p s}^{*}\left[\rho \mid \delta_{t}\right]<E^{*}\left[\rho \mid \delta_{t}\right] \text { for } \delta_{t}<\delta_{\min }^{*}
$$

Viceversa, if $\delta_{t}>\delta_{\max }^{*}$, then both $h\left(\rho ; \delta_{t}\right)$ and $h_{m p s}\left(\rho ; \delta_{t}\right)$ are increasing and thus $f_{m p s}^{*}\left(\rho \mid \delta_{t}\right)$ gives more weight to high $\rho$ than $f^{*}\left(\rho \mid \delta_{t}\right)$ and we obtain

$$
E_{m p s}^{*}\left[\rho \mid \delta_{t}\right]>E^{*}\left[\rho \mid \delta_{t}\right] \text { for } \delta_{t}>\delta_{\max }^{*}
$$

Q.E.D.

Proof of Proposition 12: Let $s_{k, i}=\bar{s}_{k}\left(\rho_{i}\right) \widetilde{s}_{i}$ where $\operatorname{Cov}\left(\widetilde{s}_{i}, \rho_{i}\right)=0$ and $\mathrm{E}\left[s_{i}\right]=1$, and $\bar{s}_{k}\left(\rho_{i}\right)$ be a monotonic function of $\rho$ such that $E\left[\bar{s}_{k}(\rho)\right]=1$. We can define a new density

$$
f_{k}(\rho)=f(\rho) \bar{s}_{k}(\rho)
$$

This is a density as $\int f_{k}(\rho) d \rho=\int f(\rho) \bar{s}_{k}(\rho) d \rho=E\left[\bar{s}_{k}(\rho)\right]=1$. A model with redistribution is the same as a model with $s(\rho)=1$ (as discussed earlier) but simply with a distribution of $\rho$ 's that is shifted. Consider now the case in which $s_{2}^{\prime}(\rho)>s_{1}^{\prime}(\rho)$. Then $f_{2}(\rho)$ stochastically dominate $f_{1}(\rho)$. The result then follows from corollary 10. Q.E.D.

Proof of Lemma. The lemma follows from the filtering result for discrete Markov processes (see e.g. Chapter 9 in Lyptser and Shyriaev (2001)). Because this filtering result holds for a countable number of states, the limiting distribution $\pi_{t}(\mu)$ and its dynamics can be obtained in the limit as the states are defined as discrete points on the real line and we let the number of states diverge to infinity. Q.E.D.

Proof of Proposition 15: Recall

$$
N_{i t}=\frac{\sigma_{W i t} W_{i t}}{\sigma_{P} P_{t}}
$$


so that

$$
\frac{N_{i t}}{N_{j t}}=\frac{\sigma_{W i t} W_{i t}}{\sigma_{W j t} W_{j t}}
$$

The wealth of each agent is

$$
W_{i t}=p(T-t) s_{i} \lambda\left(\rho_{i}\right) e^{g\left(\delta_{t}\right)} \int E^{x, \tau}\left[e^{\left(\rho_{i}-1\right) g\left(\delta_{t+\tau}\right)} \mid \mu\right] \pi_{t}(\mu) d \mu
$$

Then

$$
\begin{aligned}
d W_{i t}= & o(d t)+p(T-t) s_{i} \lambda\left(\rho_{i}\right) e^{g\left(\delta_{t}\right)}\left[g^{\prime}\left(\delta_{t}\right) \int E^{x, \tau}\left[e^{\left(\rho_{i}-1\right) g\left(\delta_{t+\tau}\right)} \mid \mu\right] \pi_{t}(\mu) d \mu\right. \\
& \left.+\left(\rho_{i}-1\right) \int E^{x, \tau}\left[g^{\prime}\left(\delta_{t+\tau}\right) e^{\left(\rho_{i}-1\right) g\left(\delta_{t+\tau}\right)} \mid \mu\right] \pi_{t}(\mu) d \mu\right] \sigma \\
& +\int E^{x, \tau}\left[e^{\left(\rho_{i}-1\right) g\left(\delta_{t+\tau}\right)} \mid \mu\right] \pi_{t}(\mu)\left(\mu-E_{t}[\mu]\right) d \mu \sigma^{-1} \\
\sigma\left(d W_{i}\right)= & \sigma_{W i t} W_{i t} \\
= & p(T-t) s_{i} \lambda\left(\rho_{i}\right) e^{g\left(\delta_{t}\right)} \int \pi_{t}(\mu)\left\{g^{\prime}\left(\delta_{t}\right) E^{x, \tau}\left[e^{\left(\rho_{i}-1\right) g\left(\delta_{t+\tau}\right)} \mid \mu\right] \sigma\right. \\
& \left.+\left(\rho_{i}-1\right) E^{x, \tau}\left[g^{\prime}\left(\delta_{t+\tau}\right) e^{\left(\rho_{i}-1\right) g\left(\delta_{t+\tau}\right)} \mid \mu\right] \sigma+E^{x, \tau}\left[e^{\left(\rho_{i}-1\right) g\left(\delta_{t+\tau}\right)} \mid \mu\right]\left(\mu-E_{t}[\mu]\right) \sigma^{-1}\right\} d \mu
\end{aligned}
$$

Consider

$h(\mu)=\left[g^{\prime}\left(\delta_{t}\right) \sigma+\left(\mu-E_{t}[\mu]\right) \sigma^{-1}\right] E^{x, \tau}\left[e^{\left(\rho_{i}-1\right) g\left(\delta_{t+\tau}\right)} \mid \mu\right]+\left(\rho_{i}-1\right) E^{x, \tau}\left[g^{\prime}\left(\delta_{t+\tau}\right) e^{\left(\rho_{i}-1\right) g\left(\delta_{t+\tau}\right)} \mid \mu\right] \sigma$

If $h(\mu)$ is convex, then $W_{i t} \sigma_{W i t}$ increases because of MPS in $\pi(\mu)$. Take first derivative

$$
\begin{aligned}
h^{\prime}(\mu)= & \sigma^{-1} E^{x, \tau}\left[e^{\left(\rho_{i}-1\right) g\left(\delta_{t+\tau}\right)} \mid \mu\right] \\
& +\left[g^{\prime}\left(\delta_{t}\right) \sigma+\left(\mu-E_{t}[\mu]\right) \sigma^{-1}\right]\left(\rho_{i}-1\right) E^{x, \tau}\left[g^{\prime}\left(\delta_{t+\tau}\right) \tau e^{\left(\rho_{i}-1\right) g\left(\delta_{t+\tau}\right)} \mid \mu\right] \\
& +\left(\rho_{i}-1\right) E^{x, \tau}\left[\left[g^{\prime \prime}\left(\delta_{t+\tau}\right)+\left(\rho_{i}-1\right) g^{\prime}\left(\delta_{t+\tau}\right)\right] \tau e^{\left(\rho_{i}-1\right) g\left(\delta_{t+\tau}\right)} \mid \mu\right] \sigma
\end{aligned}
$$

Then, the second derivative is

$$
\begin{aligned}
h^{\prime}(\mu)= & \sigma^{-1}\left(\rho_{i}-1\right) E^{x, \tau}\left[g^{\prime}\left(\delta_{t+\tau}\right) \tau e^{\left(\rho_{i}-1\right) g\left(\delta_{t+\tau}\right)} \mid \mu\right] \\
& +\sigma^{-1}\left(\rho_{i}-1\right) E^{x, \tau}\left[g^{\prime}\left(\delta_{t+\tau}\right) \tau e^{\left(\rho_{i}-1\right) g\left(\delta_{t+\tau}\right)} \mid \mu\right] \\
& +\left[g^{\prime}\left(\delta_{t}\right) \sigma+\left(\mu-E_{t}[\mu]\right) \sigma^{-1}\right]\left(\rho_{i}-1\right) E^{x, \tau}\left[\left[g^{\prime \prime}\left(\delta_{t+\tau}\right)+\left(\rho_{i}-1\right) g^{\prime}\left(\delta_{t+\tau}\right)\right] \tau^{2} e^{\left(\rho_{i}-1\right) g\left(\delta_{t+\tau}\right)} \mid \mu\right] \\
& +\left(\rho_{i}-1\right) E^{x, \tau}\left[\left[g^{\prime \prime \prime}\left(\delta_{t+\tau}\right)+\left(\rho_{i}-1\right) g^{\prime \prime}\left(\delta_{t+\tau}\right)\right] \tau^{2} e^{\left(\rho_{i}-1\right) g\left(\delta_{t+\tau}\right)} \mid \mu\right] \sigma \\
& +\left(\rho_{i}-1\right) E^{x, \tau}\left[\left[g^{\prime \prime}\left(\delta_{t+\tau}\right)+\left(\rho_{i}-1\right) g^{\prime}\left(\delta_{t+\tau}\right)\right]\left(\left(\rho_{i}-1\right) g^{\prime}\left(\delta_{t+\tau}\right)\right) \tau^{2} e^{\left(\rho_{i}-1\right) g\left(\delta_{t+\tau}\right)} \mid \mu\right] \sigma
\end{aligned}
$$

or

$$
\begin{aligned}
h "(\mu)= & 2 \sigma^{-1}\left(\rho_{i}-1\right) E^{x, \tau}\left[g^{\prime}\left(\delta_{t+\tau}\right) \tau e^{\left(\rho_{i}-1\right) g\left(\delta_{t+\tau}\right)} \mid \mu\right] \\
& +\left[g^{\prime}\left(\delta_{t}\right) \sigma+\left(\mu-E_{t}[\mu]\right) \sigma^{-1}\right]\left(\rho_{i}-1\right) E^{x, \tau}\left[\left[g^{\prime \prime}\left(\delta_{t+\tau}\right)+\left(\rho_{i}-1\right) g^{\prime}\left(\delta_{t+\tau}\right)\right] \tau^{2} e^{\left(\rho_{i}-1\right) g\left(\delta_{t+\tau}\right)} \mid \mu\right] \\
& +\left(\rho_{i}-1\right) E^{x, \tau}\left[\left[g^{\prime \prime \prime}\left(\delta_{t+\tau}\right)+\left(\rho_{i}-1\right) g^{\prime \prime}\left(\delta_{t+\tau}\right)\right] \tau^{2} e^{\left(\rho_{i}-1\right) g\left(\delta_{t+\tau}\right)} \mid \mu\right] \sigma \\
& +\left(\rho_{i}-1\right)^{2} E^{x, \tau}\left[\left[g^{\prime \prime}\left(\delta_{t+\tau}\right)+\left(\rho_{i}-1\right) g^{\prime}\left(\delta_{t+\tau}\right)\right] g^{\prime}\left(\delta_{t+\tau}\right) \tau^{2} e^{\left(\rho_{i}-1\right) g\left(\delta_{t+\tau}\right)} \mid \mu\right] \sigma
\end{aligned}
$$


We consider the local curvature of $h(\mu)$ around the mean $\widehat{\mu}_{t}$. In this case $\mu-E[\mu]$ is small (for given $\sigma$ ). When $\sigma$ is small, moreover, only the first term is large, as all the others converge to zero as $\sigma$ converges to zero. Therefore, for $\sigma$ small, the local curvature of $h(\mu)$ is

$$
h^{\prime \prime}(\mu) \approx 2 \sigma^{-1}\left(\rho_{i}-1\right) E^{x, \tau}\left[g^{\prime}\left(\delta_{t+\tau}\right) \tau e^{\left(\rho_{i}-1\right) g\left(\delta_{t+\tau}\right)} \mid \mu\right]
$$

which is positive for $\rho_{i}>1$ and negative for $\rho_{i}<1$. Therefore, for $\sigma$ small and $\mu$ around $\widehat{\mu}_{t}$, we should expect that a mean preserving spread increases $\sigma(d W)$ for $\rho_{i}>1$ and decrease it for $\rho_{i}<1$. The result follows. Q.E.D. 